\title{
Magnetic fields in spiral galaxies
}

\author{
Rainer Beck ${ }^{1}$
}

Received: 30 June 2015 / Published online: 29 December 2015

(C) The Author(s) 2015

\begin{abstract}
Radio synchrotron emission, its polarization and Faraday rotation of the polarization angle are powerful tools to study the strength and structure of magnetic fields in galaxies. Unpolarized synchrotron emission traces isotropic turbulent fields which are strongest in spiral arms and bars $(20-30 \mu \mathrm{G})$ and in central starburst regions (50-100 $\mu \mathrm{G})$. Such fields are dynamically important; they affect gas flows and drive gas inflows in central regions. Polarized emission traces ordered fields, which can be regular or anisotropic turbulent, where the latter originates from isotropic turbulent fields by the action of compression or shear. The strongest ordered fields $(10-15 \mu \mathrm{G})$ are generally found in interarm regions. In galaxies with strong density waves, ordered fields are also observed at the inner edges of spiral arms. Ordered fields with spiral patterns exist in grand-design, barred and flocculent galaxies and in central regions. Ordered fields in interacting galaxies have asymmetric distributions and are a tracer of past interactions between galaxies or with the intergalactic medium.-Faraday rotation measures of the diffuse polarized radio emission from galaxy disks reveal large-scale spiral patterns that can be described by the superposition of azimuthal modes; these are signatures of regular fields generated by mean-field dynamos. "Magnetic arms" between gaseous spiral arms may also be products of dynamo action, but need a stable spiral pattern to develop. Helically twisted field loops winding around spiral arms were found in two galaxies so far. Large-scale field reversals, like the one found in the Milky Way, could not yet be detected in external galaxies. In radio halos around edgeon galaxies, ordered magnetic fields with X-shaped patterns are observed. The origin and evolution of cosmic magnetic fields, in particular their first occurrence in young
\end{abstract}

Rainer Beck

rbeck@mpifr-bonn.mpg.de

1 Auf dem Hügel 69, 53121 Bonn, Germany 
galaxies and their dynamical importance during galaxy evolution, will be studied with forthcoming radio telescopes like the Square Kilometre Array.

Keywords Polarization - Magnetic fields - Dynamo - Galaxies: magnetic fields · Galaxies: spiral · Galaxies: halos · Radio continuum: galaxies

\section{The role of magnetic fields in spiral galaxies}

Whether and how magnetic fields influence the formation and evolution of galaxies is still unknown. Most numerical models of the interstellar medium in galaxies ignored magnetic fields. Magneto-hydrodynamical (MHD) models of evolving galaxies are computationally challenging and require various simplifications (Wang and Abel 2009; Pakmor and Springel 2013; Pakmor et al. 2014).

Much more is known about magnetic fields in the interstellar medium (ISM) of nearby galaxies and the Milky Way, thanks to modern radio telescopes, the construction of sensitive and wide-band receiving systems and the development of new methods of observation and data analysis in the radio range. Major progress was also achieved in numerical modelling of the magnetized ISM (e.g. Avillez and Breitschwerdt 2005; Gissinger et al. 2009; Hill et al. 2012; Gressel et al. 2013; Gent et al. 2013; Machida et al. 2013), using a variety of codes and assumptions. Not surprisingly, the results differ and are not always consistent with observations, which calls for further efforts on both sides.

A widespread argument to neglect galactic magnetic fields in the past was based on assuming field strengths of a few $\mu \mathrm{G}$ taken from Faraday rotation data in our Milky Way, which trace only a small component of the total field. The dynamical importance of galactic fields, with typical strengths of $15 \mu \mathrm{G}$, is now widely appreciated in many fields of astrophysics. Magnetic and cosmic-ray pressures control the overall star-formation rate (Birnboim et al. 2015). Magnetic fields are important for the gas dynamics of molecular clouds (Planck Collaboration et al. 2015a). With stronger fields fewer cloud cores are formed but with larger masses (Vázquez-Semadeni et al. 2005; Price and Bate 2008). Magnetic fields control the density and propagation of cosmic rays. Together with cosmic rays they can provide the pressure to drive fast outflows of hot gas, in particular in galaxies with high star-formation rates in the early Universe (Hanasz et al. 2013). Cosmic rays propagating along the field lines in galaxy halos may heat the warm ionized gas and explain the optical line ratios (Wiener et al. 2013). Outflows from starburst galaxies may have magnetized the intergalactic medium (Bertone et al. 2006).

The detection of ultrahigh-energy cosmic rays (UHECRs) reaching the Earth with the AUGER observatory and the possibly anisotropic distribution of their arrival directions (Aab et al. 2015) calls for a proper model of particle propagation. As UHECR particles are deflected by large-scale regular fields and scattered by turbulent fields, the structure and the extent of the fields in the disk and halo of the Milky Way need to be known, but are hard to measure from our inside location. The view onto external spiral galaxies can help. 


\section{Origin of galactic magnetic fields}

Building up magnetic fields in galaxies is a three-stage process: (1) seeding, (2) amplifying, (3) ordering and sustaining.

1. Seed fields can be "primordial", generated in the early Universe (Durrer and Neronov 2013), or they may originate from later epochs, e.g. during cosmological structure formation by the Weibel instability (Lazar et al. 2009), from plasma fluctuations in protogalaxies (Schlickeiser 2012; Schlickeiser and Felten 2013), from injection by the first stars (Bisnovatyi-Kogan et al. 1973) or jets generated by the first black holes (Rees 2005) or from the Biermann mechanism in the first supernova remnants (Hanayama et al. 2005).

Indication for a weak all-pervasive intergalactic seed field as a relic from the early Universe comes from the non-detection of GeV $\gamma$-ray emission with the FERMI satellite from blazars, which were observed at TeV energies with the HESS observatory. The secondary particles generated by the $\mathrm{TeV}$ photons may be deflected by intergalactic fields of at least $10^{-16} \mathrm{G}$ strength and a high-volume filling factor (Dolag et al. 2011). However, fluctuations in the intergalactic plasma may also disperse the $\gamma$-ray emission (Broderick et al. 2012) (see also the discussion in Durrer and Neronov 2013). Analysis of the CMB power spectra data from PLANCK gave an upper limit of about $5 \mathrm{nG}$ on a (comoving) scale of $1 \mathrm{Mpc}$ (Planck Collaboration et al. 2015b).

2. An efficient source of field amplification is turbulence in the gas driven by supernova explosions (Ferrière 1996) or by spiral shocks (Kim et al. 2006), called the small-scale dynamo. In protogalaxies this mechanism can amplify weak seed fields to several $\mu \mathrm{G}$ strength (the energy level of turbulence) within less than $10^{8}$ year (Kulsrud et al. 1997; Schleicher et al. 2010; Beck et al. 2012a; Rieder and Teyssier 2015). The resulting field is turbulent.

3. The final and most time-consuming stage is the ordering of the turbulent field. Numerical models of evolving galaxies show fast field amplification and ordering with help of differential rotation (Wang and Abel 2009; Kotarba et al. 2009), possibly supported by the magneto-rotational instability (MRI) (Gressel et al. 2013; Pakmor and Springel 2013). In these models the ordered magnetic field forms spiral arm segments, but it has frequent reversals in azimuthal and radial directions and hence is anisotropic turbulent (Sect. 3.1).

The most promising mechanism to sustain magnetic fields and generate large-scale regular fields from turbulent fields in the interstellar medium of galaxies is the $\alpha-\Omega$ dynamo (Beck et al. 1996). It is based on differential rotation $(\Omega)$, expanding gas flows, driven by supernova explosions (Ferrière 1996; Gressel et al. 2013), or cosmic rays (Hanasz et al. 2009), carrying magnetic fields that are twisted by the Coriolis force ( $\alpha$-effect), and on magnetic diffusivity $(\eta)$ driven by turbulence. The "meanfield" approximation of the $\alpha-\Omega$ dynamo equation allows analytical solutions by mathematically separating the large-scale and small-scale parts of the velocity field and the magnetic field. The "mean-field" dynamo equation for the regular field B

$$
\partial \mathbf{B} / \partial t=\nabla \times(\mathbf{v} \times \mathbf{B})+\nabla \times \alpha \mathbf{B}+\eta \nabla^{2} \mathbf{B}
$$


describes the field amplification by the induction term $\nabla \times(\mathbf{v} \times \mathbf{B})$, where $\mathbf{v}$ is the largescale velocity, the gain term $\nabla \times \alpha \mathbf{B}$ and the loss term $\eta \nabla^{2} \mathbf{B}$. MHD modelling with high spatial resolution showed that the scale separation of the mean-field approximation is reasonable (Gent et al. 2013).

Solutions of the mean-field dynamo equation are described by modes $m$ with different azimuthal symmetries in the disk plane (axisymmetric, bisymmetric, quadrisymmetric, etc.) and two different vertical symmetries (even parity or odd parity) perpendicular to the disk plane. Several modes can be excited in the same object, where lower modes have shorter growth times and larger amplitudes. In flat, rotating objects like galaxy disks, the strongest mode is composed of a toroidal field of axisymmetric spiral shape within the disk $(m=0)$, without sign reversals across the equatorial plane and a weaker poloidal field of even symmetry. Azimuthal dynamo modes can be identified observationally from the patterns of polarization angles and Faraday rotation measures of the diffuse polarized emission from galaxy disks (see Sect. 4.7).

Mean-field models of the $\alpha-\Omega$ dynamo in galaxy disks predict that turbulent fields are transformed into large-scale regular fields within a few $10^{9}$ year (Beck et al. 1994; Arshakian et al. 2009; Rodrigues et al. 2015). Field reversals from the early phases may survive until today (Moss et al. 2012). Global numerical models of galaxies (Gissinger et al. 2009; Hanasz et al. 2009; Kulpa-Dybeł et al. 2011; Siejkowski et al. 2014) confirmed the basic results of the mean-field models.

The twisted field loops needed for the operation of the $\alpha-\Omega$ dynamo generate large-scale helicity. ${ }^{1}$ As total helicity in a system is a conserved quantity (Brandenburg and Subramanian 2005), small-scale fields with opposite helicity are generated which quench dynamo action, unless they are removed from the system (Shukurov et al. 2006). Hence, moderately fast outflows are essential for effective $\alpha-\Omega$ dynamo action, but strong outflows can suppress dynamos. This leaves a certain range of outflow velocities for optimal dynamo action (Rodrigues et al. 2015). As outflows are strongest above spiral arms, the $\alpha-\Omega$ dynamo is expected to be more efficient between the spiral arms (Chamandy et al. 2015).

The total magnetic field $B_{\text {tot }}$ can be presented as the quadratic sum of the regular field $B_{\text {reg }}$ generated by the $\alpha-\Omega$ dynamo and the component $B_{\text {turb }}$ generated by the small-scale dynamo. Tangling of $B_{\text {reg }}$ can produce another component, $B_{\tan }$. $B_{\tan }$ and $B_{\text {turb }}$ should be comparable, because both components are driven by the same turbulent gas motions. On the solar surface, the small-scale dynamo seems to be weak (Stenflo 2012), but may still operate in the solar interior. In contrast to the Sun, stars and planets, the regime of dynamo action is fully accessible in galaxies. The existence of the small-scale dynamo is of fundamental importance for the evolution of magnetic fields in galaxies. Without a small-scale dynamo the seed fields in protogalaxies would be much weaker and the generation of large-scale regular fields would take much longer. Turbulent and tangled fields can be distinguished with (future) high-resolution observations.

\footnotetext{
${ }^{1}$ Magnetic helicity $H$ is defined as $H=A \cdot(\nabla \times A)$, where $A$ is the magnetic vector potential.
} 


\section{Observational tools}

Magnetic fields need illumination to be detectable. Polarized emission at optical, infrared, submillimeter and radio wavelengths holds the clue to measure magnetic fields in galaxies. Huge progress has been achieved in the past decade.

The various methods to measure magnetic fields are briefly discussed in the following. The basic concepts are presented in more detail in Klein and Fletcher (2015).

\subsection{Basic magnetic field components}

Table 1 summarizes the different field components and the methods used to observe them.

The total magnetic field is separated into a regular and a turbulent component. A regular field has a well-defined direction within the telescope beam, while a turbulent field frequently reverses its direction within the telescope beam. In other words, the coherence scale of regular fields is much larger than the turbulent scale. Turbulent fields can be isotropic turbulent (i.e. the same dispersion in all three spatial dimensions) or anisotropic turbulent (i.e. different dispersions). Anisotropic turbulent fields originate from isotropic turbulent fields by the action of compressing or shearing gas flows.

The power spectra of turbulent fields are generally assumed to be of Kolmogorov type, with the largest scale given by the scale driving turbulence (e.g. supernova

Table 1 Magnetic field components and methods to observe them

\begin{tabular}{|c|c|c|}
\hline Field component & Notation & Observational signatures \\
\hline $\begin{array}{l}\text { Total field perpendicular to the } \\
\text { line of sight }\end{array}$ & $B_{\text {tot }, \perp}^{2}=B_{\text {turb }, \perp}^{2}+B_{\text {reg }, \perp}^{2}$ & Total synchrotron intensity \\
\hline $\begin{array}{l}\text { Turbulent or tangled field } \\
\text { perpendicular to the line of } \\
\text { sight }\end{array}$ & $B_{\text {turb }, \perp}^{2}=B_{\text {iso }, \perp}^{2}+B_{\text {aniso }, \perp}^{2}$ & $\begin{array}{l}\text { Total synchrotron emission, } \\
\text { partly polarized }\end{array}$ \\
\hline $\begin{array}{l}\text { Isotropic turbulent or tangled } \\
\text { field perpendicular to the line } \\
\text { of sight }\end{array}$ & $B_{\text {iso }, \perp}\left(=\sqrt{2 / 3} B_{\text {iso }}\right)$ & $\begin{array}{l}\text { Unpolarized synchr. intensity, } \\
\text { beam depolarization, Faraday } \\
\text { depolarization }\end{array}$ \\
\hline $\begin{array}{l}\text { Isotropic turbulent or tangled } \\
\text { field along the line of sight }\end{array}$ & $B_{\text {iso, } \|}\left(=\sqrt{1 / 3} B_{\text {iso }}\right)$ & Faraday depolarization \\
\hline $\begin{array}{l}\text { Ordered field perpendicular to the } \\
\text { line of sight }\end{array}$ & $B_{\text {ord }, \perp}^{2}=B_{\text {aniso }, \perp}^{2}+B_{\text {reg }, \perp}^{2}$ & $\begin{array}{l}\text { Intensity and vectors of radio, } \\
\text { optical, IR and submm pol. }\end{array}$ \\
\hline $\begin{array}{l}\text { Anisotropic turbulent or tangled } \\
\text { field perpendicular to the line } \\
\text { of sight }\end{array}$ & $B_{\text {aniso, }, \perp}$ & $\begin{array}{l}\text { Intensity and vectors of radio, } \\
\text { optical, IR and submm pol., } \\
\text { Faraday depolarization }\end{array}$ \\
\hline $\begin{array}{l}\text { Regular field perpendicular to the } \\
\text { line of sight }\end{array}$ & $B_{\text {reg }, \perp}$ & $\begin{array}{c}\text { Intensity and vectors of radio, } \\
\text { optical, IR and submm pol., } \\
\text { Goldreich-Kylafis effect }\end{array}$ \\
\hline $\begin{array}{l}\text { Regular field along the line of } \\
\text { sight }\end{array}$ & $B_{\text {reg, } \|}$ & $\begin{array}{l}\text { Faraday rotation + depol., } \\
\text { longitudinal Zeeman effect }\end{array}$ \\
\hline
\end{tabular}

The symbols $\perp$ and $\|$ indicate field components perpendicular and parallel to the line of sight, respectively 
remnants in galaxies) and the smallest scale given by magnetic diffusivity. The power spectra of regular fields generated by the mean-field dynamo show a broad maximum over a range of scales which develops with time (Moss et al. 2012).

Present-day radio polarization observations with limited spatial resolution cannot distinguish turbulent fields generated by turbulent gas flows from tangled fields generated from regular fields by small-scale gas motions; the components of both types of fields in the sky plane give rise to unpolarized synchrotron emission.

Anisotropic turbulent, anisotropic tangled and regular field components contribute to the ordered field, observable in polarized synchrotron emission. Polarization angles are ambiguous by $180^{\circ}$ so that polarized emission is not sensitive to field reversals. Faraday rotation and the longitudinal Zeeman effect are sensitive to the field direction and hence trace regular fields.

\subsection{Optical, infrared and submm polarization}

Elongated dust grains can be oriented with their major axis perpendicular to the interstellar magnetic field lines by paramagnetic alignment (the Davis-Greenstein effect) or, more efficiently, by radiative torque alignment (Hoang and Lazarian 2008, 2014). When particles on the line of sight to a star are oriented with their major axis perpendicular to the line of sight (and the field is oriented in the same plane), the different levels of extinction along the major and the minor axis lead to polarization of the starlight, with the E-vectors pointing parallel to the component of the interstellar magnetic field perpendicular to the line of sight (Fig. 1). The interpre-

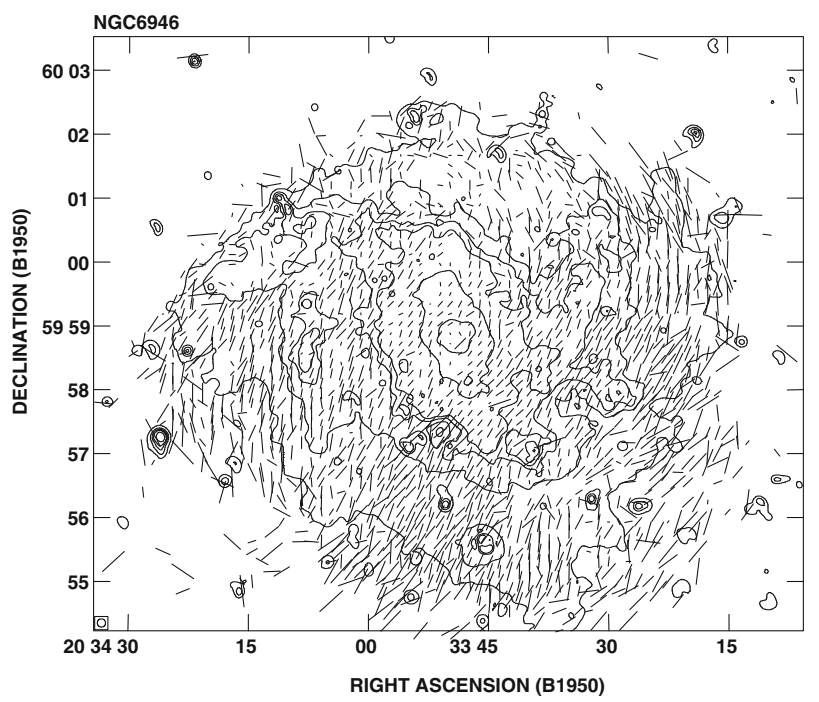

Fig. 1 Optical emission (contours) and polarization E-vectors of NGC 6946, observed with the polarimeter of the Landessternwarte Heidelberg at the MPIA Calar Alto 1-m telescope. The optical E-vectors are along the spiral arms in the eastern and western regions (compare with the radio polarization B-vectors in Fig. 13). Polarization due to light scattering is probably occurring in the southern part (from Fendt et al. 1998) 
tation of optical and near-infrared polarization observations of individual stars or diffuse starlight is based on this mechanism. Extinction is most efficient for grains of sizes similar to the wavelength. These small particles are only aligned in the medium between molecular clouds, not in the dense clouds themselves (Cho and Lazarian 2005).

The detailed physics of the alignment is complicated and not fully understood. The degree of polarization p generated in a volume element along the line of sight of extent $L$ is not only proportional to $\left(B_{\mathrm{tot}, \perp}^{2} L\right)$, but also dependent on the magnetic properties, density and temperature of the grains (Ellis and Axon 1978).

Starlight polarization yields the orientation of large-scale magnetic fields in the Milky Way (Sect. 5), and in the galaxies M 51 (Scarrott et al. 1987), NGC 6946 (Fig. 1) and the Small Magellanic Cloud (Lobo Gomes et al. 2015). The major shortcoming when applying this method to extended sources like gas clouds or galaxies is that light can also be polarized by scattering, a contamination that is unrelated to magnetic fields and difficult to subtract.

At far-infrared and submillimeter wavelengths, elongated dust grains emit linearly polarized waves, without contributions by polarized scattered light. The B-vector is parallel to the magnetic field. The field structure can be mapped in gas clouds of the Milky Way, e.g. in the massive star formation site W51 (Tang et al. 2009) and in galaxies, e.g. in the halo of the starburst galaxy M 82 (Greaves et al. 2000).

Magnetic force (total field strength $B$ ) and kinetic force by turbulent gas motions (turbulent velocity $v_{\text {turb }}$ and density $\rho$ ) are in competition in the interstellar medium. In a strong field the field lines are straight and the dispersion of polarization angles $\sigma$ is small. According to Chandrasekhar and Fermi (1953), the total field strength $B_{\text {tot, } \perp}$ in the sky plane can be estimated from

$$
B_{\mathrm{tot}, \perp} \simeq\left(\frac{4}{3} \pi \rho\right)^{1 / 2} v_{\text {turb }} / \sigma
$$

Application to starlight polarization in the Milky Way yielded field strengths of about $7 \mu \mathrm{G}$, in agreement with other methods (Sect. 5). The Chandrasekhar-Fermi method was improved by correcting for the observational errors in polarization angle and signal integration (Hildebrand et al. 2009; Houde et al. 2009, 2011). It can also be applied to radio polarization angles, like in M 51 (Houde et al. 2013).

\subsection{Zeeman effect}

The Zeeman effect is the most direct method of remote sensing of magnetic fields. In the presence of a magnetic field $B_{\|}$along the line of sight, a spectral line is split into two circularly polarized components of opposite sign (longitudinal Zeeman effect). The frequency shift is $2.8 \mathrm{MHz} / \mathrm{Gauss}$ for the $\mathrm{H} \mathrm{I}$ line and larger for molecular lines like $\mathrm{OH}, \mathrm{CN}$ or $\mathrm{H}_{2} \mathrm{O}$ (Heiles and Crutcher 2005). The Zeeman effect is sensitive to regular fields, but turbulent fields can also be measured from the dispersion of the circularly polarized signal at a large number of locations on the source (Watson et al. 2001). The interpretation of Zeeman splitting is hampered by its weakness, requiring high line 
intensities and careful correction of instrumental polarization. Most results have been obtained for the H I line that traces clouds of diffuse (warm) gas in the Milky Way (Crutcher et al. 2010) and for the $\mathrm{OH}$ line for starburst galaxies (Robishaw et al. 2008).

Magnetic fields perpendicular to the line of sight generate two shifted lines in addition to the main unshifted line, all linearly polarized (transversal Zeeman effect). These lines cannot be resolved for observations in the Milky Way and external galaxies and no net polarization is observed under symmetric conditions. Detection of linearly polarized lines becomes possible for unequal populations of the different sublevels, a gradient in optical depth or velocity or an anisotropic velocity field (Goldreich and Kylafis 1981). Depending on the line ratios, the orientation of linear polarization can be parallel or perpendicular to the magnetic field orientation. This Goldreich-Kylafis effect was detected in molecular clouds, star-forming regions, outflows of young stellar systems and supernova remnants of the Milky Way. It was also observed in the ISM of the galaxy M 33 (Li and Henning 2011), where it is consistent with the field orientations along the spiral arms as measured by polarized radio continuum emission (Tabatabaei et al. 2008). The $\pm 90^{\circ}$ ambiguity needs to be solved with help of additional continuum polarization data.

\subsection{Total radio synchrotron emission and the equipartition assumption}

Radio continuum emission from galaxies is a mixture of thermal and nonthermal (synchrotron) components. The average thermal fractions are typically 10 and $25 \%$ at 20 and $6 \mathrm{~cm}$ wavelengths, respectively. At long wavelengths thermal emission is negligible, but thermal absorption may reduce the synchrotron intensity in starforming regions. A first-order separation of the thermal and nonthermal components can be performed by assuming a uniform spectral index of $\alpha=0.8-1.0$ for the nonthermal intensity $\left(I \propto v^{-\alpha}\right)$ and using the spectral index of the thermal emission in an optically thin plasma of $\alpha=0.1$. However, synchrotron intensity depends on energy and age of cosmic-ray electrons (CREs) propagating from the spiral arms and losing energy by synchrotron radiation and inverse Compton scattering with photons so that the synchrotron spectrum is expected to be flatter (smaller $\alpha$ ) in spiral arms than in interarm regions, outer disk and halo. The advanced method to separate the two components introduced by Tabatabaei et al. (2007) uses an extinction-corrected $H \alpha$ image as a template for the thermal radio emission and was first applied to the galaxy M 33. Simplified separation methods use an $H \alpha$ image (Heesen et al. 2014) or a combination of $H \alpha$ and $24 \mu \mathrm{m}$ infrared images as a thermal template that is subtracted from the total emission (Basu and Roy 2013).

The intensity of total synchrotron emission (see examples in Sect. 4) is a measure of the number density of cosmic-ray electrons (CREs) in the relevant energy range and of the strength of the total magnetic field component $B_{\text {tot, } \perp}$ perpendicular to the line of sight (i.e. in the sky plane). Synchrotron emission at a frequency $v$ is emitted by a range of CREs with average energy $E$ (in $\mathrm{GeV}$ ) in a field of strength $B_{\text {tot, } \perp \text { (in }}$ $\mu \mathrm{G})$ :

$$
v \simeq 16 \mathrm{MHz} E^{2} B_{\mathrm{tot}, \perp} .
$$


Equipartition is expected between the energy densities of the total cosmic rays (dominated by protons) and the total magnetic field. The cosmic-ray energy density is determined by integrating over their energy spectrum. This allows us to calculate the total magnetic field strength from the synchrotron intensity (Beck and Krause 2005; Arbutina et al. 2012). The synchrotron intensity $I_{\text {syn }}$ scales with the total magnetic field strength as $I_{\text {syn }} \propto B_{\text {tot, } \perp}^{3+\alpha}$. Vice versa, $B_{\text {tot }, \perp \text { scales as }}$

$$
B_{\text {tot }, \perp} \propto\left(I_{\text {syn }}\left(K_{0}+1\right) / L\right)^{1 /(3+\alpha)},
$$

where $\alpha$ is the synchrotron spectral index $\left(I_{\text {syn }} \propto v^{-\alpha}\right)$ and $L$ is the effective pathlength through the source. $K_{0}$ is the ratio of number densities of CR protons and electrons. $K_{0} \simeq 100$ is a reasonable assumption in the star-forming regions of the disk (Bell 1978). For an electron-positron plasma, e.g. in jets of radio galaxies, $K_{0}=0$. The input parameters, especially the pathlength $L$ and the ratio $K_{0}$ are not well constrained. Changing one of these values by a factor of, e.g. 2 changes the field strength only weakly by $2^{1 /(3+\alpha)} \simeq 1.2$.

Issues with the equipartition estimate are

1. Equation 4 can be applied only for steep radio spectra with $\alpha>0.5$. For flatter spectra, the integration over the energy spectrum of the cosmic rays has to be restricted to a limited energy interval.

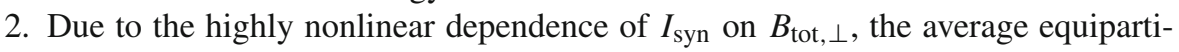
tion value $B_{\mathrm{tot}, \perp}$ derived from synchrotron intensity is biased towards high field strengths and hence is an overestimate if $B_{\text {tot }}$ varies along the line of sight or across the telescope beam (Beck et al. 2003). For $\alpha=1$ and the equipartition case, the overestimation factor $g$ of the total field is (see Appendix A in Stepanov et al. 2014):

$$
g=\left(\left\langle B_{\mathrm{tot}, \perp}^{4}\right\rangle\right)^{1 / 4} /\left\langle B_{\mathrm{tot}, \perp}\right\rangle=\left(1+\frac{8}{3} Q^{2}+\frac{8}{9} Q^{4}\right)^{1 / 4},
$$

where \langle\rangle indicates the averages along the line of sight and the beam, and $Q=\left(\left\langle\delta B_{\text {tot }, \perp}^{2}\right\rangle\right)^{1 / 2} /\left\langle B_{\text {tot }, \perp}\right\rangle$ is the amplitude of the field fluctuations relative to the mean field. For strong fluctuations of $Q=1$, the overestimation factor is 1.46 .

3. If energy losses of CREs are significant, especially in starburst regions or massive spiral arms, the equipartition values are lower limits and are underestimated. The ratio $K$ increases because energy losses of ageing CREs are much more severe than those of cosmic-ray protons. Using the standard value $K_{0}$ underestimates the total magnetic field by a factor of $\left(K / K_{0}\right)^{1 /(3+\alpha)}$ in such regions (Beck and Krause 2005). The same applies to the outer disk and halo of a galaxy, where the emitting CREs propagated far away from the places of origin and energy losses are significant.

4. In dense gas, e.g. in starburst regions, secondary positrons and electrons may be responsible for most of the radio emission via pion decay. Notably, the ratio of protons to secondary electrons is also about 100 for typical radio wavelengths (Lacki and Beck 2013). 
5. Energy equipartition needs time to develop and hence is not valid on small scales in time and space. A correlation analysis of radio continuum images from the Milky Way and the nearby galaxy M 33 by Stepanov et al. (2014) showed that equipartition does not hold on scales smaller than about $1 \mathrm{kpc}$, which is understandable in view of the typical propagation length of CREs away from their sources (Sect. 4.3). The equipartition field strength is underestimated in regions around the CRE sources and overestimated in regions far away from the sources.

Arguments for the validity of equipartition on large $(\mathrm{kpc})$ scales come from the joint analysis of radio continuum and $\gamma$-ray data, allowing an independent determination of magnetic field strengths, e.g. in the Milky Way (Strong et al. 2000), the Large Magellanic Cloud (LMC) (Mao et al. 2012) and in M 82 (Yoast-Hull et al. 2013) ${ }^{2}$. Furthermore, the estimates of strengths of large-scale ordered fields derived from Faraday rotation data are similar to those from equipartition in several galaxies (see Table 2 in Van Eck et al. 2015).

\subsection{Polarized radio synchrotron emission}

Linearly polarized synchrotron emission (see examples in Sect. 4) emerges from CREs in ordered fields in the sky plane. As polarization angles are ambiguous by $180^{\circ}$, they cannot distinguish regular fields, defined to have a constant direction within the telescope beam, from anisotropic turbulent or tangled fields. Unpolarized synchrotron emission indicates isotropic turbulent or tangled fields that have random directions in 3D and have been amplified by turbulent gas flows (see Sect. 3.1).

The intrinsic degree of linear polarization $p_{0}$ of synchrotron emission in a perfectly regular field is

$$
p_{0}=(1+\alpha) /\left(\frac{5}{3}+\alpha\right)
$$

where $\alpha$ is the spectral index of the synchrotron emission. In spiral galaxies, typical values are $\alpha=0.8-1.0$ so that $p_{0}=73-75 \%$.

The observed degree of polarization is smaller than $p_{0}$ due to the contribution from unpolarized thermal emission, wavelength-dependent Faraday depolarization (Sect. 3.7) and wavelength-independent "beam depolarization" that is discussed in the following.

Turbulent magnetic fields in galaxies preserve their direction over a coherence scale that depends on the properties of turbulence. ${ }^{3}$ In the case of isotropic turbulent fields with a constant coherence length $d$, the observable degree of synchrotron polarization is [see Sokoloff et al. (1998)for a detailed discussion]:

\footnotetext{
2 But probably not in NGC 253 (Yoast-Hull et al. 2014).

3 The degrees of polarization expected from compressed or sheared fields are discussed in the Appendix of Beck et al. (2005).
} 


$$
p=p_{0} N^{-1 / 2}
$$

where $N \simeq D^{2} L f / d^{3}$ is the number of synchrotron-emitting turbulent cells with diameter $d$ and filling factor $f$ within the volume defined by the telescope beam with the linear size $D$ at the galaxy's distance and pathlength $L$ through the source. Equation (7) is valid only at short radio wavelengths, where Faraday depolarization is small.

Typical degrees of synchrotron polarization observed in nearby galaxies between 3 and $6 \mathrm{~cm}$ wavelengths with typical spatial resolutions of $D \simeq 500 \mathrm{pc}$ are $1-5 \%$ in spiral arms (where isotropic turbulent fields dominate), e.g. in NGC 6946 (Beck 2007) and in M 33 (Tabatabaei et al. 2008). With $L \simeq 1000 \mathrm{pc}$ and $f \simeq 0.5$ for the turbulent field coupled to the warm diffuse gas, we get $d \simeq 50 \mathrm{pc}$, consistent with estimates from Faraday depolarization at long wavelengths (Eq. 15).

If the medium is pervaded by an isotropic turbulent field $B_{\text {iso }}$ plus an ordered field $B_{\text {ord }}$ (regular and/or anisotropic turbulent) that has a constant orientation in the volume observed by the telescope beam, the wavelength-independent polarization (for the case of equipartition between the energy densities of magnetic field and cosmic rays) amounts to (Sokoloff et al. 1998):

$$
p=p_{0}\left(1+3.5 q^{2}\right) /\left(1+4.5 q^{2}+2.5 q^{4}\right)
$$

where $q=B_{\text {iso }, \perp} / B_{\text {ord }, \perp}$ and $B_{\text {iso }, \perp}=\sqrt{2 / 3} B_{\text {iso }}$.

\subsection{Faraday rotation and Faraday synthesis}

The polarization plane is rotated in a magnetized thermal plasma by Faraday rotation. As the rotation angle is sensitive to the sign of the field direction, only regular fields give rise to Faraday rotation, while the Faraday rotation contributions from turbulent fields largely cancel along the line of sight. Measurements of Faraday rotation from multiwavelength observations (see examples in Sect. 4.7) yield the strength and direction of the average regular field component along the line of sight. If Faraday rotation is low (in galaxies typically at wavelengths shorter than a few centimetres), the B-vector of polarized emission gives the intrinsic field orientation in the sky plane so that the magnetic pattern can be mapped directly (Sect. 4.5).

The Faraday rotation measure $R M$ is defined as the slope of the observed variation of the polarization rotation angle $\chi$ with the square of wavelength $\lambda$ :

$$
\chi=\chi_{0}+R M \lambda^{2}
$$

where $\chi_{0}$ is the intrinsic polarization angle. $\lambda$ is measured in metres and $\mathrm{RM}$ in $\mathrm{rad} \mathrm{m}^{-2}$. $\mathrm{RM}$ is constant if $\chi$ is a linear function of $\lambda^{2}$, e.g. if one or more Faraday-rotating regions are located in front of the emitting region (Faraday screen).

A nonlinear variation of $\chi$ with $\lambda^{2}$ and hence a variation of RM with $\lambda^{2}$ occurs in case of the following: 
1. emission and rotation in the same region where the distribution of electrons or regular magnetic field strength along the line of sight is not symmetric, or field reversals occur or the field is helical (Sokoloff et al. 1998),

2. Faraday depolarization (Sect. 3.7),

3. several distinct emitting and rotating regions with different contributions to Faraday rotation are located within the beam or along the line of sight or within the source (internal structure).

In these cases, RM needs to be replaced by the Faraday depth $\Phi$ (Burn 1966):

$$
\chi=\chi_{0}+\Phi \lambda^{2}, \quad \text { where } \Phi=0.808 \int_{\text {source }}^{\text {observer }} B_{\mathrm{reg}, \|} n_{\mathrm{e}} \mathrm{d} l
$$

where $\Phi$ is measured in $\operatorname{radm}^{-2}$, the line-of-sight magnetic field $B_{\mathrm{reg}, \|}$ in $\mu \mathrm{G}$, the thermal electron density $n_{\mathrm{e}}$ in $\mathrm{cm}^{-3}$ and the line of sight $l$ in pc.

To measure the contribution of various sources to $\Phi$, wide-band, multi-channel spectro-polarimetric radio data are needed. These are Fourier-transformed by a software tool, called RM synthesis (Brentjens and de Bruyn 2005; Heald 2015) or Faraday synthesis (following the terminology proposed by Sun et al. 2015), to obtain the Faraday spectrum $F(\Phi)$ (previously called Faraday dispersion function) at each position of the radio image. $F(\Phi)$ shows the intensity of polarized emission and its polarization angle as a function of $\Phi$ (Fig. 2).

If the medium has a relatively simple structure, Faraday spectra at several positions can reveal the 3D structure of the magnetized interstellar medium (Faraday tomography). For example, regular fields, field reversals and turbulent fields can be recognized from their signatures in Faraday spectra (Bell et al. 2011; Frick et al. 2011; Beck et al. 2012b). Helical fields can also imprint characteristic features in the Faraday spectrum (Brandenburg and Stepanov 2014; Horellou and Fletcher 2014). Realistic galaxies have complicated Faraday spectra, as revealed from simplified galaxy models (Ideguchi et al. 2014).

Crucial to successful application of Faraday synthesis is that the observations cover a wide range in $\lambda^{2}$, giving high resolution in Faraday spectra. Even for a wide frequency coverage, two components with a difference in intrinsic polarization angle of about $90^{\circ}$ cannot be properly recovered by Faraday synthesis so that model fitting to the data in Stokes Q and U is needed (Farnsworth et al. 2011).

A grid of RM measurements of polarized background sources is another powerful tool to measure magnetic field patterns in galaxies (Stepanov et al. 2008; Mao et al. 2012). At least ten RM values from sources behind a galaxy's disk are needed to recognize a simple large-scale field pattern if the Galactic foreground contribution is constant and the background sources have no intrinsic contributions to RM.

Measuring the strength of the regular field from RM needs additional information about the thermal electron density, e.g. from the arrival times of pulsar signals that are delayed in a cloud of ionized gas proportional to the dispersion measure $\mathrm{DM}=\int n_{\mathrm{e}} \mathrm{d} l$. 


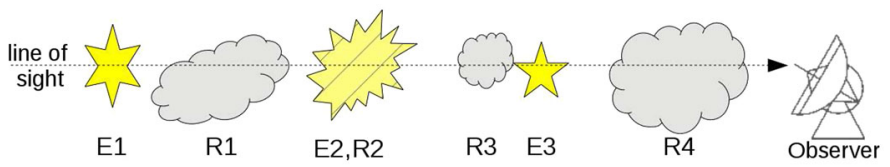

(a)

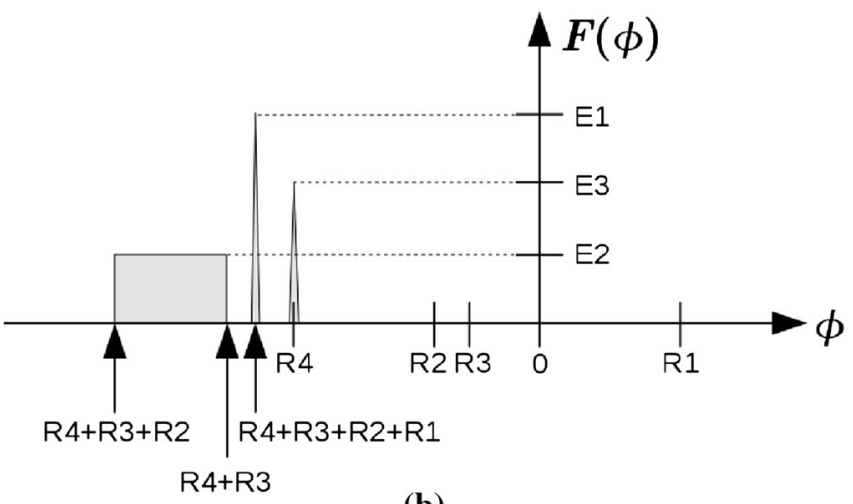

(b)

Fig. 2 Example for a set of different polarization-emitting and rotating components along the line of sight and the resulting Faraday spectrum $F(\Phi) . E 1$ and $E 3$ are point sources, $E 2, R 2$ is an extended emitting and rotating region. $R 1, R 3$ and $R 4$ are Faraday screens that are not emitting. Faraday depolarization effects are neglected here for simplicity (from Gießübel et al. 2013). a Sketch of different components along the line of sight. Some of them are emitting $(E i)$, are Faraday-rotating $(R i)$, or both. b Resulting Faraday spectrum $F(\Phi)$, where $\Phi$ is the Faraday depth, of the components depicted in a

The ratio

$$
\mathrm{RM} / \mathrm{DM}=0.808\left\langle B_{\mathrm{reg}, \|} n_{\mathrm{e}}\right\rangle /\left\langle n_{\mathrm{e}}\right\rangle
$$

allows us to compute the field strength $B_{\text {reg, } \|}$ (in $\mu \mathrm{G}$ ) if the fluctuations in field strength and in electron density are uncorrelated. The value for $B_{\text {reg, } \|}$ derived from pulsar data is an underestimate if small-scale fluctuations in field strength and in electron density are anticorrelated, as expected for local pressure equilibrium, while it is an overestimate if the fluctations are correlated, as expected for compression by shock fronts or turbulence (Beck et al. 2003).

\subsection{Faraday depolarization}

In a single region containing CREs, thermal electrons and purely regular magnetic fields, wavelength-dependent Faraday depolarization occurs because the polarization planes of waves from the far side of the emitting layer are more rotated than those from the near side. This effect is called differential Faraday rotation. For one single layer with a symmetric distribution of thermal electron density and field strength along the line of sight the degree of polarization is reduced to (Burn 1966; Sokoloff et al. 1998): 


$$
p=p_{0}\left|\sin \left(2 \mathrm{RM} \lambda^{2}\right) /\left(2 \mathrm{RM} \lambda^{2}\right)\right|,
$$

where RM is the observed rotation measure from the source, which is half of the Faraday depth $\Phi$ through the whole layer. $p$ varies periodically with the square of wavelength.

Applying Faraday synthesis (Sect. 3.6) to polarization data of a region that is emitting and Faraday-rotating reveals an extended component in the Faraday spectrum (Fig. 2). However, regions broader than $\Phi_{\max } \simeq \pi / \lambda_{\min }^{2}$ cannot be recovered, where $\lambda_{\min }$ is the minimum wavelength of the observations (Brentjens and de Bruyn 2005); only two "horns" remain visible at the edges of the structure in the Faraday spectrum $F(\Phi)$ (Beck et al. 2012b). This problem is similar to the missing short baselines in synthesis imaging.

For multiple emitting + rotating layers, the Faraday spectrum contains several extended components and Eq. (12) is no longer applicable. Shneider et al. (2014) extended Burn's model to a multi-layer (e.g. galaxy disk + halo) medium.

Faraday rotation in helical fields has a completely different behaviour and may lead to an increase of the degree of polarization with increasing wavelength ("repolarization") in certain wavelength ranges (Sokoloff et al. 1998; Brandenburg and Stepanov 2014; Horellou and Fletcher 2014).

Turbulent fields also cause wavelength-dependent depolarization, called Faraday dispersion (Sokoloff et al. 1998; Arshakian and Beck 2011). For an emitting and Faraday-rotating region (internal dispersion) the degree of polarization is reduced to

$$
p=p_{0}(1-\exp (-S)) / S,
$$

where $S=2 \sigma_{\mathrm{RM}}^{2} \lambda^{4}$. $\sigma_{\mathrm{RM}}$ is the dispersion in rotation measure and depends on the turbulent field strength along the line of sight, the turbulence scale, the thermal electron density, and the pathlength through the medium.

Depolarization by external dispersion occurs in a turbulent Faraday-rotating (but not emitting) medium in the foreground if the diameter of the telescope beam at the distance of the screen is larger than the turbulence scale:

$$
p=\exp (-S)
$$

An important effect of Faraday dispersion is that the interstellar medium becomes "Faraday thick" for polarized radio emission beyond a certain wavelength, depending on $\sigma_{\mathrm{RM}}$, and only a front layer remains visible in polarized intensity. Typical values for galaxy disks are $\sigma_{\mathrm{RM}}=20-100 \mathrm{rad} \mathrm{m}^{-2}$, while star-forming regions can have dispersions of $\sigma_{\mathrm{RM}} \simeq 1000 \mathrm{radm}^{-2}$ (Arshakian and Beck 2011). The maximum in the spectrum of polarized intensity falls into the wavelength range 2$20 \mathrm{~cm}$.

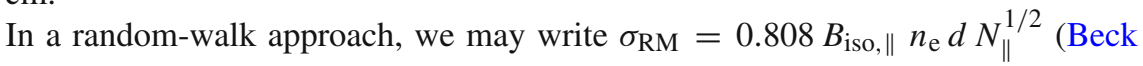
2007), where $B_{\text {iso, } \|}=\sqrt{1 / 3} B_{\text {iso }}$ is the strength of the isotropic turbulent field, $n_{\mathrm{e}}$ is the electron density within a cell of size $d$, and $N_{\|}=L f / d$ is the number of cells along the line of sight $L$ with a volume filling factor $f$. The average electron density along the line of sight is $\left\langle n_{\mathrm{e}}\right\rangle=n_{\mathrm{e}} / f$ so that we get 


$$
\sigma_{\mathrm{RM}}=0.808 B_{\text {iso }, \|}\left\langle n_{\mathrm{e}}\right\rangle \sqrt{L d / f} .
$$

The average value for galaxy disks of $\sigma_{\mathrm{RM}} \simeq 50 \mathrm{rad} \mathrm{m}^{-2}$ is consistent with typical values for the warm diffuse ISM of $\left\langle n_{\mathrm{e}}\right\rangle \simeq 0.03 \mathrm{~cm}^{-3}$ and $f \simeq 0.5$ (Fig. $10 \mathrm{in}$ Berkhuijsen et al. 2006), $B_{\text {iso }} \simeq 10 \mu \mathrm{G}, L \simeq 1000 \mathrm{pc}$, and $d \simeq 50 \mathrm{pc}$.

The dispersion $\sigma_{\mathrm{RM}}$ leads to a "Faraday forest" of $N$ components in the Faraday spectrum. If $N$ is not large, the components are possibly resolvable with very high Faraday resolution and hence a wide $\lambda^{2}$ span of the observations (Beck et al. 2012b; Bernet et al. 2012).

\section{Results}

\subsection{Magnetic field strengths}

Strength is the fundamental quantity to estimate the dynamical importance of magnetic fields. The equipartition assumption (Sect. 3.4) is the only presently applicable method to measure the total field strength. Zeeman measurements will become possible in spiral galaxies with future radio telescopes, but will trace fields on small scales only.

The average equipartition strength (Eq. (4)) of total fields (corrected for inclination) for a sample of 74 spiral galaxies is $B_{\mathrm{tot}}=9 \pm 2 \mu \mathrm{G}$ (Niklas 1995). The average strength of 21 bright galaxies observed between 2000 and 2010 is $B_{\text {tot }}=17 \pm 3 \mu \mathrm{G}$ (Fletcher 2010). Gas-rich spiral galaxies with high star-formation rates, like M 51 (Fig. 5), M 83 (Fig. 7) and NGC 6946 (Fig. 13), have total field strengths of 20-30 $\mu \mathrm{G}$ in their spiral arms. The strongest total fields of $50-100 \mu \mathrm{G}$ are found in starburst galaxies like M 82 (Adebahr et al. 2013), in the "Antennae" NGC 4038/9 (Chyży and Beck 2004), in nuclear starburst regions like in NGC 253 (Heesen et al. 2011a), and in barred galaxies (Beck et al. 2005). In such galaxies, energy losses of CREs can be strong so that the equipartition values are underestimates (Sect. 3.4). Radio-faint galaxies like M 31 (Fig. 11) and M 33 have weaker total magnetic fields (about $6 \mu \mathrm{G}$ ). The similarity to the values derived for our Milky Way with various methods (Sect. 5) gives confidence that the equipartition assumption is valid.

Field strengths of $0.5-18 \mathrm{mG}$ were detected in starburst galaxies by the Zeeman effect in the $\mathrm{OH}$ megamaser emission line at $18 \mathrm{~cm}$ wavelength (Robishaw et al. 2008). These values refer to highly compressed gas clouds and are not typical of the diffuse interstellar medium.

The strength of ordered fields in the sky plane as observed by polarized synchrotron emission varies strongly between galaxies, from 10 to $15 \mu \mathrm{G}$ in M 51 and the magnetic arms of NGC 6946 (Fig. 13) to about $5 \mu \mathrm{G}$ in the star-forming ring of M 31 (Fig. 11). The average strength of the ordered fields of 21 bright galaxies observed since 2000 is $B_{\text {ord }, \perp}=5 \mu \mathrm{G}$ with a standard deviation of $3 \mu \mathrm{G}$ and an average ratio $B_{\text {ord }, \perp} / B_{\text {tot }}$ of 0.3 (Fletcher 2010).

The only deep observation of an Sa galaxy, M 104, with a prominent dust ring, revealed ordered, partly regular magnetic fields (Krause et al. 2006). Spiral galaxies of type $\mathrm{S} 0$ and elliptical galaxies without an active nucleus have very little star formation and hence hardly produce CREs that could emit synchrotron emission. Irregular 


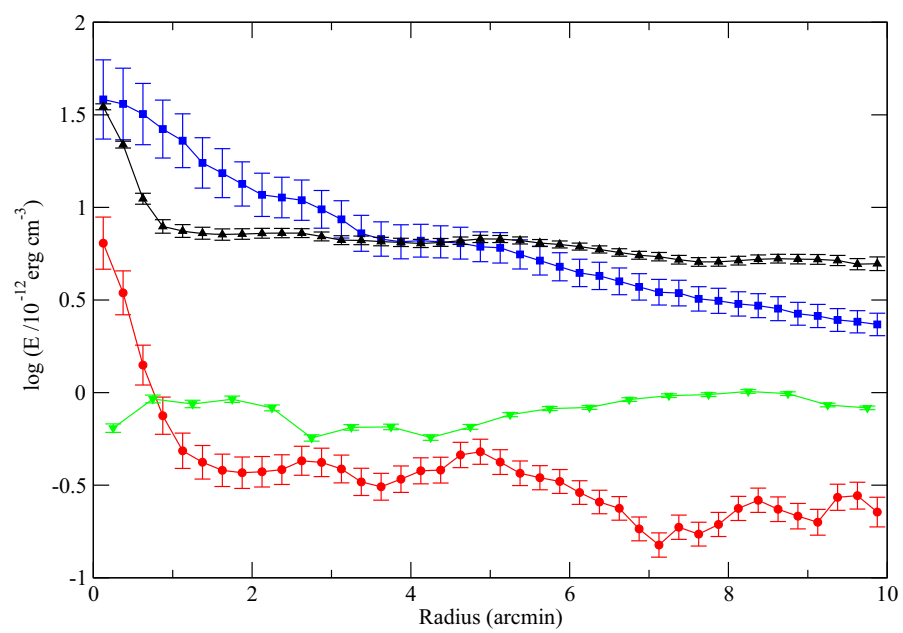

Fig. 3 Radial variation of the energy densities in IC 342, determined from observations of synchrotron and thermal radio continuum and the $\mathrm{CO}$ and $\mathrm{H}$ I line emissions: for the total magnetic field $B_{\text {tot }}^{2} / 8 \pi$ (black triangles), identical to that for the total cosmic rays, for the ordered magnetic field $B_{\mathrm{ord}}^{2} / 8 \pi$ (green triangles), for the turbulent motion of the neutral gas $E_{\text {turb }}=0.5 \rho_{n} \mathrm{v}_{\text {turb }}{ }^{2}$ (blue squares), where $\mathrm{v}_{\mathrm{turb}}=7 \mathrm{~km} / \mathrm{s}$, and for the thermal energy of the warm ionized gas $E_{\mathrm{th}}=1.5\left\langle n_{\mathrm{e}}\right\rangle k T_{\mathrm{e}}$ (red circles), where $T_{\mathrm{e}}=10^{4} \mathrm{~K}$. The error bars include only errors due to rms noise in the images from which the energy densities are derived. No systematic errors are included, e.g. those imposed by a radial variation of thermal gas temperature, filling factor or turbulent gas velocity, nor errors due to deviations from the equipartition assumption (from Beck 2015)

starburst galaxies show only spots of ordered fields (Heesen et al. 2011b). No ordered fields could be detected so far in dwarf irregular galaxies (Chyży et al. 2011).

\subsection{Magnetic energy densities}

The relative importance of various competing forces in the interstellar medium can be estimated by comparing the corresponding energy densities. The mean energy densities of the total (mostly turbulent) magnetic field and the total cosmic rays, averaged in rings of about $1 \mathrm{kpc}$ width, are $\simeq 10^{-11} \mathrm{erg} \mathrm{cm}^{-3}$ in NGC 6946 (Beck 2007), M 63, M 83, NGC 4736 (Basu and Roy 2013) and IC 342 (Fig. 3), and $\simeq 10^{-12} \mathrm{erg} \mathrm{cm}^{-3}$ in M 33 (Tabatabaei et al. 2008). In all these galaxies the average magnetic energy density is similar to the average kinetic energy density of the turbulent motions of the neutral gas across the star-forming disk (assuming a constant turbulent velocity of $10 \mathrm{~km} \mathrm{~s}^{-1}$ ) and about ten times larger than that of the warm ionized gas (but still 500-1000 times smaller than the energy density of the general rotation of the neutral gas). This result is similar to that derived for the Milky Way (Cox 2005). The ISM is a low- $\beta$ plasma (where $\beta$ is the ratio of thermal to magnetic energy densities). Magnetic fields are dynamically important.

According to Fig. 3, $E_{\text {turb }} / E_{\text {th }} \gg$ 1, i.e. turbulence is supersonic. Supersonic turbulence leads to shocks and hence dissipation of kinetic energy into heat, until turbulence 
becomes transonic and the system reaches an equilibrium state. The observation of supersonic turbulence may indicate that a quasi-steady state is never reached. However, observational bias effects need to be considered. The energy density of hot gas in the ISM, neglected in Fig. 3, is similar or somewhat larger than that of warm gas, depending on its volume filling factor (Ferrière 2001) so that its inclusion would not change the above result significantly. Another bias is the assumption of a constant gas temperature $T_{\mathrm{e}}$. If cosmic rays propagate via the streaming instability (Sect. 4.3), they may heat the gas and increase $T_{\mathrm{e}}$ in the outer disk (Wiener et al. 2013).

Another important message from Fig. 3 (and similarly from the other galaxies studied so far) is that the magnetic energy density decreases radially more slowly than the kinetic energy density and dominates in the outer galaxy disk, i.e. the Alfvén velocity $\mathrm{v}_{\mathrm{A}} \propto B_{\mathrm{tot}} / \sqrt{\rho}$ is larger than the turbulent velocity and increases with radius, which may cause magnetic effects on the rotation curve of the gas (Elstner et al. 2014). Note that the ratio of magnetic to kinetic energy may be even higher in the outer galaxy disk than shown in Fig. 3, because the equipartition field strengths are underestimates due to energy losses of the CREs (Sect. 3.4). Furthermore, the turbulent velocity tends to decrease with radius (Tamburro et al. 2009), which would also enhance the ratio of magnetic to kinetic energy.

The following are the possible reasons why equipartition between magnetic and kinetic energy density does not hold within galaxies:

1. energy or pressure balance is valid between the magnetic field and the sum of pressures related to all gas components (kinetic and thermal) (Basu and Roy 2013);

2. the efficiency of the small-scale dynamo increases from the inner to the outer galaxy;

3. the magneto-rotational instability (MRI) leads to super-equipartition fields (Fig. 7 in Gressel et al. 2013);

4. the equipartition field strengths are underestimated in the inner galaxy and overestimated in the outer galaxy due to CRE propagation (see Sect. 3.4), thus flattening the profile of the magnetic energy density.

Scenario (4) is supported by the analysis of radio and IR images (Berkhuijsen et al. 2013) and the cross-correlation based on wavelet transforms that breaks down below a certain scale that can be interpreted as the propagation length of CREs (Tabatabaei et al. 2013a). CRE propagation in IC 342 seems to be exceptionally fast (Sect. 4.3).

The energy density of the ordered magnetic field (Fig. 3) even increases radially. This field may be assumed to be mostly regular so that we can invoke the $\alpha-\Omega$ dynamo that also operates in the outer disk of galaxies (Mikhailov et al. 2014). Although the star-formation activity is low in the outer disk, the magneto-rotational instability (MRI) may serve as the source of turbulence required for $\alpha-\Omega$ dynamo action (Sellwood and Balbus 1999; Gressel et al. 2013). Alternatively, regular fields generated in the inner disk could be transported outwards by the joint action of a dynamo and turbulent diffusivity (Moss et al. 1998). 


\subsection{Cosmic-ray propagation}

The measurable extent $L_{\text {syn }}$ of synchrotron disks or halos (see Sect. 4.13) gives us information about the extent of the total magnetic field and the propagation of CREs. Under energy equipartition conditions, the radial exponential scale length of the total field in a disk of mildly inclined galaxies (or the vertical exponential scale height in a halo of almost edge-on galaxies) is $L_{\mathrm{B}} \geq(3+\alpha) L_{\text {syn }}$, where $\alpha \simeq 0.9$ is the synchrotron spectral index. The scale length $L_{\mathrm{CR}}$ (or scale height) of the total cosmic rays is half of this value. These are lower limits because the CREs lose their energy with distance from the star-forming disk so that the equipartition assumption yields too small values for the field strength (Sect. 3.4). The scale lengths of synchrotron disks of typically $L_{\text {syn }} \simeq 3-5 \mathrm{kpc}$ at $20 \mathrm{~cm}$ wavelength (Beck 2007; Basu and Roy 2013; Mulcahy et al. 2014) yield $L_{\mathrm{B}} \simeq 12-19 \mathrm{kpc}$ and $L_{\mathrm{CR}} \simeq 6-9.5 \mathrm{kpc}$. The nearby galaxy IC 342 (Fig. 6) reveals an exceptionally large synchrotron scale length of about $16 \mathrm{kpc}$ (Beck 2015).

The radial scale length and its frequency dependence give us a general idea about the speed and type of CRE propagation. At wavelengths of $\geq 1 \mathrm{~m}$, synchrotron and inverse Compton losses are weaker than at shorter wavelengths so that CREs can diffuse further outwards from their locations of origin. This is clearly seen in M 31 and M 33 (Berkhuijsen et al. 2013). The LOFAR image of the galaxy M 51 at $2 \mathrm{~m}$ wavelength reveals a steepening of the radial distribution of synchrotron emission, located at about $10 \mathrm{kpc}$ radius, beyond the sharp decrease in the star-formation rate, indicating a propagation length of a few kpc (Mulcahy et al. 2014). The frequency dependence of the scale length favours diffusive propagation in the cases of M 51 and several other galaxies (Basu and Roy 2013; Berkhuijsen et al. 2013; Mulcahy et al. 2014) and fast convective transport in IC 342 (Beck 2015).

The galactic fields may extend further out into intergalactic space than visible in radio synchrotron images. A large radial scale length means that magnetic fields may affect the global rotation of the gas in the outer parts of spiral galaxies, possibly explaining some fraction of the flattening of the rotation curves (Battaner and Florido 2007; Elstner et al. 2014).

\subsection{Relations between gas, star formation and magnetic fields}

Radio continuum and infrared emissions from galaxies are closely correlated.

First, a global correlation exists between the galaxy-integrated luminosity of the total radio continuum emission at around $20 \mathrm{~cm}$ wavelengths (frequencies of around $1 \mathrm{GHz}$ ), which is mostly of synchrotron origin and the infrared (IR) luminosity of starforming galaxies. This is one of the tightest correlations known in astronomy. The correlation extends over five orders of magnitude (Bell 2003), is slightly nonlinear in log-log scale (Bell 2003; Pierini et al. 2003) with an exponent of $1.09 \pm 0.05$ (Basu et al. 2015) and is valid in starburst galaxies to redshifts of at least four (Seymour et al. 2008), without evidence for evolution with redshift up to 1.2 (Basu et al. 2015). As the correlation between radio thermal and IR luminosity should be strictly linear, the exponent of the nonthermal (synchrotron)-IR correlation is steeper than 1.1. A 
breakdown of synchrotron emission and of its correlation with IR is expected beyond a critical redshift when inverse Compton loss of the CREs dominates synchrotron loss; this critical redshift will give us information about the field evolution in young galaxies (Schleicher and Beck 2013).

In galaxies with strong magnetic fields, e.g. in starburst galaxies, the CREs may lose their energy by synchrotron loss within the galaxy disk ("lepton calorimeter model", Völk 1989), where the correlation is linear and due to the joint generation of CREs and dust-heating UV photons in star-forming regions, independent of the magnetic field. In Milky Way-type galaxies, magnetic fields are crucial to understand the correlation. Niklas and Beck (1997) proposed an "equipartition model", where total (mostly turbulent) magnetic fields, cosmic rays, gas density and star formation are related and predicted a nonlinear synchrotron-IR correlation with an exponent of about 1.3 , in good agreement with observations. The more detailed analysis by Lacki et al. (2010) identified multiple feedback mechanisms that are not yet fully understood.

Second, the total radio and IR intensities within galaxies are also highly correlated, but with a smaller exponent, due to CRE propagation (Berkhuijsen et al. 2013). CRE propagation is also responsible for the flattening of the profile of cosmic-ray energy density in Fig. 3. The exponent of the correlation was found to be different in the central region, spiral arms and interarm regions in several nearby galaxies (Dumas et al. 2011; Basu et al. 2012), and it also differs between galaxies due to differences in the diffusion length of CREs (Berkhuijsen et al. 2013; Tabatabaei et al. 2013a).

The magnetic field and its structure play an important role in understanding the correlation (Tabatabaei et al. 2013a, b). The synchrotron-IR correlation can be presented as a correlation between total field strength $B_{\text {tot }}$ and star-formation rate surface density $\Sigma_{\text {SFR }}$ with an exponent of $0.18 \pm 0.01$ within NGC 4254 (Chyży 2008), $0.14 \pm 0.01$ within NGC 6946 (Fig. 4) and $0.30 \pm 0.02$ (globally and locally) for a sample of 17 spiral galaxies (Heesen et al. 2014). A very similar exponent is expected from theoretical considerations (Schleicher and Beck 2013).

The Kennicutt-Schmidt law $\Sigma_{\text {SFR }} \propto \Sigma_{\text {gas }}^{N}$ is valid within galaxies, with $N \simeq 1.0$ for the molecular gas and $N \simeq 1.5$ for the atomic gas (Bigiel et al. 2008). ${ }^{4}$ Then the above relation $B_{\text {tot }} \propto \Sigma_{\text {SFR }}^{0.30}$ corresponds to $B_{\text {tot }} \propto \Sigma_{\text {gas }}^{0.30}$ if molecular gas dominates, e.g. in the inner regions of most spiral galaxies, and $B_{\text {tot }} \propto \Sigma_{\text {gas }} 0.45$ in the outer regions that are dominated by atomic gas.

Turbulent fields in spiral arms are probably generated by turbulent gas motions related to star formation activity (Sect. 2). The small-scale dynamo predicts equipartition between magnetic and kinetic energy densities $\left(B_{\text {tot }} \propto \rho^{0.5}\right.$, assuming $\mathrm{v}_{\text {turb }}=$ const), which is supported by the observed average values of a galaxy sample (Niklas and Beck 1997). However, within a galaxy the ratio between these energy densities decreases (Fig. 3), which is consistent with an exponent of $<0.5$ found from the $\Sigma_{\text {SFR }}$ data (see above). Possible reasons why equipartition between magnetic and kinetic energy density holds on average between galaxies, but not within galaxies, were discussed in Sect. 4.2.

\footnotetext{
4 The nonlinearity of the Kennicutt-Schmidt law can be interpreted as a dependence of the star-formation efficiency (SFE) on the stellar mass surface density (Shi et al. 2011). Interestingly, effects of the magnetic field on the SFE have not been investigated so far.
} 


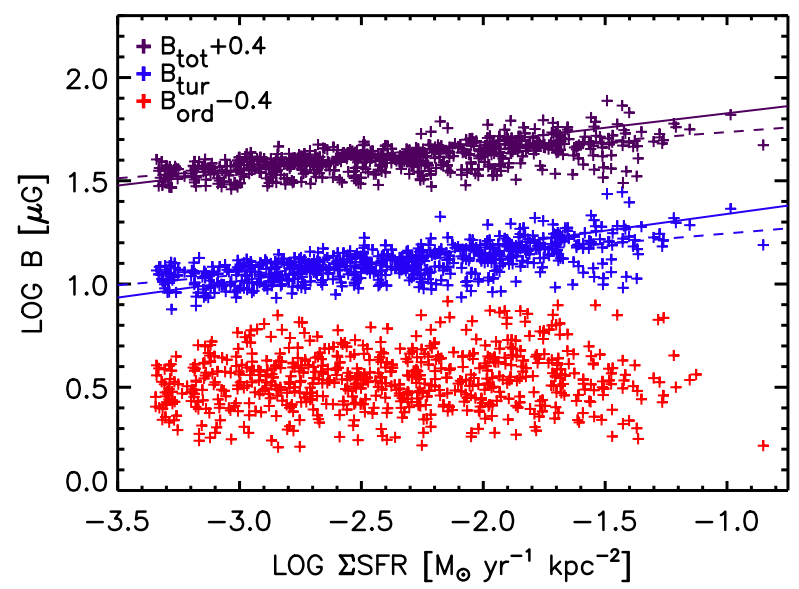

Fig. 4 Correlation between the strength of the total, turbulent and ordered fields and the star-formation rate per area (determined from the $24 \mu \mathrm{m}$ infrared intensities) within the galaxy NGC 6946. The points for $B_{\text {tot }}$ and $B_{\text {ord }}$ are shifted by +0.4 and -0.4 , respectively, to fit into the same figure. The solid lines are bisector fits with slopes of $0.14 \pm 0.01$ for $B_{\text {tot }}$ and $0.16 \pm 0.01$ for $B_{\text {turb }}$, the dashed lines ordinary least-squares fits with slopes $0.089 \pm 0.004$ and $0.100 \pm 0.005$ (from Tabatabaei et al. 2013b and priv. comm.)

The correlation between the radio continuum luminosity and the molecular gas (traced by its $\mathrm{CO}$ line emission) is similarly tight as the radio-IR correlation and is also nonlinear, with a slope of $1.31 \pm 0.09$ (Liu and Gao 2010). The correlation between the synchrotron radio continuum and $\mathrm{CO}$ intensities, observed with $60 \mathrm{pc}$ resolution within the spiral galaxy M 51 (Schinnerer et al. 2013), appears to be tighter than the radio-IR correlation and may be the fundamental one. Much of the molecular gas in the spiral arms of M 51 is not directly associated with ongoing massive star formation, implying that star formation cannot be the sole cause for the radio-CO relation. Either the synchrotron emission emerges from secondary CREs that are produced in the interaction of cosmic-ray protons with the dense molecular material (Murgia et al. 2005), or the synchrotron emission is from primary CREs in strong magnetic fields coupled to the dense gas (Niklas and Beck 1997), in connection with fast diffusion of CREs along the spiral arms. High-resolution observations of nearby galaxies (e.g. with SKA and ALMA) can provide the necessary information on the coupling between magnetic fields and gas on small scales, to test which of the proposed origins for the correlation is indeed true.

In contrast to the turbulent field, the ordered field is either uncorrelated with the starformation rate (Fig. 4) or anticorrelated in interarm regions, where the star-formation rate is low (Frick et al. 2001).

\subsection{Spiral fields}

The most striking result from the observation of ordered (regular and/or anisotropic) fields with help of polarized synchrotron emission is that spiral patterns are found in almost every spiral galaxy (Beck and Wielebinski 2013), in galaxies with a star- 


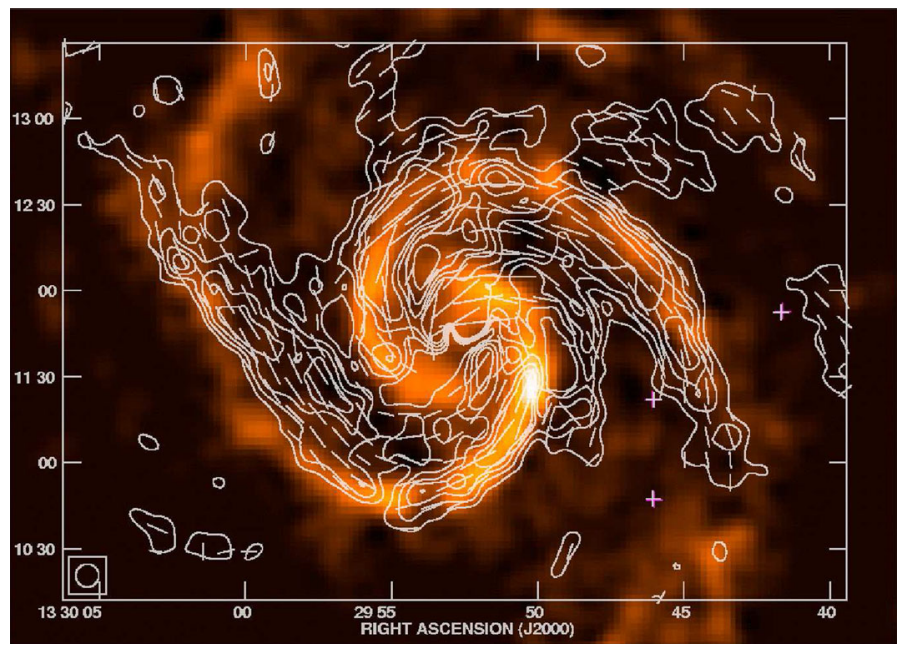

Fig. 5 Polarized radio emission (contours) and B-vectors of M 51, combined from observations at $6 \mathrm{~cm}$ wavelength with the VLA and Effelsberg telescopes at $8^{\prime \prime}$ resolution (from Fletcher et al. 2011). The background colour image shows the $\mathrm{CO}$ line emission from molecular gas (from Helfer et al. 2003)

forming ring (Chyży and Buta 2008), in flocculent galaxies without gaseous spiral arms (Soida et al. 2002), in central regions of galaxies (Fig. 17) and in circum-nuclear gas rings of barred galaxies (Fig. 16).

In galaxies with strong density waves like M 51 (Fig. 5) and M 83 (Fig. 7) enhanced ordered fields occur at the inner edges of the inner optical arms where the cold molecular gas is densest (Patrikeev et al. 2006). These are probably anisotropic turbulent fields generated by the compression of the density wave. An analysis of dispersions of the radio polarization angles at $6 \mathrm{~cm}$ wavelength in $M 51$ shows that the ratio of the correlation lengths parallel and perpendicular to the local ordered magnetic field is about two (Houde et al. 2013).

In galaxies without strong density waves the ordered fields are not coinciding with the gaseous or the optical spiral arms. The typical degree of radio polarization at small wavelengths is only a few per cent within the spiral arms, hence the field in the spiral arms must be mostly tangled or randomly oriented within the telescope beam on scales of a few $10 \mathrm{pc}$ (Eq. 7).

Ordered fields are strongest $(10-15 \mu \mathrm{G})$ in the regions between the spiral arms and oriented parallel to the adjacent spiral arms. In some galaxies they form magnetic arms, like in NGC 6946 (Fig. 13), with exceptionally high degrees of polarization up to $50 \%$. These are probably generated by the $\alpha-\Omega$ dynamo (Sect. 4.9).

The ordered magnetic field in the galaxy IC 342 reveals several spiral polarization arms of different origins (Fig. 6). In contrast to NGC 6946, there is only a rudimentary magnetic arm in an interarm region in the north-west, probably because of weaker dynamo action in IC 342 (Sect. 4.9). A narrow polarization arm of about $300 \mathrm{pc}$ width, displaced inwards with respect to the inner arm east of the central region by about $200 \mathrm{pc}$, indicates that magnetic fields are compressed by a density wave, like in M 51. A broad polarization arm of $300-500 \mathrm{pc}$ width around the northern 


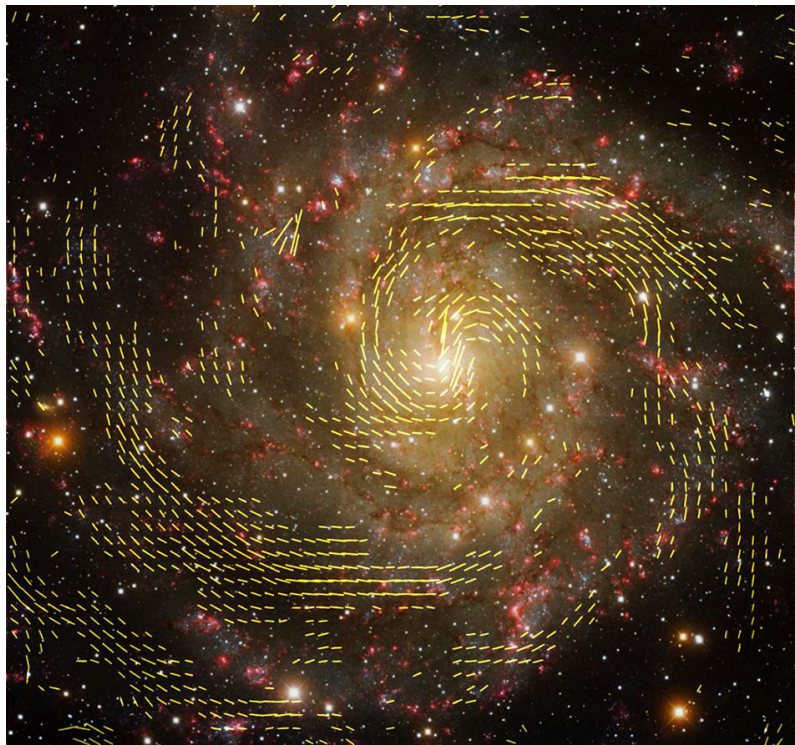

Fig. 6 Polarization B-vectors of IC 342, combined from observations at $6 \mathrm{~cm}$ wavelength with the VLA and Effelsberg telescopes and smoothed to $25^{\prime \prime}$ resolution (from Beck 2015), overlaid on a colour image from the Kitt Peak Observatory (credit: T.A. Rector, University of Alaska Anchorage, and H. Schweiker, WIYN and NOAO/AURA/NSF). A region of $16^{\prime} \times 16^{\prime}$ (about $16 \times 16 \mathrm{kpc}$ ) is shown. (Copyright: MPIfR Bonn)

Fig. 7 Polarized radio emission (contours) and B-vectors of M 83, combined from observations at $6 \mathrm{~cm}$ wavelength with the VLA and Effelsberg telescopes and smoothed to $15^{\prime \prime}$ resolution (from Frick et al. 2016), overlaid onto an optical image from Dave Malin, Anglo Australian Observatory. (Copyright: MPIfR Bonn and AAO)

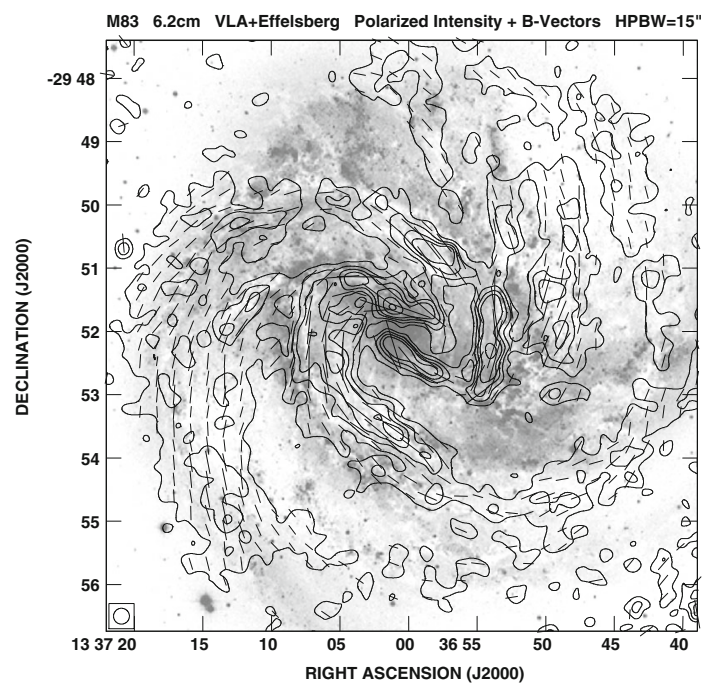

optical arm shows systematic variations in polarized emission, polarization angles and Faraday rotation measures on a scale of about $2 \mathrm{kpc}$, indicative of a helically twisted flux tube generated by the Parker instability (Sect. 4.10). Several broad polar- 
ization arms in the outer galaxy are coincident with spiral arms in the total neutral gas.

At wavelengths of around $20 \mathrm{~cm}$, most of the polarized emission from the far side of the disk and halo is Faraday-depolarized and the emission from the front side dominates. A striking asymmetry of the polarized emission occurs along the major axis of 12 spiral galaxies with inclinations of less than about $60^{\circ}$. The emission is always much weaker around the kinematically receding side (positive radial velocities) of the major axis (Urbanik et al. 1997; Braun et al. 2010; Vollmer et al. 2013). This asymmetry is still visible at $11 \mathrm{~cm}$ wavelength, but disappears at smaller wavelengths where the polarized emission from the far side becomes observable. In strongly inclined galaxies, both sides of the major axis become Faraday-depolarized at around $20 \mathrm{~cm}$. Modelling of a combination of disk and halo fields, as predicted by $\alpha-\Omega$ dynamo models (Sect. 2), can explain the asymmetry (Braun et al. 2010).

At even longer wavelengths, Faraday effects depolarize the synchrotron emission almost completely. With help of Faraday synthesis applied to $90 \mathrm{~cm}$ data from the Westerbork synthesis Radio Telescope, an extremely low average degree of polarization of $0.21 \pm 0.05 \%$ was measured in the star-forming "ring" of M 31 (Gießübel et al. 2013). No polarized emission could be detected from M 51 with the Low Frequency Array (LOFAR) at around $2 \mathrm{~m}$ wavelength-total Faraday depolarization occurs at such long wavelengths (Mulcahy et al. 2014).

\subsection{Spiral pitch angles}

In mean-field dynamo theory, the average pitch angle of the spiral magnetic field $p_{\mathrm{B}}$ and the average pitch angle of the gaseous spiral arms $p_{\mathrm{a}}$ are not related, while they are observed to be roughly similar in a limited sample of galaxies (Fig. 9). Within

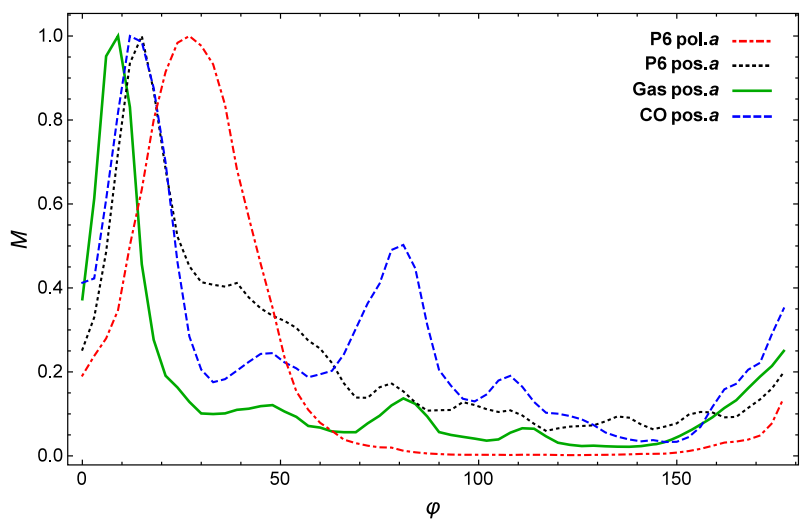

Fig. 8 The power spectra of pitch angles of structures at the wavelet-filtered scale $1^{\prime}$ (about $2.6 \mathrm{kpc}$ ) in M 83, normalized to unit maximum: the pitch angles of the structures of polarized intensity at $6 \mathrm{~cm}$ wavelength (black dotted), total neutral gas (green) and molecular gas $\mathrm{CO}$ (blue dashed). The spectrum of the magnetic pitch angles is shown with the red dot-dashed curve (from Frick et al. 2016) 
Fig. 9 Average magnetic pitch angles $\left|p_{\mathrm{B}}\right|$ and average pitch angles of gaseous spiral structures $\left|p_{\mathrm{a}}\right|$ in several radial regions of 12 galaxies. The dashed line corresponds to $\left|p_{\mathrm{B}}\right|=\left|p_{\mathrm{a}}\right|$ (from Van Eck et al. 2015)

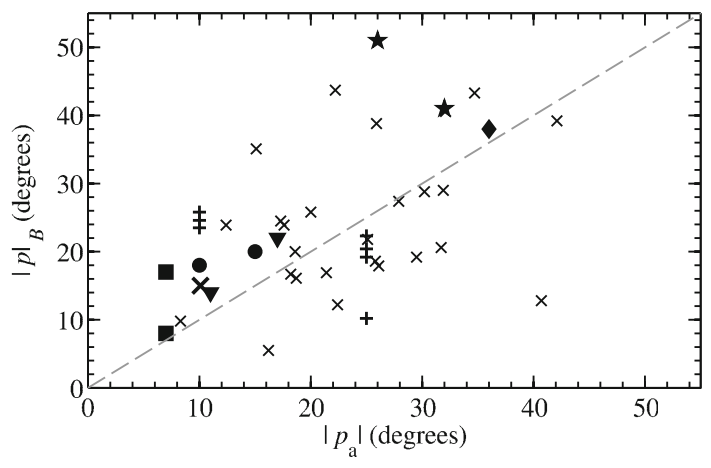

galaxies, too, the magnetic pitch angles are similar to the pitch angles of the gaseous spiral arm structures and of those of the polarized intensity, tracing the structures of the ordered field (e.g. Fig. 6). In IC 342 the magnetic pitch angle $\left|p_{\mathrm{B}}\right|$ decreases with radius (Fig. 10), following the pitch angle of the gaseous spiral structures (Beck 2015).

The formation of spiral arms and spiral magnetic patterns seems to be related, as expected, e.g. for MHD density waves (Lou et al. 1999). Almost identical pitch angles would indicate field alignment with the gas structures by shear or compression in density waves. However, based on anisotropic wavelet transformations, a systematic shift was detected in the barred galaxy M 83 (Fig. 7), in the sense that $\left|p_{\mathrm{B}}\right|$ is larger than $\left|p_{\mathrm{a}}\right|$ by about $20^{\circ}$ (Fig. 8). Similar results were found in other galaxies. Such differences can be regarded as a signature of $\alpha-\Omega$ dynamo action (Sect. 4.8).

In the thin-disk approximation of the $\alpha-\Omega$ dynamo, the magnetic pitch angle is given by $\left|p_{\mathrm{B}}\right|=\left(R_{\alpha} / R_{\Omega}\right)^{1 / 2}$, where $R_{\alpha}$ and $R_{\Omega}$ are the dynamo numbers (Shukurov 2005). Simplified estimates for the dynamo numbers and a flat rotation curve, which is valid beyond a radius of about a few kpc in most spiral galaxies, give $\left|p_{\mathrm{B}}\right|=d / H$, where $d$ is the turbulence scale (about $50 \mathrm{pc}$, see Fletcher et al. 2011), and $H$ is the scale height of the ionized gas. The scale heights of $\mathrm{H}$ I disks in spiral galaxies increase radially (Bagetakos et al. 2011). One may speculate that the disk of ionized gas also flares (e.g. assumed in the model by Gressel et al. 2013), which would explain the decrease of $\left|p_{\mathrm{B}}\right|$ observed in many galaxies. If so, the radial variation of $\left|p_{\mathrm{B}}\right|$ in IC 342 (Fig. 10) indicates that the scale height $H$ is approximately constant until $12^{\prime}$ (about $12 \mathrm{kpc}$ ) radius and then increases. Alternatively, the magnetic pitch angle may be affected by gas flows, for instance by outflows that become weaker towards the outer disk and can decrease the effective dynamo number (Shukurov et al. 2006).

\subsection{Regular fields}

Spiral fields can be generated by compression in spiral arms, by shear in interarm regions or by dynamo action (Sect. 2). Measuring Faraday rotation (Sect. 3.6) is crucial to distinguish between these mechanisms. Large-scale sinusoidal patterns of Faraday rotation measures (RM) along azimuthal angle in the galaxy plane (modes) are 
Fig. 10 Azimuthally averaged magnetic pitch angle $p_{\mathrm{B}}$ as a function of radius (in arcminutes) in the plane of IC 342. $p_{\mathrm{B}}$ is negative in this galaxy, because the spiral pattern (see Fig. 24) turns outward in the clockwise sense (from Beck 2015)

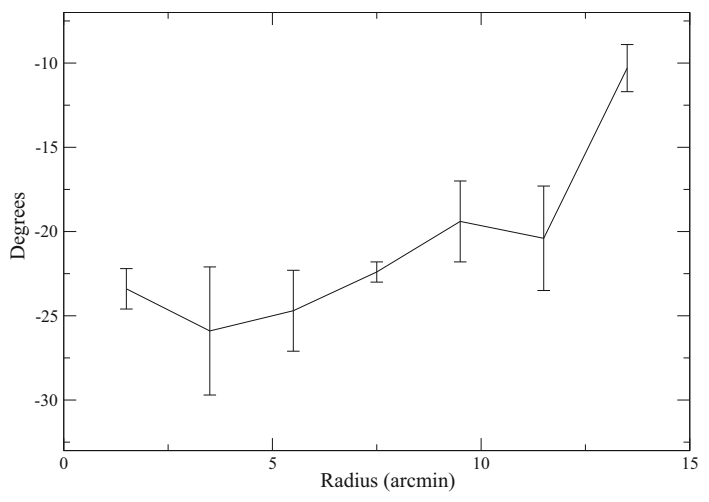

signatures of regular fields generated by the $\alpha-\Omega$ dynamo and can be identified in RM derived from polarized emission from the galaxy disks at several frequencies (Krause 1990) or in RM data of polarized background sources (Stepanov et al. 2008). If several modes are superimposed, a Fourier analysis of the RM variation with azimuthal angle is needed. The resolution and sensitivity of present-day radio observations is sufficient to identify 2-3 modes.

The disks of about a dozen nearby spiral galaxies reveal large-scale RM patterns. The Andromeda galaxy M 31 (Fig. 11) is the prototype of a dynamo-generated axisymmetric spiral disk field, with a striking sinusoidal RM variation along the star-forming ring (Fig. 12), which is a strong indication of an axisymmetric spiral field (mode $m=0$ ) (Fletcher et al. 2004). Other candidates for a dominating axisymmetric disk field are the nearby spirals IC 342 (Sokoloff et al. 1992; Beck 2015) and NGC 253 (Heesen et al. 2009b). The axisymmetric field in the irregular Large Magellanic Cloud (LMC) is almost azimuthal (i.e. small pitch angles) (Gaensler et al. 2005; Mao et al. 2012). Dominating bisymmetric spiral fields (mode $m=1$ ) are rare, as predicted by dynamo models, but possibly exist in M 81 (Krause et al. 1989; Sokoloff et al. 1992). Faraday rotation in NGC 6946 and in other similar galaxies with magnetic arms can be described by a superposition of two azimuthal modes ( $m=0$ and $m=2$ ) with about equal amplitudes, where the quadrisymmetric $(m=2)$ mode is phase shifted with respect to the density wave (Beck 2007). For several other galaxies, three modes ( $m=0,1$ and 2 ) are necessary to describe the data (Table 2).

The spiral pattern of magnetic fields cannot be solely the result of $\alpha-\Omega$ dynamo action. If the beautiful spiral pattern of M 51 seen in radio polarization (Fig. 5) were only due to a regular field in the disk, its line-of sight component should generate a conspicuous large-scale pattern in RM, but this is not observed (Fletcher et al. 2011). This means that a large amount of the ordered field is anisotropic turbulent and probably generated by compression and shear of the non-axisymmetric gas flows in the density-wave potential. The anisotropic turbulent field is strongest at the positions of the prominent dust lanes on the inner edge of the inner gas spiral arms, due to compression of turbulent fields in the density-wave shock. A regular field (composed of modes $m=0$ and $m=2$ ) also exists in the disk of M 51, but is much weaker than 


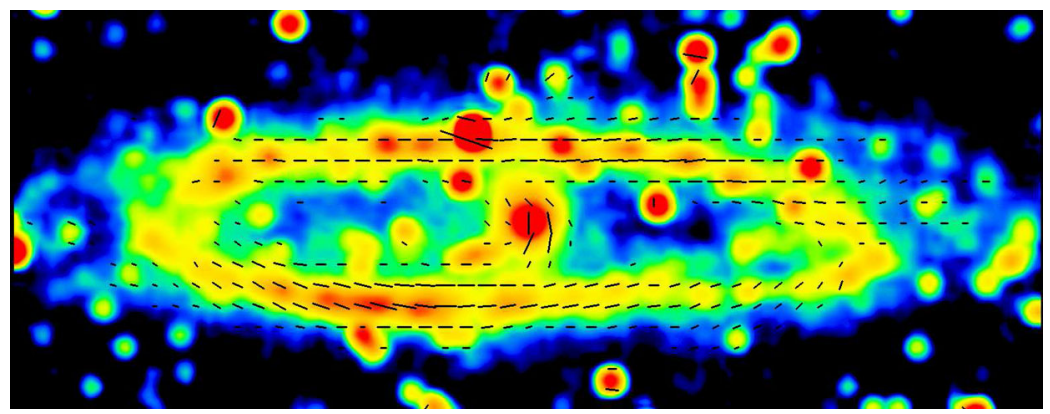

Fig. 11 Total radio emission (colour) and B-vectors (corrected for Faraday rotation in the foreground of the Milky Way) in the Andromeda galaxy (M 31), observed at $6 \mathrm{~cm}$ wavelength with the Effelsberg telescope at $3^{\prime}$ resolution (from Gießübel 2012). (Copyright: MPIfR Bonn)

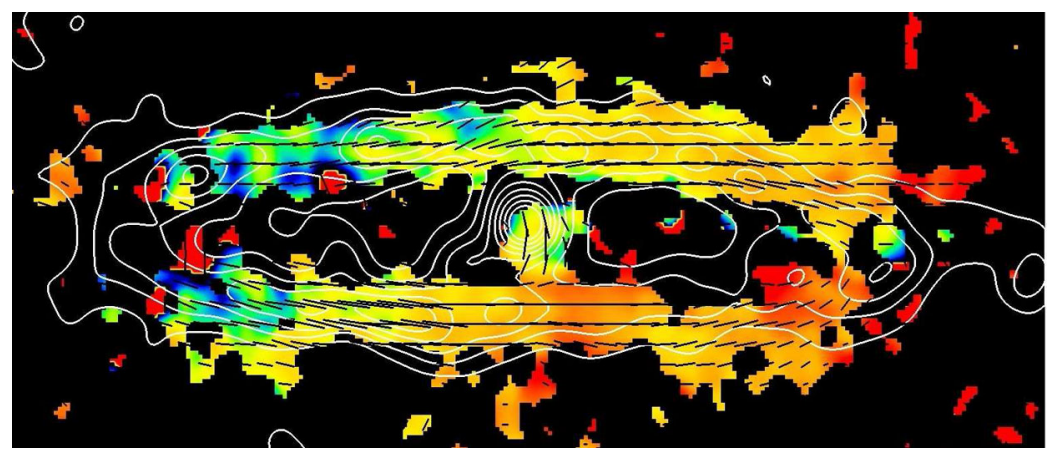

Fig. 12 Faraday rotation measures between 6 and $11 \mathrm{~cm}$ wavelengths (colour, from blue $=-175 \mathrm{rad} \mathrm{m}^{-2}$ to red $=+25 \mathrm{rad} \mathrm{m}^{-2}$ ), total radio emission at $6 \mathrm{~cm}$ (contours) and B-vectors (corrected for Faraday rotation) in the Andromeda galaxy (M 31), observed with the Effelsberg telescope at $5^{\prime}$ resolution (from Berkhuijsen et al. 2003). (Copyright: MPIfR Bonn)

the anisotropic turbulent field (Fletcher et al. 2011). Notably, RMs between 18 and $22 \mathrm{~cm}$ wavelengths, tracing only the polarized emission from regions nearest to the observer, show a large-scale pattern that indicates a regular field in the halo of M 51 (Heald et al. 2009; Fletcher et al. 2011; Mao et al. 2015).

The large-scale regular field in the nearby galaxy IC 342 is similarly weak as in M 51. It is only visible in the pattern of RMs obtained from the low-resolution Effelsberg images (Beck 2015), while RMs derived from the higher-resolution VLA images (Fig. 14) are about ten times larger. The field direction changes along the northern spiral arm (Sect. 4.10).

The central regions of M 31 (Fig. 11) and IC 342 (Fig. 17) host regular spiral fields that are disconnected from the disk fields (Gießübel and Beck 2014; Beck 2015). As the direction of the radial field component points outwards, opposite to that of the disk field, two separate dynamos seem to operate in these galaxies.

In the disks of many other galaxies no clear pattern of Faraday rotation was found. Either several high-order modes are superimposed and cannot be distinguished with 
Table 2 Decomposition of regular magnetic fields in galaxies into azimuthal modes of order $m$

\begin{tabular}{lllll}
\hline Galaxy & $m=0$ & $m=1$ & $m=2$ & References \\
\hline M 31 & 1 & 0 & 0 & Fletcher et al. (2004) \\
M 33 & 1 & 1 & 0.5 & Tabatabaei et al. (2008) \\
M 51 (disk) & 1 & 0 & 0.5 & Fletcher et al. (2011) \\
M 51 (halo) & 0 & 1 & 0 & Fletcher et al. (2011) \\
M 81 & 0.5 & 1 & - & Sokoloff et al. (1992) \\
NGC 253 & 1 & - & - & Heesen et al. (2009b) \\
NGC 1097 & 1 & 1 & 1 & Beck et al. (2005) \\
NGC 1365 & 1 & 1 & 1 & Beck et al. (2005) \\
NGC 4254 & 1 & 0.5 & - & Chyży (2008) \\
NGC 4414 & 1 & 0.5 & 0.5 & Soida et al. (2002) \\
NGC 6946 & $1 ?$ & - & $1 ?$ & Ehle and Beck (1993) and Rohde et al. (1999) \\
IC 342 & 1 & - & - & Sokoloff et al. (1992) and Beck (2015) \\
LMC & 1 & - & - & Gaensler et al. (2005) and Mao et al. (2012) \\
\hline
\end{tabular}

Columns 2-4 give the approximate amplitudes relative to the dominating mode. A dash indicates modes that were not sought, whereas a zero indicates that no signature of this mode was found. Modes of order higher than 2 cannot be detected with present-day telescopes. This is an updated version of a table in Fletcher (2010)

the limited sensitivity and resolution of present-day telescopes, or the timescale for the generation of large-scale modes is longer than the galaxy's lifetime (Arshakian et al. 2009). Also field injection by strong star-formation activity may perturb the generation of a large-scale regular field (Moss et al. 2012).

While the azimuthal symmetries of regular magnetic fields have been well observed in many galaxies (Table 2), the vertical symmetry (even or odd) is much harder to determine. The field of odd-symmetry modes reverses its sign above and below the galactic plane. The symmetry type is best visible in strongly inclined galaxies, via the RM signs above and below the galaxy plane. In mildly inclined galaxies the RMs of diffuse polarized emission from even and odd-symmetry fields differ by a factor of about two, which is hard to measure, while the RMs of background sources are close to zero for even symmetry (because the RM contributions from the two halves cancel) but large for odd symmetry. Background RMs in the area of the Large Magellanic Cloud (LMC) indicate an even-symmetry field (Mao et al. 2012). Indications for evensymmetry patterns were found in NGC 891 (Krause 2009) and NGC 5775 (Soida et al. 2011).

Spectro-polarimetric data of spiral galaxies are still rare and the application of Faraday synthesis (Sect. 3.6) has just started (e.g. Gießübel et al. 2013; Mao et al. 2015). The Faraday spectrum reflects the distribution of regular magnetic fields, thermal gas and CREs and in principle allows tomography of the ISM in the disk and the halo. However, Faraday spectra do not have a simple correspondence to physical properties and cannot provide a straight-forward interpretation, as demonstrated by the models of Ideguchi et al. (2014). 


\subsection{Testing dynamo models}

The following observations provide several qualitative evidences for the action of dynamos in spiral galaxies:

1. The tight radio-IR correlation (Sect. 4.4) and the similarity of kinetic and magnetic energy densities (Fig. 3) indicate field amplification by turbulent gas motions generated by star-forming processes (Tabatabaei et al. 2013b; Schleicher and Beck 2013).

2. The magnetic spiral patterns observed in all spiral galaxies so far indicate a general decoupling between magnetic fields and the (almost circular) gas flow due to magnetic diffusivity. The magnetic pitch angle deviates systematically from that of the spiral structures (e.g. in M 83, Fig. 8), as predicted by the $\alpha-\Omega$ dynamo. Magnetic spiral patterns also exist in flocculent galaxies (Sect. 4.14). At present, no other model can explain the magnetic spiral patterns in the various types of galaxies.

3. Large-scale regular fields are observed in all spiral galaxies so far (Table 2), an important result giving a strong hint to the action of $\alpha-\Omega$ dynamos. No alternative model exists so far.

4. By comparing the signs of the RM distribution and the velocity field on both sides of a galaxy's major axis, the inward and outward directions of the radial component of the axisymmetric spiral field can be distinguished (Krause and Beck 1998). Dynamo models predict that both signs should have the same probability, which is confirmed by observations. The axisymmetric fields of M 31, IC 342, NGC 253 and the axisymmetric field component in NGC 6946 point inwards, while those of NGC 891, NGC 4254, NGC 5775 (Krause 2009) and the axisymmetric component of the disk field in M 51 (Fletcher et al. 2011) point outwards.

A major effort of quantitative comparisons between observable quantities and predictions was performed by Van Eck et al. (2015). Identifying the precise form of the connection between magnetic and other galactic properties is not straightforward because the parameters can combine to produce non-trivial scalings. For example, different physical mechanisms have been proposed to provide saturation of the field growth in dynamo models, like the balance between magnetic and kinetic (turbulent) forces, the balance between Coriolis and Lorentz forces, or outflows of hot gas carrying small-scale magnetic fields out of the disk to preserve the balance of magnetic helicity. These non-linear saturation mechanisms depend differently on galactic parameters, such as star-formation rate, gas density, rotational velocity and rotational shear.

From their sample of 20 well-observed galaxies, Van Eck et al. (2015) found a statistically significant relation of the total magnetic field strength with the surface density of molecular gas and surface density of the star-formation rate, confirming earlier results (Sect. 4.4). The only other significant relation was found between the magnetic pitch angle and the strength of the axisymmetric spiral $(m=0)$ component of the regular field: a more tightly wound field has a stronger ASS component. However, IC 342 does not fit into this relation; has a tightly wound spiral field (Fig. 10), but only a weak axisymmetric component (Beck 2015). 
In conclusion, dynamos certainly operate in all spiral galaxies, but other processes are amplifying and shaping the field as well. MHD density waves are compressing and aligning the field along the gaseous spiral arms. Non-axisymmetric gas flows around spiral arms and bars are shearing field lines. Parker instabilities form helically twisted field loops that are winding around spiral arms. To measure the importance of these competing or cooperating effects, the fundamental scaling relations between the properties of magnetic fields and the other galactic parameters need to be determined, based on radio observations of a large number of galaxies with high angular resolution.

\subsection{Magnetic arms}

Surprisingly, in most spiral galaxies observed so far the highest polarized intensities (i.e. the strongest ordered fields) are detected between the optical arms, filling a large fraction of the interarm space, sometimes concentrated in magnetic arms, as in NGC 6946 (Fig. 13). The southern-sky spiral galaxy NGC 2997 hosts compressed magnetic fields at the inner edges of material arms, as well as one well-developed magnetic arm (Han et al. 1999). The strong density waves in NGC 2997 lead to high degrees of polarization at $6 \mathrm{~cm}$ wavelength of typically $25 \%$ at the inner edge of the northern arm, similar to those in M 51 (Fletcher et al. 2011). The degree of polarization of the magnetic arm of $40 \%$ and its length of at least $10 \mathrm{kpc}$ are similar to the values in NGC 6946 and are much higher than in IC 342 (Beck 2015). Like IC 342, M 51 has a short, rudimentary magnetic arm with a low degree of polarization (Fletcher et al. 2011). NGC 1566 shows signatures of a magnetic arm in the south-east (Ehle et al. 1996), but the angular resolution of these radio observations was too coarse to clearly detect magnetic arms. In large barred galaxies, ordered magnetic fields also fill most of the interarm space, e.g. in NGC 2442 (Harnett et al. 2004), NGC 1097 and NGC 1365 (Beck et al. 2005), but do not form well-defined magnetic arms.

Several mechanisms have been proposed to explain the high degree of field order in interarm regions:

1. Magnetic field ropes as a result of a magnetic buoyancy instability in a turbulent high- $\beta$ plasma (Kleeorin and Rogachevskii 1990); however, the ISM of galaxies is a low- $\beta$ plasma, meaning that the thermal pressure is lower than the magnetic pressure (Sect. 4.2);

2. slow MHD density waves in the rigidly rotating inner region of a galaxy (Lou et al. 1999; Poedts and Rogava 2002); however, slow MHD density waves in 3D can be subject to the Parker and shearing instabilities (Foglizzo and Tagger 1995);

3. more efficient action of the mean-field dynamo between the optical arms due to lower turbulent velocity in interarm regions (Moss 1998; Shukurov 1998); however, the observed turbulent velocity is not lower in the interarm regions (e.g. in IC 342, Crosthwaite et al. 2001);

4. introduction of a relaxation time of the magnetic response in the dynamo equation, leading to a phase shift between the material and magnetic spiral arms (Chamandy et al. 2013a, b); however, the resulting magnetic arms are restricted to a relatively small region around the corotation radius and have a much smaller pitch angle than the gaseous arms; 


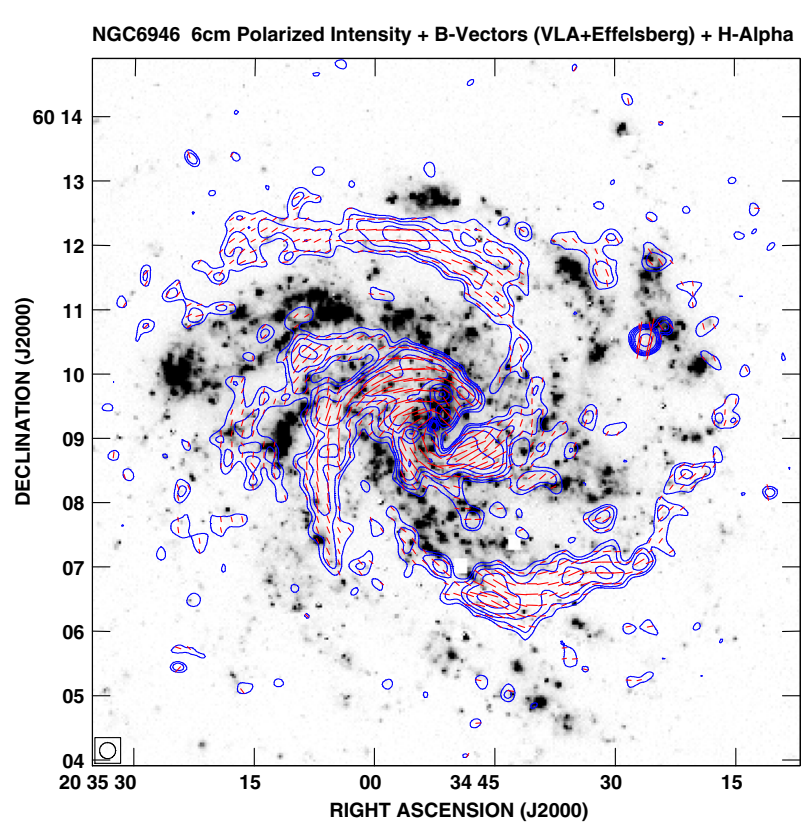

Fig. 13 Polarized radio emission (contours) and B-vectors of NCC 6946, combined from observations at $6 \mathrm{~cm}$ wavelength with the VLA and Effelsberg telescopes and smoothed to $15^{\prime \prime}$ resolution (from Beck 2007), overlaid onto an $H \alpha$ image from Anne Ferguson. (Copyright: MPIfR Bonn)

5. drift of magnetic fields with respect to the gaseous arms in a non-axisymmetric gas flow caused by a spiral perturbation (Otmianowska-Mazur et al. 2002) or by a bar (Kulpa-Dybeł et al. 2011);

6. weakening of the mean-field dynamo in the material arms by continuous injection and amplification of turbulent fields by supernova shock fronts (Moss et al. 2013, 2015);

7. weakening of the mean-field dynamo in the material arms by star-formation-driven outflows (Chamandy et al. 2015);

8. magnetic arms as a transient phenomenon during the evolution of galactic magnetic fields, possibly related to the short lifetimes of spiral patterns seen in numerical simulations (e.g. Dobbs and Baba 2014).

Models (5)-(8) are promising. Still, these models are simplified and consider either gravitational perturbations or dynamo action. Self-consistent MHD models of galaxies including the gravitational potential with spiral perturbations and mean-field dynamo action are still missing.

The origin of the rudimentary magnetic arm in IC 342 (Fig. 6) deserves a detailed discussion. The absence of long magnetic arms in IC 342 is surprising in view of the apparent similarities between IC 342 and NGC 6946, like the rotation curve and the star-formation surface density $\Sigma_{\mathrm{SFR}}$ (Calzetti et al. 2010). The evolution of a spiral magnetic pattern in IC 342 may be hampered by the possible tidal interaction with the Local Group (Buta and McCall 1999) and/or by the complex spiral pattern in the gas of IC 342, described by a superposition of a two- and four-armed spiral pattern 
with different pattern speeds (Meidt et al. 2009), which may lead to an unstable spiral pattern. The mean-field dynamo needs a least a few rotation periods to build up a regular field (e.g. Moss et al. 2012). A short lifetime of a stable pattern may not allow the formation of magnetic arms.

The spiral pattern in the gas of M 51 is also distorted and short-lived so that only a weak large-scale regular field and a rudimentary magnetic arm could develop. On the other hand, NGC 6946 has a two-armed spiral pattern with a well-defined pattern speed (Fathi et al. 2007), the spiral field extends smoothly into the central region (Beck 2007) and the large-scale regular field is strong (Ehle and Beck 1993). In summary, the existence of magnetic arms may indicate a stable spiral arm pattern over several galactic rotation periods, which may not be given in IC 342.

Most models of spiral gravitational perturbations in stars and gas have neglected the effect of magnetic fields so far. On the other hand, the only dynamo model for the amplification and ordering of magnetic fields including the spiral perturbations of gas density and gas velocity is the kinematical model by Otmianowska-Mazur et al. (2002), in which the back reaction of the field onto the gas flow was not included. The MHD model of Pakmor and Springel (2013) includes self-gravity and spiral perturbations, but no mean-field dynamo action. There is urgent need for a synergy between these approaches to achieve a comprehensive description of the evolving magnetized ISM in galaxies.

\subsection{Helically twisted Parker loops}

IC 342 is one of the best studied nearby spiral galaxies. Its small distance and bright radio disk allow detailed studies of the magnetic field that are still impossible in most other galaxies.

Faraday rotation measures (Fig. 14) are found to vary periodically along the most prominent northern radio spiral arm. The average distance between the extrema corresponds to about $2.1 \mathrm{kpc}$. This feature can be interpreted as a magnetic loop (Parker instability) extending out of the galaxy's disk and out of the sky plane, giving rise to a periodic pattern in RM. A regular field bending out of the plane should lead to minima in polarized intensity (tracing the field component in the sky plane) at locations where $|R M|$ (tracing the field component along the line of sight) is at its maximum. Minima of polarized intensity occur roughly at the locations of the extrema in RM, with a similar distance between the maxima or minima of about $2.2 \mathrm{kpc}$.

This is the second indication of a Parker loop in the magnetic field of a nearby galaxy, after M 31 (Beck et al. 1989). The numerical models by Kim et al. (2002) (who assume $\beta=1$ ) predict a wavelength of the most unstable symmetric mode between $4 \pi H$ and $17 H$, where $H$ is the scale height of the gas. The peak-to-peak wavelength of about $4 \mathrm{kpc}$ measured in IC 342 corresponds to $H \approx 230-320 \mathrm{pc}$, which is larger than typical scale heights of $\mathrm{H}$ I gas disks of spiral galaxies (Bagetakos et al. 2011). The reason for this discrepancy may be that the assumption of $\beta=1$ is incorrect, because observations indicate $\beta<1$ (Sect. 4.2).

The magnetic field in the northern arm of IC 342 diverts not only in the vertical direction, but also in the disk plane. The ridge line of the polarization spiral arm 
Fig. 14 Faraday rotation measures (RM) between 3.5 and $6 \mathrm{~cm}$ wavelengths (colour) and total emission at $6 \mathrm{~cm}$ (contours) in the central and northern regions of IC 342, observed with the VLA at $25^{\prime \prime}$ resolution. RM is shown only at points where the polarized intensities at both wavelengths exceeds three times the rms noise (from Beck 2015)

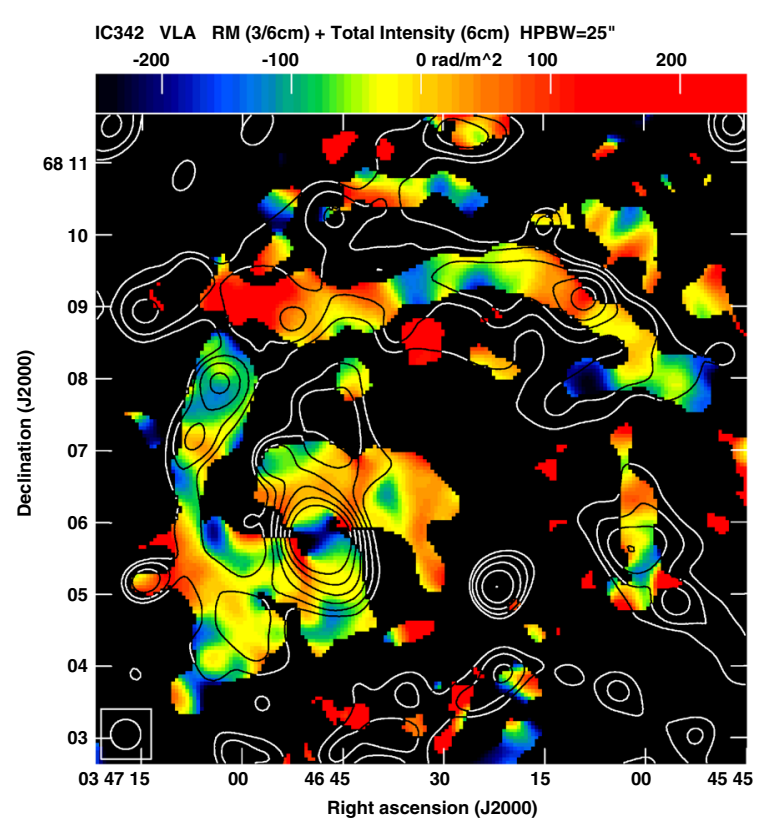

as well as the magnetic pitch angle (Fig. 6) oscillate around the northern arm with similar periodicity. This gives indication for a large-scale, helically twisted flux tube, as predicted by models of the Parker loops under the Coriolis force (Shibata and Matsumoto 1991; Hanasz et al. 2002).

In the galaxy NGC 7479 (Fig. 24), two jets serve as bright polarized background sources. High-resolution observations revealed several reversals in RM on 1-2 kpc scale, originating in the foreground disk of the galaxy (Laine and Beck 2008), which may represent another case of a helically twisted field loop.

\subsection{Large-scale field reversals}

A large-scale field reversal exists between the central region and the disk in M 31 (Gießübel and Beck 2014) and in IC 342 (Beck 2015). A large-scale field reversal at about constant azimuthal angle across the whole galaxy disk was found in NGC 4414 (Soida et al. 2002). To fulfil the divergence-free condition, this reversal cannot occur in the same volume, but, e.g. between disk and halo. Indication for a large-scale field reversal between disk and halo was also found in M 51 (Berkhuijsen et al. 1997; Fletcher et al. 2011; Mao et al. 2015). New observations and applying Faraday synthesis (Sect. 3.6) are needed.

Large-scale field reversals between spiral arms, like that observed in the Milky Way (Fig. 26), have not been detected in external galaxies, although high-resolution images of Faraday rotation are available for many spiral galaxies. Possible reasons are discussed in Sect. 5. 


\subsection{Barred galaxies}

Galaxies with massive bars have non-axisymmetic gas flows that interact with magnetic fields. The magnetic field lines in NGC 1097, one of the closest and brightest barred galaxies (Fig. 15), follow a pattern that resembles the flow of cold gas in a bar potential. As the gas rotates faster than the bar pattern of a galaxy, a shock occurs in the cold gas, which has a small sound speed, while the flow of warm, diffuse gas is only slightly compressed but sheared. The ordered field is also hardly compressed, probably coupled to the diffuse gas and strong enough to affect its flow (Beck et al. 2005). The ordered field is also strong in the upstream region (south of the centre in Fig. 15), oriented almost perpendicularly to the bar and aligned with the dust filaments seen on the optical image in the background. The polarization pattern in barred galaxies can be used as a tracer of shearing gas flows in the sky plane and complements spectroscopic measurements of radial velocities.

The central regions of barred galaxies are often sites of ongoing intense star formation and strong magnetic fields that can affect gas flows. NGC 1097 hosts a bright ring with about $1.5 \mathrm{kpc}$ diameter and an active nucleus in its centre (Fig. 16). The ordered field in the ring has a spiral pattern and extends to the nucleus. The orientation of the innermost spiral field agrees with that of the spiral dust filaments visible on optical

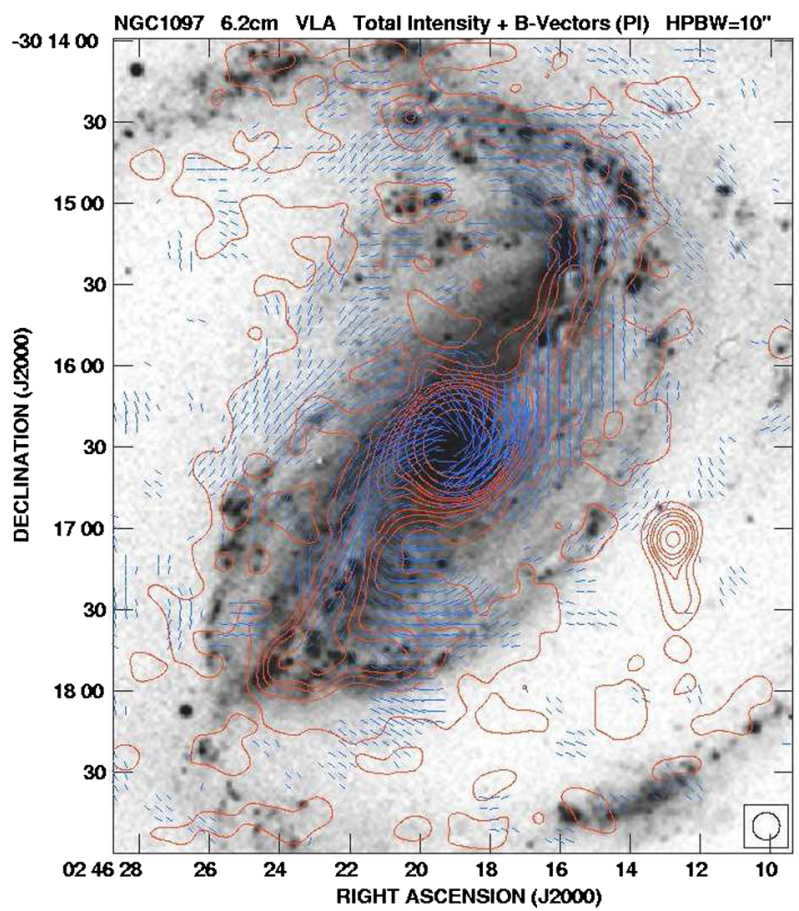

Fig. 15 Total radio emission (contours) and B-vectors of the barred galaxy NGC 1097, observed at $6 \mathrm{~cm}$ wavelength with the VLA and smoothed to $10^{\prime \prime}$ resolution (from Beck et al. 2005). The background optical image is from Halton Arp 
Fig. 16 Total radio emission (contours) and B-vectors in the circumnuclear ring of the barred galaxy NGC 1097, observed at $3.5 \mathrm{~cm}$ wavelength with the VLA at $3^{\prime \prime}$ resolution. The background optical image is from the HST (from Beck et al. 2005)

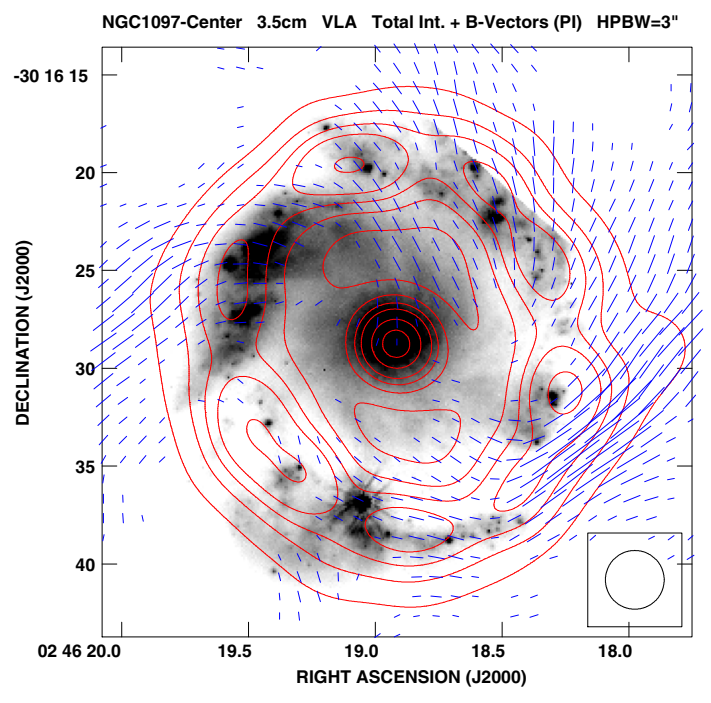

images. Magnetic stress in the circumnuclear ring due to the strong total magnetic field (about $50 \mu \mathrm{G}$ ) can drive gas inflow (Balbus and Hawley 1998) at a rate of several solar masses per year, which is sufficient to fuel the activity of the nucleus (Beck et al. 2005). MHD modelling confirmed that magnetic fields can strongly enhance the mass gas inflow rate (Kim and Stone 2012).

The central region of IC 342 hosts a bar of dust and cold gas with a wealth of molecular lines. In terms of size, dynamical mass, molecular mass and star-formation rate, the nucleus of IC 342 is a potential twin of the Galactic centre (Meier 2014). While the central bar in total radio intensity coincides with the central bar in the CO line emission from molecular gas, the polarized emission reveals a double-lobe structure that is displaced from the $\mathrm{CO}$ bar (Fig. 17).

\subsection{Magnetic halos}

Hot gas, magnetic fields and cosmic rays in galaxy disks drive outflows that extend far beyond the galaxy disks seen at optical wavelengths and form thick disks or halos at radio wavelengths. ${ }^{5}$ Radio polarization observations of several edge-on galaxies reveal vertical field components in the halo forming an X-shaped pattern, like in NGC 253 (Heesen et al. 2009b), NGC 891 (Fig. 18), NGC 4631 (Fig. 20) and NGC 5775 (Fig. 19), which may be related to outflows or to dynamo action (Moss et al. 2010) driven by such outflows.

The vertical profile of the total radio continuum emission gives information about the outflow speed. The profiles of five edge-on spiral galaxies observed with high resolution can be described by two exponential scale heights $H_{\text {syn }}, 300 \pm 50 \mathrm{pc}$ for the thin disk and $1.8 \pm 0.2 \mathrm{kpc}$ for the halo (Krause 2014). Neglecting the thermal

\footnotetext{
5 As a definition of how to distinguish "thick disk" from "halo" is missing, "halo" is used in the following.
} 
Fig. 17 Polarized intensity and B-vectors in the central region of IC 342 , observed at $3.5 \mathrm{~cm}$ wavelength with the VLA at $12^{\prime \prime}$ resolution, overlaid on a greyscale presentation of the $\mathrm{CO}(1-0)$ emission at $2.6 \mathrm{~mm}$ from the BIMA survey (Helfer et al. 2003)

Fig. 18 Total radio emission and B-vectors of the edge-on galaxy NGC 891, a galaxy similar to the Milky Way, observed at $3.6 \mathrm{~cm}$ wavelength with the Effelsberg telescope at $84^{\prime \prime}$ resolution (from Krause 2009). The background optical image is from the CFHT.

(Copyright: MPIfR Bonn)

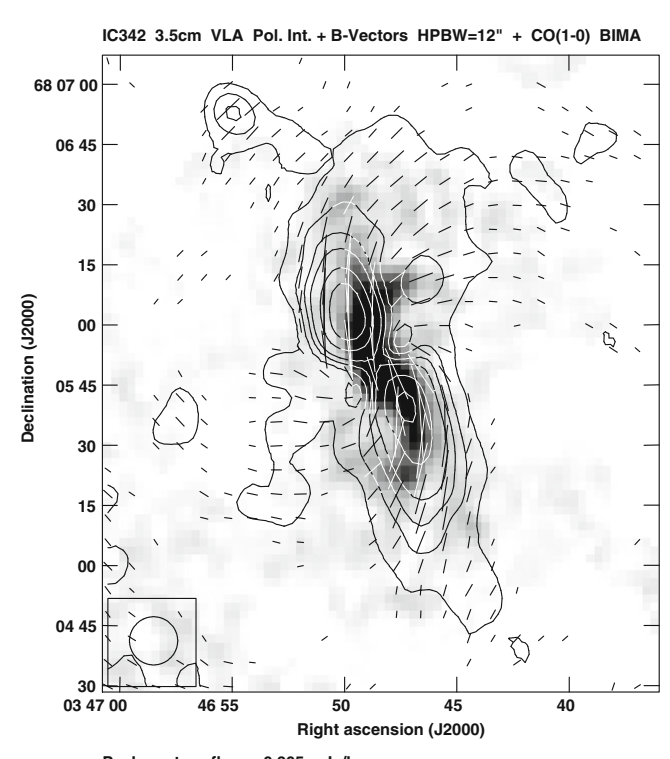

Peak contour flux $=0.305 \mathrm{mJy} / \mathrm{beam}$ Levs $=0.03 \mathrm{mJy} / \mathrm{beam} *(1,2,3,4,6,8)$
Pol line 1 arcsec $=12.5$ MicroJy/beam

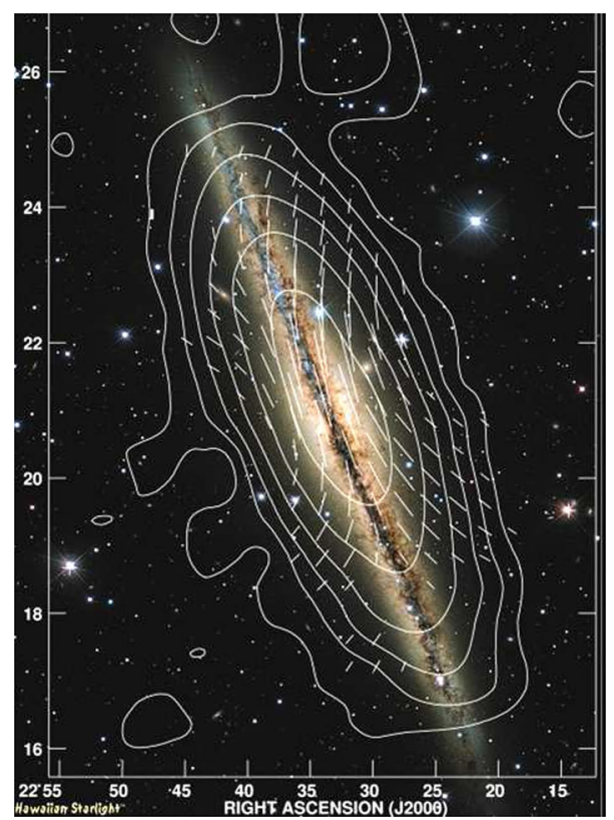

contributions and assuming energy equipartition between total magnetic fields and total cosmic rays (Sect. 3.4), the scale heights of the total magnetic field are typically $H_{\mathrm{B}} \geq(3+\alpha) H_{\text {syn }} \simeq 1.2 \mathrm{kpc}$ for the thin disk and about $7 \mathrm{kpc}$ for the halo, but probably larger due to energy losses of CREs. Because the average field strengths and 


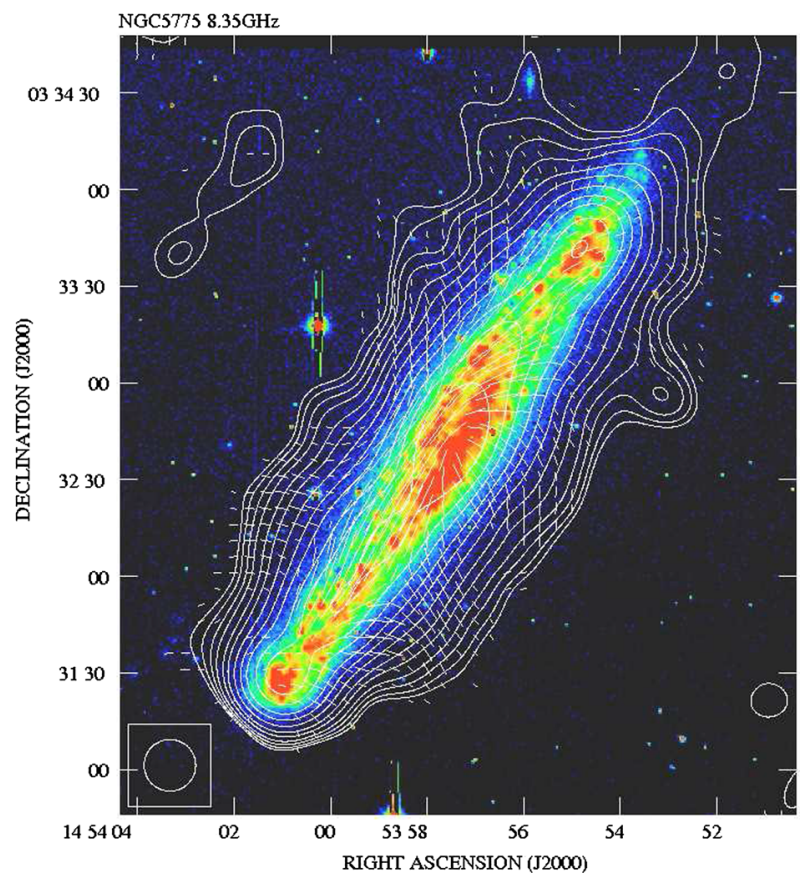

Fig. 19 Total radio intensity and B-vectors of the edge-on galaxy NGC 5775, combined from observations at $3.6 \mathrm{~cm}$ wavelength with the VLA and Effelsberg telescopes at 16" resolution (from Soida et al. 2011). The background $H \alpha$ image is from Tüllmann et al. (2000). (Copyright: MPIfR Bonn)

hence the synchrotron lifetimes of CREs are different in different galaxies, the roughly constant halo scale heights indicate that outflow speeds increasing with the average field strength (and hence with the star-formation rate) may balance the smaller CRE lifetimes (Krause 2009).

Stronger magnetic fields in the central regions cause higher synchrotron loss of CREs, leading to the "dumbbell" shape of many radio halos, e.g. in NGC 253 (Heesen et al. 2009a) and NGC 4565 (Krause 2009). From the radio scale heights at several frequencies and the corresponding lifetimes of CREs (depending on synchrotron, inverse Compton and adiabatic losses) a wind speed of about $300 \mathrm{~km} / \mathrm{s}$ was measured for the electrons in the halo of NGC 253 (Heesen et al. 2009a).

Galaxies without a detectable radio halo are rare. UGC 10288 shows discrete highlatitude radio features, but it does not have a global radio continuum halo (Irwin et al. 2013). No radio halo was found in M 31 (Berkhuijsen et al. 2013) and in NGC 7462 (Heesen et al., MNRAS, in press). These three galaxies have a star-formation rate that is probably too low to drive outflows.

On the other hand, exceptionally large and almost spherical radio halos are observed around interacting galaxies, namely NGC 4631 (Fig. 20) and the starburst galaxy M 82 (Adebahr et al. 2013; Reuter et al. 1994). A few magnetic spurs could be resolved in NGC 4631, connected to star-forming regions (Golla and Hummel 1994). These observations support the ideas of a strong galactic outflow (galactic wind), driven 


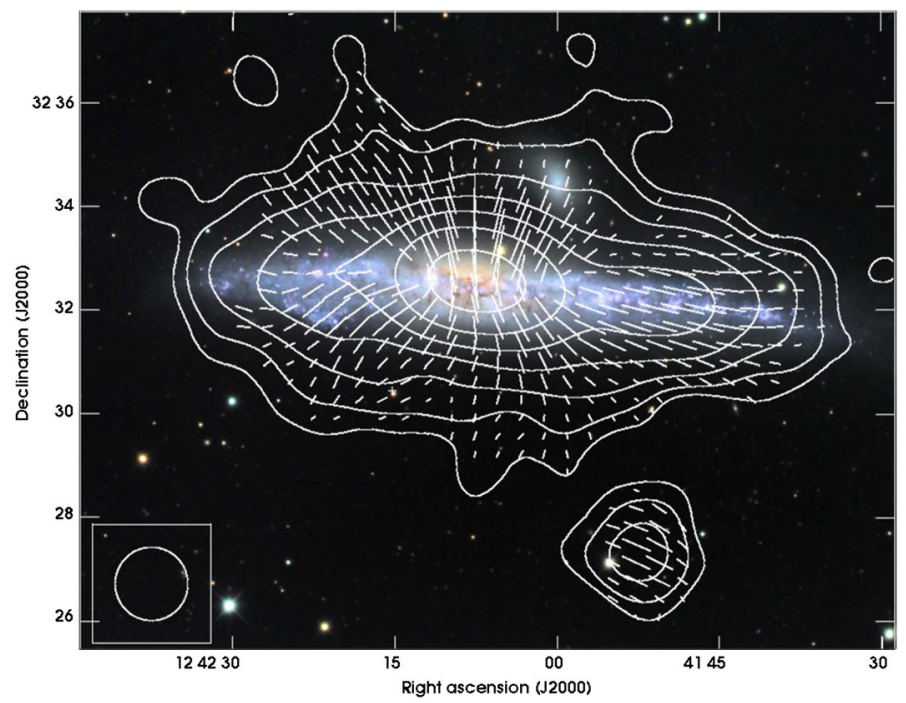

Fig. 20 Total radio emission (contours) and B-vectors of the edge-on galaxy NGC 4631, observed at $3.6 \mathrm{~cm}$ wavelength with the Effelsberg telescope at $85^{\prime \prime}$ resolution (from Mora and Krause 2013). The background optical image is from the Digital Sky Survey. (Copyright: MPIfR Bonn)

by regions of star formation in the disk, possibly supported by the interaction with neighbouring galaxies.

Above the central starburst region of NGC 253, an outwards-directed helical field of about $20 \mu \mathrm{G}$ strength, extending to at least $1 \mathrm{kpc}$ height, could be identified in the gas outflow cone with help of high-resolution RM mapping (Heesen et al. 2011a). This field may help to confine the outflow.

In summary, the occurrence of radio halos is governed by the competition between vertical transport of CREs by diffusion and/or convection (galactic wind) and energy losses of CREs, dominated by synchrotron loss. CRE transport can be characterized by the escape time $t_{\text {esc }}$ needed to reach the scale height $H$ of the disk, beyond which the synchrotron emission significantly weakens. In case of diffusive transport, $t_{\text {esc }} \propto$ $\left(H^{2} / D\right)$, where $D$ is the diffusion coefficient, while in case of convective transport $t_{\text {esc }} \propto H / v_{\text {wind }}$, where $v_{\text {wind }}$ is the average wind speed.

For strong magnetic fields or slow winds or slow diffusion $\left(t_{\text {syn }} \ll t_{\text {esc }}\right)$, the CREs cannot leave the disk and no radio halo develops, as in the case of M 31 (Berkhuijsen et al. 2013). For $t_{\text {syn }} \lesssim t_{\text {esc }}$, the scale height $H$ of the synchrotron halo increases with distance from the centre according to the increase in synchrotron lifetime ("dumbbell" halo) and with radio wavelength ( $H \propto \lambda^{1 / 4}$ for diffusive or $H \propto \lambda^{1 / 2}$ for convective propagation). For weak fields or fast winds $\left(t_{\mathrm{syn}}>t_{\mathrm{esc}}\right)$, a large radio halo of elliptical or spherical shape is formed with a wavelength-independent scale height that is determined by the scale height of the total magnetic field and adiabatic losses of the expanding CRE flow. ${ }^{6}$

\footnotetext{
6 Detailed models of radio halos are presented in Lerche and Schlickeiser (1982a, b).
} 


\subsection{Rudimentary spirals: flocculent and irregular galaxies}

Flocculent galaxies have disks but no gaseous spiral arms. Nevertheless, magnetic spiral patterns have been observed in all flocculent galaxies so far, indicating that the $\alpha-\Omega$ dynamo works independently of spiral density waves. Ordered magnetic fields with strengths similar to those in grand-design spiral galaxies have been detected in the flocculent galaxies M 33 (Tabatabaei et al. 2008), NGC 3521 and NGC 5055 (Knapik et al. 2000), and in NGC 4414 (Soida et al. 2002). The mean degree of polarization (corrected for the differences in spatial resolution) is also similar in grand-design and flocculent galaxies.

Radio continuum images of irregular, slowly rotating galaxies may reveal strong total magnetic fields, e.g. in the Magellanic-type galaxy NGC 4449 (Fig. 21), with a partly ordered field of about $7 \mu \mathrm{G}$ strength, a spiral pattern in the northeast and a radial pattern in the central region (Chyży et al. 2000). Faraday rotation data shows that this ordered field is partly regular and the $\alpha-\Omega$ dynamo is operating (Siejkowski et al. 2014).

The Large and the Small Magellanic Clouds are the closest irregular galaxies at distances of 50 and $60 \mathrm{kpc}$, respectively, interacting with the Milky Way and each other. Radio observations found the two galaxies to be weakly polarized (Haynes et al. 1991; Mao et al. 2012), with the exception of two highly polarized filaments in the southeast of the LMC. A study based on RM grids suggests that both galaxies host large-scale coherent fields, indicating that a large-scale dynamo also works under the less favourable conditions of slow ordered rotation (Mao et al. 2008, 2012). Due to

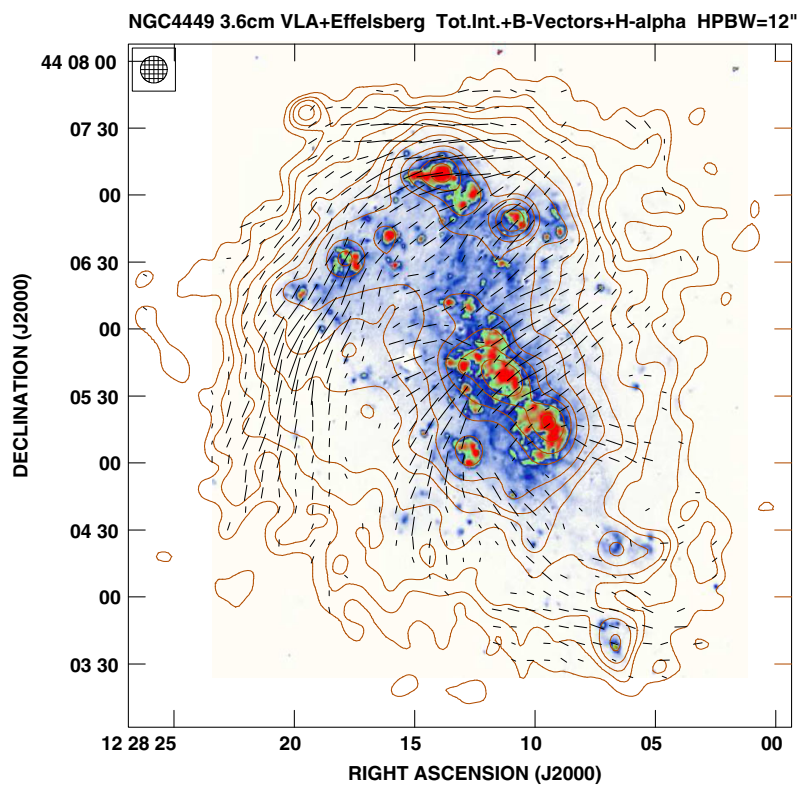

Fig. 21 Total radio emission (contours) and B-vectors of the dwarf irregular galaxy NGC 4449, observed at $3.6 \mathrm{~cm}$ wavelength with the VLA at $12^{\prime \prime}$ resolution (from Chyży et al. 2000). The background $H \alpha$ image is from Dominik Bomans (Bochum University) 
their low potential well, low-mass galaxies are prone to outflows and galactic winds driven by star formation.

In dwarf irregular galaxies, the strengths of the total (turbulent) field are generally smaller than in spiral galaxies (Chyży et al. 2011), except for starburst dwarfs, e.g. NGC 1569 with 10-15 $\mu \mathrm{G}$ total field strength (Kepley et al. 2010), where star formation activity is sufficiently high for the operation of the small-scale dynamo. Consequently, dwarf galaxies form the low-luminosity tail of the radio-IR correlation for large galaxies (Chyży et al. 2011).

Spots of faint polarized emission were detected in dwarf irregular galaxies, but no large-scale regular fields so far (Heesen et al. 2011b), either due to a lack of telescope sensitivity or a lack of dynamo action. In classical $\alpha-\Omega$ dynamo models, the dynamo number is too low for dynamo action because rotation is almost chaotic, while models including support of dynamo action by outflows predict $\alpha-\Omega$ dynamos even in dwarf galaxies (Rodrigues et al. 2015).

\subsection{Beyond spirals: interacting galaxies}

Gravitational interaction between galaxies leads to asymmetric gas flows, compression, shear, enhanced turbulence and outflows that can be traced by observing magnetic fields in radio polarization. Magnetic fields can become aligned along the compression front or perpendicular to the velocity gradients. Such gas flows make turbulent fields highly anisotropic. Large-scale dynamos can be enhanced by ram pressure (Moss et al. 2014).

The classical interacting galaxy pair is NGC 4038/39, the "Antennae" (Chyży and Beck 2004). It shows bright, extended radio emission filling the whole system. In the interaction region between the galaxies, where star formation did not yet start, and at the northeastern edge of the system, the magnetic field is partly ordered, probably the result of compression and shearing motions along the tidal tail. Particularly strong, almost unpolarized emission comes from a region of violent star formation, hidden in dust. The average total magnetic field is stronger than that in normal spirals, but the mean degree of polarization is unusually low, implying that the fields are tangled. The Antennae galaxies have been modelled by Kotarba et al. (2010), who could reproduce the main features observed in the radio image.

The total magnetic field in a sample of 16 systems in different interaction stages increases with advancing interaction, which indicates enhanced production of random magnetic fields (Drzazga et al. 2011). The strength of the ordered magnetic field are sensitive tools for revealing global galactic distortions. The pattern of the ordered field traces the orientation of gas flow in tidally stretched spiral arms and in tidal tails. Such outflows may have contributed to the magnetization of the intergalactic medium in the early cosmological epoch.

Interaction with a dense intergalactic medium also imprints unique signatures onto magnetic fields and thus onto the radio emission. The Virgo cluster is a location of especially strong interaction effects (Fig. 22), and almost all cluster galaxies observed so far show asymmetries of their polarized emission because the outer magnetic fields were compressed (Vollmer et al. 2007, 2013; Weżgowiec et al. 2007, 2012). Ordered 
fields are an excellent tracer of past interactions between galaxies or with the intergalactic medium.

\subsection{Spiral galaxies with jets}

Nuclear jets are observed in only a few spiral galaxies. These jets are weak and small compared to those of radio galaxies and quasars. Detection is further hampered by the fact that they emerge at some angle with respect to the disk so that little interaction with the ISM occurs. If the nuclear disk is oriented at a large angle to the disk, the jet hits a large amount of ISM matter, CREs are accelerated in shocks and the jet becomes radio-bright. However, not all jets are consistent with this morphology.

NGC 4258 is one of the rare cases where large radio jets of at least $15 \mathrm{kpc}$ length are observed (Fig. 23). A nuclear disk is observed in water maser emission, has an inner radius of $0.13 \mathrm{pc}$ and is seen almost edge-on (Greenhill et al. 1995). As the jets emerge perpendicular to the nuclear disk, they have to pass the galactic disk at a rather small angle. The magnetic field orientation is mainly along the jet direction. The equipartition field strength is about $300 \mu \mathrm{G}$ at the resolution of about $100 \mathrm{pc}$ (Krause and Löhr 2004), which is a lower limit due to energy losses of the CREs and the limited resolution.

The barred galaxy NGC 7479 also shows remarkable jet-like radio continuum features: bright, narrow, $12 \mathrm{kpc}$ long in projection and containing an aligned magnetic field (Fig. 24). The lack of any optical or near-infrared emission associated with the jets suggests that at least the outer parts of the jets are extraplanar features, although close to the disk plane. The equipartition strength is $35-40 \mu \mathrm{G}$ for the total magnetic field and about $10 \mu \mathrm{G}$ for the ordered magnetic field in the jets. According to Faraday rotation

Fig. 22 Polarized radio emission (contours) and B-vectors of the Virgo galaxy NGC 4535 , observed at $3.6 \mathrm{~cm}$ wavelength with the Effelsberg telescope at $90^{\prime \prime}$ resolution (from Weżgowiec et al. 2012 and priv. comm.). The background optical image is from the Digital Sky Survey

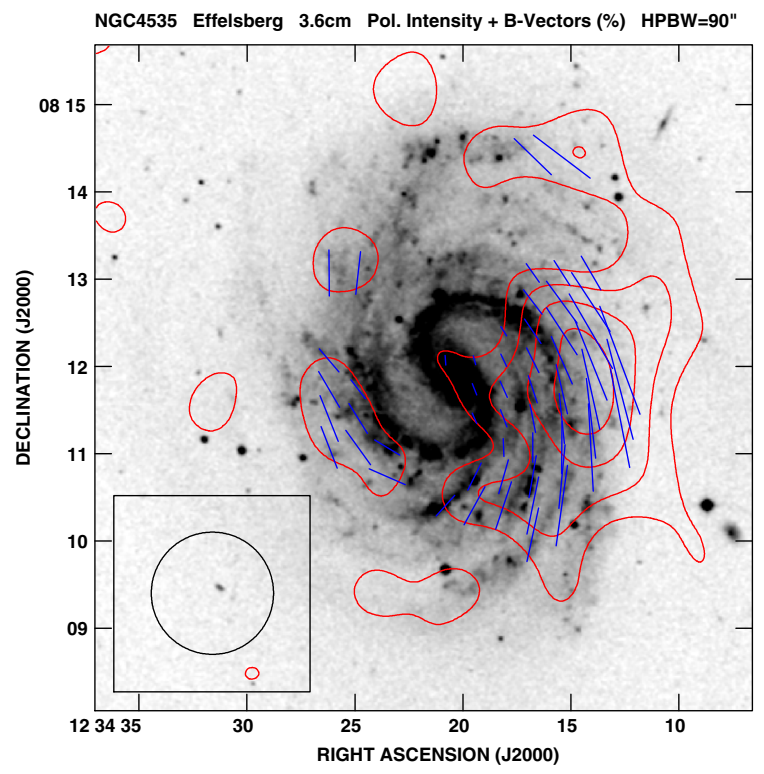


Fig. 23 Total radio intensity (contours) and B-vectors of the jets in NGC 4258, observed at $3.5 \mathrm{~cm}$ wavelength with the VLA at $14^{\prime \prime}$ resolution. The background $H \alpha$ image is from the Hoher List Observatory of the University of Bonn (from Krause and Löhr 2004)
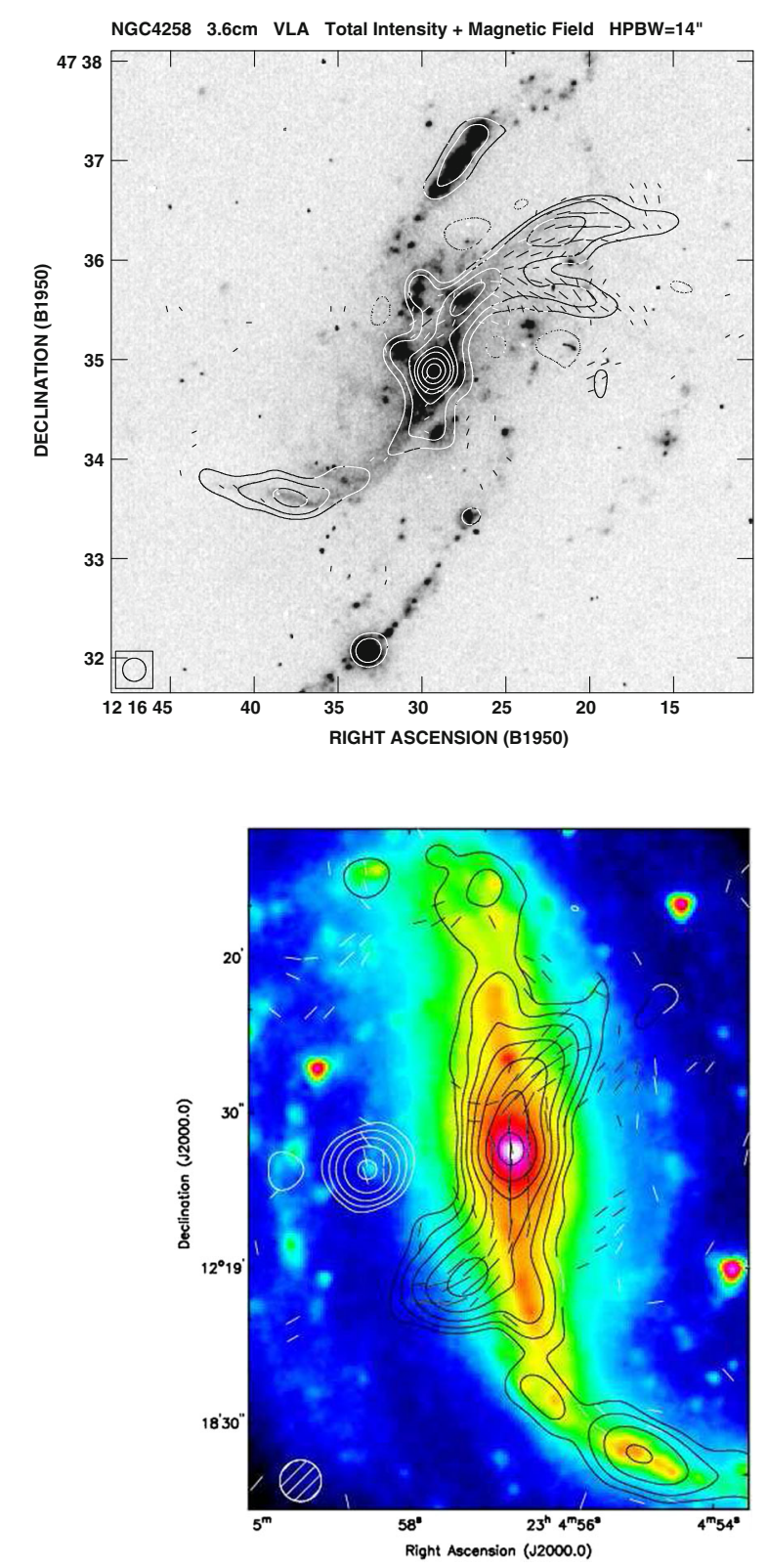

Fig. 24 Total radio intensity (contours) and B-vectors of the jets in NGC 7479, observed at $3.5 \mathrm{~cm}$ wavelength with the VLA at $8^{\prime \prime}$ resolution. The background shows a Spitzer/IRAC image at $3.6 \mu \mathrm{m}$ (NASA/JPL-Caltech/Seppo Laine) (from Laine and Beck 2008)

measurements, the large-scale regular magnetic field along the bar points towards the nucleus on both sides. Multiple reversals on scales of $1-2 \mathrm{kpc}$ are detected, probably occurring in the galaxy disk in front of the eastern jet by anisotropic fields in the shearing gas flow in the bar potential.

Highly polarized radio emission from kpc-size jets has also been detected in NGC 3079 (Cecil et al. 2001), with the field orientations perpendicular to the jet's axis 
and in the outflow lobes of the Circinus Galaxy (Elmouttie et al. 1995). Two members of the Virgo cluster, NGC 4388 and NGC 4438, have elongated radio features emerging from the active Seyfert-type nuclei and extending roughly perpendicular to the planes of the disks (Hummel and Saikia 1991). Detailed images including polarization are forthcoming as results of the CHANG-ES survey of edge-on galaxies (Irwin et al. 2012).

Jets in spiral galaxies may be more frequent than the available radio observations suggest. Future low-frequency observations will help to search for weak synchrotron emission from interface regions between the jets and the low-density halo gas.

\section{How does the Milky Way fit into the picture of nearby galaxies?}

Direct measurements of the Voyager 2 spacecraft in the heliosheath indicate that the surrounding interstellar magnetic field is $4-5 \mu \mathrm{G}$ strong and oriented at an angle of about $30^{\circ}$ from the Galactic plane (Opher et al. 2009), probably because the ISM field twists close to the heliosphere. Voyager 1 crossed into interstellar space in 2012 and measured a smooth increase in field strength to $5.62 \pm 0.01 \mu \mathrm{G}$ (Burlaga et al. 2013). This value is very close to those obtained with other methods (see below) and to those in nearby galaxies with a low star-formation rate (Sect. 4.1).

To deduce information about the strength and structure of the Galactic magnetic field, pulsars are ideal objects because their RMs provide field directions at many distances from the Sun (Noutsos 2012). Since most pulsars are concentrated along the Galactic plane, they sample the field in the disk. Combination of RM and DM data of pulsars (Eq. (11)), assuming uncorrelated fluctuations, gives an average strength of the local regular field along the line of sight of $2.1 \pm 0.3 \mu \mathrm{G}$ and about $4 \mu \mathrm{G}$ at $3 \mathrm{kpc}$ Galacto-centric radius (Han et al. 2006). These are upper limits in the case of correlated fluctuations (Beck et al. 2003), while lower limits in the case of anticorrelated fluctuations or field reversals along the line of sight.

From the dispersion of pulsar RMs, the Galactic magnetic field was found to have a significant turbulent component with a mean strength of 5-6 $\mu \mathrm{G}$ (Rand and Kulkarni 1989; Ohno and Shibata 1993; Han et al. 2004). Magnetic turbulence occurs over a large spectrum of scales, with the largest scale determined from pulsar RMs of $l_{\text {turb }} \simeq 55 \mathrm{pc}$ (Rand and Kulkarni 1989) or $l_{\text {turb }} \simeq 10-100 \mathrm{pc}$ (Ohno and Shibata 1993). These values are consistent with the size of turbulent cells of $d \simeq 50$ pc estimated from beam depolarization and Faraday depolarization in external galaxies (Sects. 3.5, 3.7). However, turbulence scales of only a few parsecs in spiral arms were derived from RM structure functions of polarized background sources (Haverkorn et al. 2008).

Modelling the surveys of the total synchrotron and $\gamma$-ray emission from the Milky Way yield field strengths near the Sun of about $5 \mu \mathrm{G}$ of the isotropic turbulent field, about $2 \mu \mathrm{G}$ of the anisotropic turbulent field and about $2 \mu \mathrm{G}$ of the regular field (Orlando and Strong 2013), adding up to a total field strength of about $6 \mu \mathrm{G}$. This is in excellent agreement with the Voyager and pulsar RM data (see above) and the Zeeman splitting data of low-density gas clouds (Crutcher et al. 2010). In the inner Galaxy the total field strength is about $10 \mu \mathrm{G}$. In the synchrotron filaments near the Galactic Centre the total field strength is about $100 \mu \mathrm{G}$ (Crocker et al. 2010). 


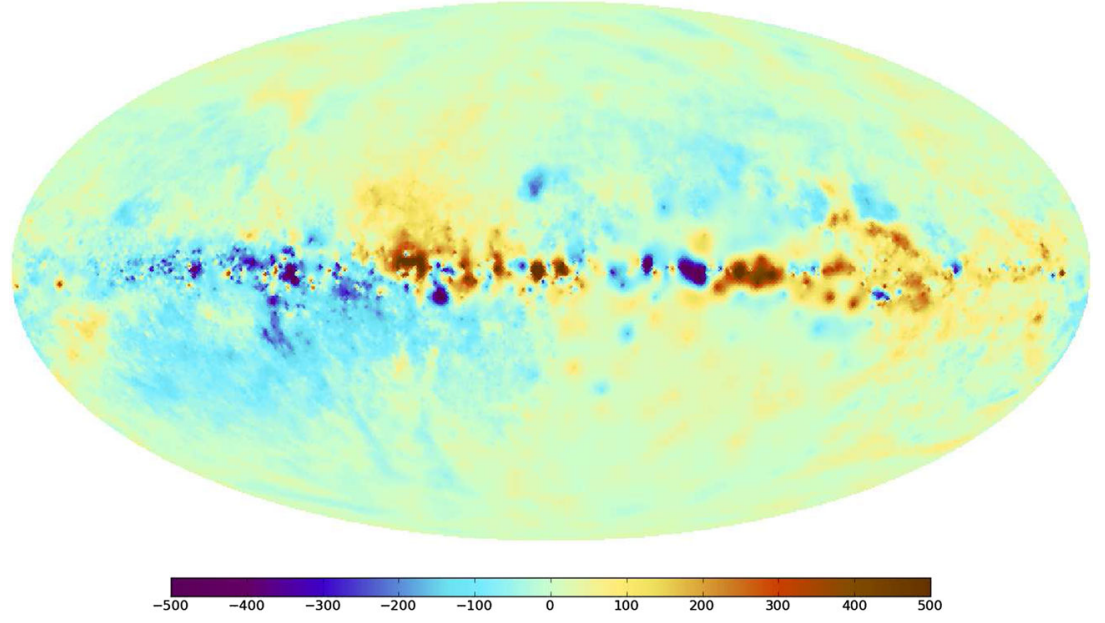

Fig. 25 All-sky map of rotation measures in the Milky Way, constructed from the RM data of about 40000 polarized extragalactic sources from the VLA NVSS survey (Taylor et al. 2009) and other catalogs. Red positive RM, blue negative RM (from Oppermann et al. 2012)

Optical polarization data of about 5500 selected stars in the Milky Way yielded the orientation of the large-scale magnetic field near the Sun (Fosalba et al. 2002), which is mostly parallel to the Galactic plane and oriented along the local spiral arm.

The all-sky maps of polarized synchrotron emission at $1.4 \mathrm{GHz}$ from the Milky Way from DRAO and Villa Elisa and at $22.8 \mathrm{GHz}$ from WMAP, and the Effelsberg RM survey of polarized extragalactic sources, were used to model the regular Galactic field (Sun et al. 2008; Sun and Reich 2010). One large-scale field reversal is required at about $1-2 \mathrm{kpc}$ from the Sun towards the Milky Way's centre, consistent with pulsar data.

RM data from pulsars and extragalactic radio sources (Fig. 25) were used to model the Galactic magnetic field (Nota and Katgert 2010; Van Eck et al. 2011; Jansson and Farrar 2012). A large-scale magnetic field reversal appears to be present between the Scutum-Crux-Sagittarius arm and the Carina-Orion arm (Fig. 26). As distances to most pulsars are uncertain, this result should be taken with some caution. The overall structure of the regular field in the disk of the Milky Way is still uncertain (Noutsos 2009). A larger sample of pulsar RM data and improved distance measurements to pulsars are needed. A satisfying explanation for the large-scale reversal in the Milky Way is still lacking. So far no similar reversals have been detected in external galaxies (Sect. 4.11). Possible reasons are as follows:

1. The reversal in the Milky Way may be restricted to a thin layer near to the plane and, therefore, hardly visible in the average RM data of external galaxies along the line of sight.

2. The reversal in the Milky Way may be of limited azimuthal extent and difficult to observe in external galaxies with present-day telescopes. 
Fig. 26 Model of the magnetic field in the Milky Way (vectors), derived from Faraday rotation measures of pulsars and extragalactic sources. Generally accepted results are indicated by yellow vectors, while white vectors are not fully confirmed. The background image shows the distribution of ionized gas delineating the spiral arms (from Jo-Anne Brown, Calgary)

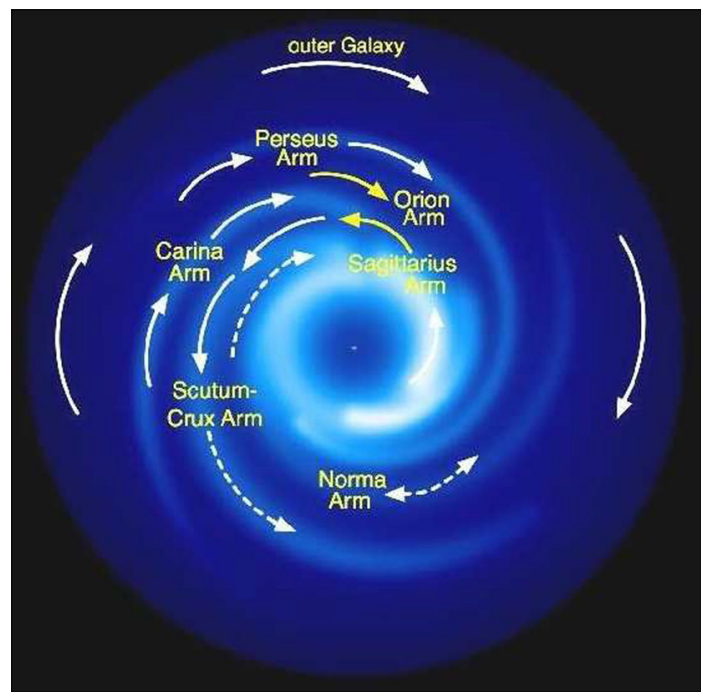

3. The reversal in the Milky Way may be part of a disturbed field structure, e.g. due to interaction with the Magellanic clouds, or a relic from seed fields (Moss et al. 2012).

Even less is known about the halo field of the Milky Way. The vertical full equivalent thickness of the synchrotron emission from the Milky Way is about $3 \mathrm{kpc}$ near the Sun (Beuermann et al. 1985), scaled to a distance to the Galactic centre of $8.5 \mathrm{kpc}$, yielding an exponential scale height of $H_{\text {syn }} \simeq 1.5 \mathrm{kpc}$ and at least $6 \mathrm{kpc}$ of the total field, similar to that in external galaxies (Sect. 4.13).

The signs of RMs of extragalactic sources and of pulsars at Galactic longitudes $l=90^{\circ}-270^{\circ}$ are mostly the same above and below the plane (Fig. 25): the magnetic field in the local disk is symmetric, while the RM signs towards the inner Galaxy $\left(l=270^{\circ}-90^{\circ}\right)$ are opposite above and below the plane. This could be assigned to an odd-symmetry (antisymmetric) halo field (Sun and Reich 2010). However, according to RM data from extragalactic sources, the local regular Galactic field has no significant vertical component towards the northern Galactic pole and only a weak vertical component of $B_{z} \simeq 0.3 \mu \mathrm{G}$ towards the south (Mao et al. 2010). This is consistent neither with an odd-symmetry nor with an even-symmetry halo field as found in several external galaxies (Sect. 4.7). The halo field of the Milky Way has a more complicated structure than that predicted by dynamo models. Modelling the diffuse polarized emission and RMs gave evidence for an X-shaped vertical field component (Jansson and Farrar 2012), similar to that in external galaxies (Sect. 4.13).

In summary, strength, spiral pattern and vertical extent of the Galactic magnetic field are similar to those in nearby spiral galaxies. Two major differences still need to be understood: the large-scale field reversal(s) and the antisymmetric pattern of the halo field, both of which are not observed in external galaxies. Either our Milky Way is special, or the different observational methods deliver apparently incompatible results. 
Table 3 General field structures in nearby galaxies

\begin{tabular}{lll}
\hline Galaxy type & Magnetic field structure & Regular field \\
\hline Sc with strong density wave & $\begin{array}{c}\text { Spiral at inner arm edge and in interarm } \\
\text { regions, turbulent in arms }\end{array}$ & Strong, fluctuating \\
$\begin{array}{l}\text { Sb or Sc with weak or } \\
\text { moderate density wave }\end{array}$ & $\begin{array}{c}\text { Spiral in interarm regions, turbulent }+ \\
\text { ordered in arms }\end{array}$ & Moderate, large-scale \\
Barred Sc & Ordered + turbulent along bar, spiral outside & Moderate \\
Sa & bar & Detected \\
S0 & Ordered + turbulent & Not detected \\
Interacting spiral & Not detected & Weak \\
Spiral with nuclear jets & Ordered, asymmetric & $?$ \\
Flocculent Sc or Sd & Ordered along jet & Weak \\
Irregular & Spiral + turbulent in disk & Weak \\
Starburst dwarf & Turbulent in star-forming regions + & Not detected \\
Spheroidal dwarf & segments of ordered field & Not detected \\
E without active nucleus & Turbulent in star-forming regions & Not detected \\
\hline
\end{tabular}

\section{Summary and open questions}

Table 3 gives a concise description of the general structures of ordered magnetic fields in various galaxy types as observed from radio polarization observations. For most galaxy types only a few cases were observed so far:"Turbulent" stands for "isotropic turbulent or tangled"; "ordered" for "anisotropic turbulent" or "regular"; "Regular field" refers to additional measurements of Faraday rotation.

For a list of radio polarization observations of galaxies, see Beck and Wielebinski (2013) and updated versions on arXiv. Radio images of total intensity and polarization are compiled on http://www.mpifr-bonn.mpg.de/atlasmag.

The open questions raised in this review may inspire future work:

\section{Morphology}

- How far do turbulent and regular magnetic fields extend radially in the disk and vertically above the disk?

- How well are magnetic pitch angles aligned with the orientations of gaseous spiral arms?

- How do magnetic pitch angles vary radially in the disk?

- Do dominating bisymmetric spiral patterns (azimuthal mode $m=1$ ) of the regular field exist in galaxies?

- Do azimuthal modes $m>2$ exist and how strong are these?

- Which galaxy types host magnetic arms between gaseous spiral arms?

- How common are field loops generated by the Parker instability?

- Do regular fields in galaxies have large-scale reversals? 
- Are the X-shaped fields in galaxy halos related to outflows (anisotropic turbulent fields) or do they show large-scale patterns in Faraday rotation (regular fields)?

2. Origin and amplification

- What was the main source of seed fields needed for the amplification of galactic fields in young galaxies?

- Are small-scale fields generated by the small-scale dynamo or by tangling of regular fields?

- What is the origin of magnetic arms between gaseous spiral arms?

- What determines the efficiency and saturation level of the $\alpha-\Omega$ dynamo?

- Is the mean helicity of the large-scale magnetic fields in galaxy disks non-zero, as predicted by $\alpha-\Omega$ dynamo action?

- Are small-scale fields with non-zero mean helicity expelled from the disk to keep the $\alpha-\Omega$ dynamo alive?

3. Dynamical importance

- Where is energy equipartition between total magnetic field and total cosmic rays valid and where does it fail?

- What is the dominant propagation mechanism of CREs in galaxy disks and halos and how does this depend on magnetic field strength and structure?

- How do magnetic fields interact with spiral density waves?

- How does the relation between total magnetic field strength and gas density vary between galaxies and within galaxies?

- How do magnetic fields modify the star-formation rate and the star-formation efficiency?

- Is amplification of small-scale fields in star-forming regions the reason for the radio-IR correlation?

- Is the magnetic energy density larger than that of gas turbulence in the outer parts of galaxies?

- Can magnetic fields affect the general rotation of gas?

- Can galactic outflows magnetize the intergalactic medium?

\section{Outlook}

Future high-resolution, high-sensitivity observations at high radio frequencies with the Jansky Very Large Array (VLA) and the planned Square Kilometre Array (SKA, see below), combined with high-resolution $\mathrm{CO}$ observations with the Atacama Large Millimeter Array (ALMA), will directly map the detailed field structure and the interaction with the molecular gas in external galaxies (Beck et al. 2015). Images of dust polarization of nearby galaxies in the submm range with ALMA will soon become possible. Another exciting prospect is extragalactic starlight and infrared polarimetry with the European Extremely Large Telescope (E-ELT) (Strassmeier and Ilyin 2009).

Construction of the first phase of the SKA is planned to start in 2018. The lowfrequency radio telescopes Low Frequency Array (LOFAR) (Haarlem et al. 2013) and the Murchison Widefield Array (MWA) (Tingay et al. 2013) are suitable instruments to search for extended synchrotron radiation in outer galaxy disks and halos and the 


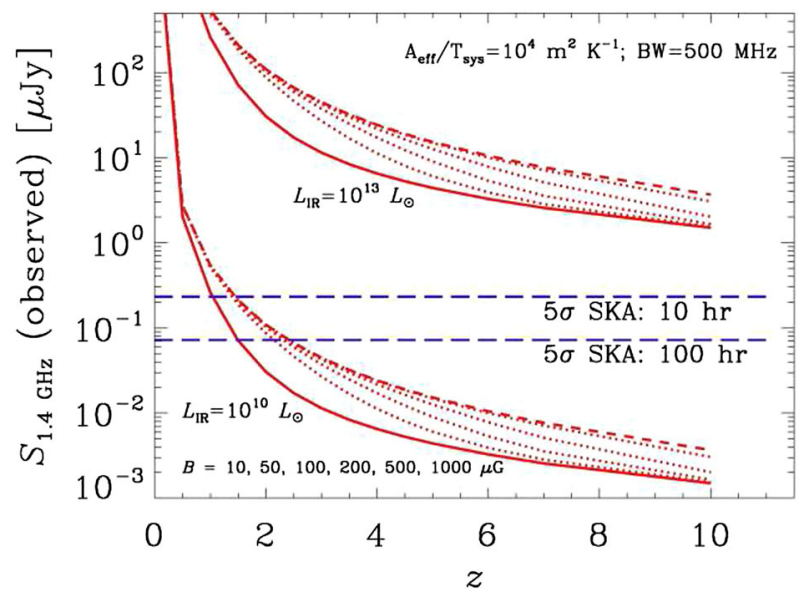

Fig. 27 Total synchrotron emission at $20 \mathrm{~cm}$ wavelength as a function of redshift $z$, total magnetic field strength $B$ and the $5 \sigma$ detection limits for 10 and $100 \mathrm{~h}$ integration time with the full SKA (from Murphy 2009 and priv. comm.)

transition region to intergalactic space. Several galaxies have already been observed with LOFAR (Beck et al. 2013; Mulcahy et al. 2014). Low frequencies are also suited to search for small Faraday rotation measures from weak fields in the local ISM of the Milky Way, in the ISM of external galaxies and in intergalactic space, if Faraday depolarization is small.

Small-scale and large-scale magnetic fields may exist in S0 and elliptical galaxies without star formation and without active nucleus because turbulence can be generated by the magneto-rotational instability (MRI) (Sellwood and Balbus 1999) or may result from star formation activity in the past. The detection may become possible with the SKA via deep imaging or RM grids of background sources.

The detection of strong radio emission in distant galaxies (which is at least partly of synchrotron origin) demonstrates that strong magnetic fields existed already in young galaxies with strengths of several $100 \mu \mathrm{G}$ (Murphy 2009). Total synchrotron emission can be detected with the SKA out to even larger redshifts in starburst galaxies, depending on luminosity and magnetic field strength (Fig. 27). As the amplification of turbulent fields is fast and efficient (Sect. 2) and the star-formation rates were high at high redshifts, the total fields can be expected to be very strong already in young galaxies. Then the energy range of the electrons emitting at a fixed observation frequency shifts to low energies (Eq. 3), where ionization and bremsstrahlung losses may become dominant so that there must be a maximum redshift until synchrotron emission is detectable. Its measurement with the SKA will constrain models of the evolution of magnetic fields in young galaxies (Schleicher and Beck 2013).

Nearby galaxies seen edge-on generally show an ordered, disk-parallel field near the disk plane (Dumke et al. 1995). As a result, polarized emission can also be detected from distant, unresolved galaxies if the inclination is larger than about $20^{\circ}$ (Stil et al. 2009). This opens a new method to search for ordered fields in distant galaxies with help of deep surveys. 
If polarized emission from galaxies is too weak to be detected, the method of $R M$ grids towards background QSOs can still be applied. Here, the distance limit is given by the polarized flux of the background QSO, which can be much higher than that of an intervening galaxy that is identified optically by its $\mathrm{Mg}$ II absorption system. With this method, significant regular fields of several $\mu \mathrm{G}$ strength were detected in distant galaxies (Bernet et al. 2008, 2013; Farnes et al. 2014). Mean-field solutions of the $\alpha-\Omega$ dynamo theory predict patches of regular fields observable as RMs in evolving regular fields already at $z \leq 3$, with increasing coherence scale, until fully developed regular fields are formed at $z \simeq 1-1.5$ (Arshakian et al. 2009; Rodrigues et al. 2015). Note that RM values measured today have been reduced due to redshift by the dilution factor of $(1+z)^{-2}$ so that observations at longer wavelengths are needed to detect RMs from regular fields at high redshifts.

Faraday rotation in the direction of polarized lobes of radio galaxies allows to determine the strength and pattern of a regular field in an intervening galaxy (Kronberg et al. 1992; Irwin et al. 2013). RM data indicate that ionized gas may extend to several $10 \mathrm{kpc}$ distance from galaxies (Bernet et al. 2013). A reliable model for the field structure in and around galaxies needs RM values from a large number of polarized background sources and hence large sensitivity and high survey speed.

The SKA “Cosmic Magnetism” Key Science Project plans to observe a polarization survey over the entire accessible sky with the SKA-MID Band 2 (around $20 \mathrm{~cm}$ wavelength) (Johnston-Hollitt et al. 2015). Within $1 \mathrm{~h}$ integration per field this will allow the detection of about 10 million discrete extragalactic sources and the measurement of their RMs, about 300 RMs per square degree or a mean spacing between sources of about $3^{\prime}$.

Deep polarization observations with the SKA centred on a sample of nearby galaxies will allow studies of the interaction between magnetic fields and the ISM gas (Beck et al. 2015). The detailed reconstruction of the 3D magnetic field structure will become possible with help of Faraday tomography (Heald et al. 2015) or a dense grid of RM values from background sources (Stepanov et al. 2008). More than 1000 RM values can be expected in the area of M 31. Applying this method to more distant galaxies, simple patterns of regular fields can be recognized out to distances of about $100 \mathrm{Mpc}$ (Stepanov et al. 2008).

\section{Exciting prospects!}

Acknowledgements The author would like to thank all colleagues who have pursued the studies of magnetic fields in the Milky Way and in galaxies over the past 40 years, especially Richard Wielebinski, Elly M. Berkhuijsen, Marita Krause, Patricia Reich, Wolfgang Reich, Sui Ann Mao and Aritra Basu at MPIfR, Andrew Fletcher and Anvar Shukurov at Newcastle University, Chris Chyży and Marek Urbanik at Kraków University, Dmitry Sokoloff at Moscow University, Peter Frick and Rodion Stepanov at ICMM Perm, RalfJürgen Dettmar at Bochum University, Detlef Elstner at AIP Potsdam, Katia Ferrière at Toulouse University and Fatemeh Tabatabaei at IAC La Laguna. Elly M. Berkhuijsen and Aritra Basu are acknowledged for careful reading of the manuscript and useful comments. Support from DFG Research Unit FOR1254 is acknowledged.

Open Access This article is distributed under the terms of the Creative Commons Attribution 4.0 International License (http://creativecommons.org/licenses/by/4.0/), which permits unrestricted use, distribution, and reproduction in any medium, provided you give appropriate credit to the original author(s) and the source, provide a link to the Creative Commons license, and indicate if changes were made. 


\section{References}

Aab A, Abreu P, Aglietta M et al (2015) Searches for anisotropies in the arrival directions of the highest energy cosmic rays detected by the Pierre Auger observatory. Astrophys J 804:15

Adebahr B, Krause M, Klein U et al (2013) M 82-a radio continuum and polarisation study. I. Data reduction and cosmic ray propagation. Astron Astrophys 555:A23

Arbutina B, Urošević D, Andjelić MM, Pavlović MZ, Vukotić B (2012) Modified equipartition calculation for supernova remnants. Astrophys J 746:79

Arshakian TG, Beck R (2011) Optimum frequency band for radio polarization observations. Mon Not R Astron Soc 418:2336

Arshakian TG, Beck R, Krause M, Sokoloff D (2009) Evolution of magnetic fields in galaxies and future observational tests with the Square Kilometre Array. Astron Astrophys 494:21

Bagetakos I, Brinks E, Walter F et al (2011) The fine-scale structure of the neutral interstellar medium in nearby galaxies. AJ 141:23

Balbus SA, Hawley JF (1998) Instability, turbulence, and enhanced transport in accretion disks. Rev Mod Phys 70:1

Basu A, Roy S (2013) Magnetic fields in nearby normal galaxies: energy equipartition. Mon Not R Astron Soc 433:1675

Basu A, Roy S, Mitra D (2012) Low-frequency radio-FIR correlation in normal galaxies at $1 \mathrm{kpc}$ scales. Astrophys J 756:141

Basu A, Wadadekar Y, Beelen A et al (2015) Radio-far-infrared correlation in "Blue Cloud" galaxies with $0<z<1.2$. Astrophys J 803:51

Battaner E, Florido E (2007) Are rotation curves in NGC 6946 and the Milky Way magnetically supported? Astron Nachr 328:92

Beck R (2007) Magnetism in the spiral galaxy NGC 6946: magnetic arms, depolarization rings, dynamo modes, and helical fields. Astron Astrophys 470:539

Beck R (2015) Magnetic fields in the nearby spiral galaxy IC 342: a multi-frequency radio polarization study. Astron Astrophys 578:A93

Beck R, Krause M (2005) Revised equipartition and minimum energy formula for magnetic field strength estimates from radio synchrotron observations. Astron Nachr 326:414

Beck R, Wielebinski R (2013) Magnetic fields in galaxies, in planets, stars and stellar systems. In: Oswalt TD, Gilmore G (eds) Galactic structure and stellar populations, vol 5. Springer, New York, p 641

Beck R, Loiseau N, Hummel E et al (1989) High-resolution polarization observations of M31. I-structure of the magnetic field in the southwestern arm. Astron Astrophys 222:58

Beck R, Poezd AD, Shukurov A, Sokoloff DD (1994) Dynamos in evolving galaxies. Astron Astrophys 289:94

Beck R, Brandenburg A, Moss D, Shukurov A, Sokoloff D (1996) Galactic magnetism: recent developments and perspectives. Ann Rev Astron Astrophys 34:155

Beck R, Shukurov A, Sokoloff D, Wielebinski R (2003) Systematic bias in interstellar magnetic field estimates. Astron Astrophys 411:99

Beck R, Fletcher A, Shukurov A et al (2005) Magnetic fields in barred galaxies. IV. NGC 1097 and NGC 1365. Astron Astrophys 444:739

Beck AM, Lesch H, Dolag K et al (2012a) Origin of strong magnetic fields in Milky Way-like galactic haloes. Mon Not R Astron Soc 422:2152

Beck R, Frick P, Stepanov R, Sokoloff D (2012b) Recognizing magnetic structures by present and future radio telescopes with Faraday rotation measure synthesis. Astron Astrophys 543:A113

Beck R, Anderson J, Heald G et al (2013) The LOFAR view of cosmic magnetism. Astron Nachr 334:548

Beck R, Bomans D, Colafrancesco S et al (2015) Structure, dynamical impact and origin of magnetic fields in nearby galaxies in the SKA era. In: Advancing astrophysics with the Square Kilometre Array (AASKA14), 94

Bell AR (1978) The acceleration of cosmic rays in shock fronts. II. Mon Not R Astron Soc 182:443

Bell EF (2003) Estimating star formation rates from infrared and radio luminosities: the origin of the radio-infrared correlation. Astrophys J 586:794

Bell MR, Junklewitz H, Enßlin TA (2011) Faraday caustics. Singularities in the Faraday spectrum and their utility as probes of magnetic field properties. Astron Astrophys 535:A85

Berkhuijsen EM, Horellou C, Krause M et al (1997) Magnetic fields in the disk and halo of M 51. Astron Astrophys 318:700 
Berkhuijsen EM, Beck R, Hoernes P (2003) The polarized disk in M 31 at lambda $6 \mathrm{~cm}$. Astron Astrophys 398:937

Berkhuijsen EM, Mitra D, Müller P (2006) Filling factors and scale heights of the diffuse ionized gas in the Milky Way. Astron Nachr 327:82

Berkhuijsen EM, Beck R, Tabatabaei FS (2013) How cosmic ray electron propagation affects radio-farinfrared correlations in M 31 and M 33. Mon Not R Astron Soc 435:1598

Bernet ML, Miniati F, Lilly SJ, Kronberg PP, Dessauges-Zavadsky M (2008) Strong magnetic fields in normal galaxies at high redshift. Nature 454:302

Bernet ML, Miniati F, Lilly SJ (2012) The interpretation of rotation measures in the presence of inhomogeneous foreground screens. Astrophys J 761:144

Bernet ML, Miniati F, Lilly SJ (2013) The extent of magnetic fields around galaxies out to z $\sim 1$. Astrophys J Lett 772:L28

Bertone S, Vogt C, Enßlin T (2006) Magnetic field seeding by galactic winds. Mon Not R Astron Soc $370: 319$

Beuermann K, Kanbach G, Berkhuijsen EM (1985) Radio structure of the galaxy - thick disk and thin disk at $408 \mathrm{MHz}$. Astron Astrophys 153:17

Bigiel F, Leroy A, Walter F et al (2008) The star formation law in nearby galaxies on sub-kpc scales. AJ 136:2846

Birnboim Y, Balberg S, Teyssier R (2015) Galaxy evolution: modelling the role of non-thermal pressure in the interstellar medium. Mon Not R Astron Soc 447:3678

Bisnovatyi-Kogan GS, Ruzmaikin AA, Syunyaev RA (1973) Star contraction and magnetic-field generation in protogalaxies. Sov Astron 17:137

Brandenburg A, Subramanian K (2005) Astrophysical magnetic fields and nonlinear dynamo theory. Phys Rep 417:1

Brandenburg A, Stepanov R (2014) Faraday signature of magnetic helicity from reduced depolarization. Astrophys J 786:91

Braun R, Heald G, Beck R (2010) The Westerbork SINGS survey. III. Global magnetic field topology. Astron Astrophys 514:A42

Brentjens MA, de Bruyn AG (2005) Faraday rotation measure synthesis. Astron Astrophys 441:1217

Broderick AE, Chang P, Pfrommer C (2012) The cosmological impact of luminous TeV blazars. I. Implications of plasma instabilities for the intergalactic magnetic field and extragalactic gamma-ray background. Astrophys J 752:22

Burlaga LF, Ness NF, Gurnett DA, Kurth WS (2013) Evidence for a shock in interstellar plasma: Voyager 1. Astrophys J Lett 778:L3

Burn BJ (1966) On the depolarization of discrete radio sources by Faraday dispersion. Mon Not R Astron Soc 133:67

Buta RJ, McCall ML (1999) The IC 342/Maffei group revealed. Astrophys J Suppl 124:33

Calzetti D, Wu S-Y, Hong S et al (2010) The calibration of monochromatic far-infrared star formation rate indicators. Astrophys J 714:1256

Cecil G, Bland-Hawthorn J, Veilleux S, Filippenko AV (2001) Jet- and wind-driven ionized outflows in the superbubble and star-forming disk of NGC 3079. Astrophys J 555:338

Chamandy L, Subramanian K, Shukurov A (2013a) Galactic spiral patterns and dynamo action-I. A new twist on magnetic arms. Mon Not R Astron Soc 428:3569

Chamandy L, Subramanian K, Shukurov A (2013b) Galactic spiral patterns and dynamo action-II. Asymptotic solutions. Mon Not R Astron Soc 433:3274

Chamandy L, Shukurov A, Subramanian K (2015) Magnetic spiral arms and galactic outflows. Mon Not R Astron Soc 446:L6

Chandrasekhar S, Fermi E (1953) Magnetic fields in spiral arms. Astrophys J 118:113

Cho J, Lazarian A (2005) Grain alignment by radiation in dark clouds and cores. Astrophys J 631:361

Chyży KT (2008) Magnetic fields and gas in the cluster-influenced spiral galaxy NGC 4254. II. Structures of magnetic fields. Astron Astrophys 482:755

Chyży KT, Beck R (2004) Magnetic fields in merging spirals-the Antennae. Astron Astrophys 417:541

Chyży KT, Buta RJ (2008) Discovery of a strong spiral magnetic field crossing the inner pseudoring of NGC 4736. Astrophys J Lett 677:L17

Chyży KT, Beck R, Kohle S, Klein U, Urbanik M (2000) Regular magnetic fields in the dwarf irregular galaxy NGC 4449. Astron Astrophys 355:128 
Chyży KT, Weżgowiec M, Beck R, Bomans DJ (2011) Magnetic fields in local group dwarf irregulars. Astron Astrophys 529:A94

Cox DP (2005) The three-phase interstellar medium revisited. Ann Rev Astron Astrophys 43:337

Crocker RM, Jones DI, Melia F, Ott J, Protheroe RJ (2010) A lower limit of 50 microgauss for the magnetic field near the Galactic Centre. Nature 463:65

Crosthwaite LP, Turner JL, Hurt RL et al (2001) CO and neutral gas in the disk of the spiral galaxy IC 342. AJ 122:797

Crutcher RM, Wandelt B, Heiles C, Falgarone E, Troland TH (2010) Magnetic fields in interstellar clouds from Zeeman observations: inference of total field strengths by Bayesian analysis. Astrophys J 725:466

de Avillez MA, Breitschwerdt D (2005) Global dynamical evolution of the ISM in star forming galaxies. I. High resolution 3D simulations: effect of the magnetic field. Astron Astrophys 436:585

Dobbs C, Baba J (2014) Dawes review 4: spiral structures in disc galaxies. Publ Astron Soc Aust 31:35

Dolag K, Kachelriess M, Ostapchenko S, Tomàs R (2011) Lower limit on the strength and filling factor of extragalactic magnetic fields. Astrophys J Lett 727:L4

Drzazga RT, Chyży KT, Jurusik W, Wiórkiewicz K (2011) Magnetic field evolution in interacting galaxies. Astron Astrophys 533:A22

Dumas G, Schinnerer E, Tabatabaei FS et al (2011) The local radio-IR relation in M51. AJ 141:41

Dumke M, Krause M, Wielebinski R, Klein U (1995) Polarized radio emission at $2.8 \mathrm{~cm}$ from a selected sample of edge-on galaxies. Astron Astrophys 302:691

Durrer R, Neronov A (2013) Cosmological magnetic fields: their generation, evolution and observation. Astron Astrophys Rev 21:62

Ehle M, Beck R (1993) Ionized gas and intrinsic magnetic fields in the spiral galaxy NGC6946. Astron Astrophys 273:45

Ehle M, Beck R, Haynes RF et al (1996) Magnetic fields and hot gas in the spiral galaxy NGC 1566 as derived from ATCA radio polarization and ROSAT X-ray observations. Astron Astrophys 306:73

Ellis RS, Axon DJ (1978) Stellar polarization and the structure of the magnetic field of our Galaxy. Astrophys Space Sci 54:425

Elmouttie E, Haynes RF, Jones KL et al (1995) The polarized radio lobes of the Circinus galaxy. Mon Not R Astron Soc 275:L53

Elstner D, Beck R, Gressel O (2014) Do magnetic fields influence gas rotation in galaxies? Astron Astrophys 568:A104

Farnes JS, O’Sullivan SP, Corrigan ME, Gaensler BM (2014) Faraday rotation from magnesium II absorbers toward polarized background radio sources. Astrophys J 795:63

Farnsworth D, Rudnick L, Brown S (2011) Integrated polarization of sources at $\lambda 1 \mathrm{~m}$ and new rotation measure ambiguities. AJ 141:191

Fathi K, Toonen S, Falcón-Barroso J et al (2007) Quantifying resonant structure in NGC 6946 from twodimensional kinematics. Astrophys J Lett 667:L137

Fendt C, Beck R, Neininger N (1998) Spiral pattern in the optical polarization of NGC 6946. Astron Astrophys 335:123

Ferrière K (1996) Alpha-tensor and diffusivity tensor due to supernovae and superbubbles in the Galactic disk near the Sun. Astron Astrophys 310:438

Ferrière KM (2001) The interstellar environment of our Galaxy. Rev Mod Phys 73:1031

Fletcher A (2010) Magnetic fields in nearby galaxies. In: Kothes R, Landecker TL, Willis AG (eds) Astronomical Society of the Pacific Conference Series vol 438, p 197

Fletcher A, Berkhuijsen EM, Beck R, Shukurov A (2004) The magnetic field of M31 from multi-wavelength radio polarization observations. Astron Astrophys 414:53

Fletcher A, Beck R, Shukurov A, Berkhuijsen EM, Horellou C (2011) Magnetic fields and spiral arms in the galaxy M51. Mon Not R Astron Soc 412:2396

Foglizzo T, Tagger M (1995) The Parker-shearing instability in azimuthally magnetized discs. Astron Astrophys 301:293

Fosalba P, Lazarian A, Prunet S, Tauber JA (2002) Statistical properties of galactic starlight polarization. Astrophys J 564:762

Frick P, Beck R, Berkhuijsen EM, Patrickeyev I (2001) Scaling and correlation analysis of galactic images. Mon Not R Astron Soc 327:1145

Frick P, Sokoloff D, Stepanov R, Beck R (2011) Faraday rotation measure synthesis for magnetic fields of galaxies. Mon Not R Astron Soc 414:2540 
Frick P, Stepanov R, Beck R, et al (2016) Magnetic and gaseous spiral arms in M83. Astron Astrophys 585:A21

Gaensler BM, Haverkorn M, Staveley-Smith L et al (2005) The magnetic field of the Large Magellanic Cloud revealed through Faraday rotation. Science 307:1610

Gent FA, Shukurov A, Sarson GR, Fletcher A, Mantere MJ (2013) The supernova-regulated ISM-II. The mean magnetic field. Mon Not R Astron Soc 430:L40

Gießübel R (2012) Polarimetry of M31 from Megahertz to Gigahertz frequencies. PhD dissertation. Universität zu Köln, Cuvillier Verlag Göttingen. ISBN 978-3-95404-308-8

Gießübel R, Beck R (2014) The magnetic field structure of the central region in M 31. Astron Astrophys 571:A61

Gießübel R, Heald G, Beck R, Arshakian TG (2013) Polarized synchrotron radiation from the Andromeda galaxy M 31 and background sources at $350 \mathrm{MHz}$. Astron Astrophys 559:A27

Gissinger C, Fromang S, Dormy E (2009) Direct numerical simulations of the galactic dynamo in the kinematic growing phase. Mon Not R Astron Soc 394:L84

Goldreich P, Kylafis ND (1981) On mapping the magnetic field direction in molecular clouds by polarization measurements. Astrophys J Lett 243:L75

Golla G, Hummel E (1994) The intrinsic magnetic field orientation in NGC 4631. Astron Astrophys 284:777

Greaves JS, Holland WS, Jenness T, Hawarden TG (2000) Magnetic field surrounding the starburst nucleus of the galaxy M82 from polarized dust emission. Nature 404:732

Greenhill LJ, Jiang DR, Moran JM et al (1995) Detection of a subparsec diameter disk in the nucleus of NGC 4258. Astrophys J 440:619

Gressel O, Elstner D, Ziegler U (2013) Towards a hybrid dynamo model for the Milky Way. Astron Astrophys 560:A93

Han JL, Beck R, Ehle M, Haynes RF, Wielebinski R (1999) Magnetic fields in the spiral galaxy NGC 2997. Astron Astrophys 348:405

Han JL, Ferriere K, Manchester RN (2004) The spatial energy spectrum of magnetic fields in our Galaxy. Astrophys J 610:820

Han JL, Manchester RN, Lyne AG, Qiao GJ, van Straten W (2006) Pulsar rotation measures and the large-scale structure of the Galactic magnetic field. Astrophys J 642:868

Hanasz M, Otmianowska-Mazur K, Lesch H (2002) Topological evolution of Parker-unstable galactic magnetic fields under the influence of Coriolis force and magnetic reconnection. Astron Astrophys 386:347

Hanasz M, Wóltański D, Kowalik K (2009) Global galactic dynamo driven by cosmic rays and exploding magnetized stars. Astrophys J Lett 706:L155

Hanasz M, Lesch H, Naab T et al (2013) Cosmic rays can drive strong outflows from gas-rich high-redshift disk galaxies. Astrophys J Lett 777:L38

Hanayama H, Takahashi K, Kotake K et al (2005) Biermann mechanism in primordial supernova remnant and seed magnetic fields. Astrophys J 633:941

Harnett J, Ehle M, Fletcher A et al (2004) Magnetic fields in barred galaxies. III. The southern peculiar galaxy NGC 2442. Astron Astrophys 421:571

Haverkorn M, Brown JC, Gaensler BM, McClure-Griffiths NM (2008) The outer scale of turbulence in the magnetoionized Galactic interstellar medium. Astrophys J 680:362

Haynes RF, Klein U, Wayte SR et al (1991) A radio continuum study of the Magellanic Clouds. I-complete multi-frequency maps. Astron Astrophys 252:475

Heald G (2015) Synchrotron radiation and Faraday rotation. In: Lazarian A, de Gouveia Dal Pino EM, Melioli C (eds) Magnetic fields in diffuse media, Astrophysics and Space Science Library, vol 407, p 41

Heald G, Braun R, Edmonds R (2009) The Westerbork SINGS survey. II. Polarization, Faraday rotation, and magnetic fields. Astron Astrophys 503:409

Heald G, Beck R, de Blok WJG et al (2015) Magnetic field tomography in nearby galaxies with the Square Kilometre Array. In: Advancing astrophysics with the Square Kilometre Array (AASKA14), 106

Heesen V, Beck R, Krause M, Dettmar R-J (2009a) Cosmic rays and the magnetic field in the nearby starburst galaxy NGC 253. I. The distribution and transport of cosmic rays. Astron Astrophys 494:563

Heesen V, Krause M, Beck R, Dettmar R-J (2009b) Cosmic rays and the magnetic field in the nearby starburst galaxy NGC 253. II. The magnetic field structure. Astron Astrophys 506:1123

Heesen V, Beck R, Krause M, Dettmar R-J (2011a) Cosmic rays and the magnetic field in the nearby starburst galaxy NGC 253 III. Helical magnetic fields in the nuclear outflow. Astron Astrophys 535:A79 
Heesen V, Rau U, Rupen MP, Brinks E, Hunter DA (2011b) Deep radio continuum imaging of the dwarf irregular galaxy IC 10: tracing star formation and magnetic fields. Astrophys J Lett 739:L23

Heesen V, Brinks E, Leroy AK et al (2014) The radio continuum-star formation rate relation in WSRT SINGS galaxies. AJ 147:103

Heiles C, Crutcher R (2005) Magnetic fields in diffuse HI and molecular clouds. In: Wielebinski R, Beck $\mathrm{R}$ (eds) Cosmic magnetic fields. Lecture Notes in Physics, vol 664. Springer, Berlin, p 137

Helfer TT, Thornley MD, Regan MW et al (2003) The BIMA survey of nearby galaxies (BIMA SONG). II. The CO data. Astrophys J Suppl 145:259

Hildebrand RH, Kirby L, Dotson JL, Houde M, Vaillancourt JE (2009) Dispersion of magnetic fields in molecular clouds. I. Astrophys J 696:567

Hill AS, Joung MR, Mac Low M-M et al (2012) Vertical structure of a supernova-driven turbulent, magnetized interstellar medium. Astrophys J 750:104

Hoang T, Lazarian A (2008) Radiative torque alignment: essential physical processes. Mon Not R Astron Soc 388:117

Hoang T, Lazarian A (2014) Grain alignment by radiative torques in special conditions and implications. Mon Not R Astron Soc 438:680

Horellou C, Fletcher A (2014) Magnetic field tomography, helical magnetic fields and Faraday depolarization. Mon Not R Astron Soc 441:2049

Houde M, Vaillancourt JE, Hildebrand RH, Chitsazzadeh S, Kirby L (2009) Dispersion of magnetic fields in molecular clouds. II. Astrophys J 706:1504

Houde M, Rao R, Vaillancourt JE, Hildebrand RH (2011) Dispersion of magnetic fields in molecular clouds. III. Astrophys J 733:109

Houde M, Fletcher A, Beck R et al (2013) Characterizing magnetized turbulence in M51. Astrophys J 766:49

Hummel E, Saikia DJ (1991) The anomalous radio features in NGC 4388 and NGC 4438. Astron Astrophys 249:43

Ideguchi S, Tashiro Y, Akahori T, Takahashi K, Ryu D (2014) Faraday dispersion functions of galaxies. Astrophys J 792:51

Irwin J, Beck R, Benjamin RA et al (2012) Continuum halos in nearby galaxies: an EVLA survey (CHANGES). I. Introduction to the survey. AJ 144:43

Irwin J, Krause M, English J et al (2013) CHANG-ES. III. UGC 10288 - an edge-on galaxy with a background double-lobed radio source. AJ 146:164

Jansson R, Farrar GR (2012) A new model of the Galactic magnetic field. Astrophys J 757:14

Johnston-Hollitt M, Govoni F, Beck R et al (2015) Using SKA rotation measures to reveal the mysteries of the magnetised universe. In: Advancing astrophysics with the Square Kilometre Array (AASKA14), 92

Kepley AA, Mühle S, Everett J et al (2010) The role of the magnetic field in the interstellar medium of the post-starburst dwarf irregular galaxy NGC 1569. Astrophys J 712:536

Kim W-T, Stone JM (2012) Two-dimensional magnetohydrodynamic simulations of barred galaxies. Astrophys J 751:124

Kim W-T, Ostriker EC, Stone JM (2002) Three-dimensional simulations of Parker, magneto-Jeans, and swing instabilities in shearing galactic gas disks. Astrophys J 581:1080

Kim C-G, Kim, W-T, Ostriker, EC (2006) Interstellar turbulence driving by galactic spiral shocks. Astrophys J 649(1):L13

Kleeorin NI, Rogachevskii IV (1990) A new kind of magnetic buoyancy instability. In: Guyenne TD, Hunt JJ (eds) ESA special publication, vol 311, pp 21-23

Klein U, Fletcher A (2015) Galactic and intergalactic magnetic fields. Springer, Heidelberg

Knapik J, Soida M, Dettmar R-J, Beck R, Urbanik M (2000) Detection of spiral magnetic fields in two flocculent galaxies. Astron Astrophys 362:910

Kotarba H, Lesch H, Dolag K et al (2009) Magnetic field structure due to the global velocity field in spiral galaxies. Mon Not R Astron Soc 397:733

Kotarba H, Karl SJ, Naab T et al (2010) Simulating magnetic fields in the Antennae galaxies. Astrophys J 716:1438

Krause M (1990) Multi-frequency radio observations of spiral galaxies and their interpretation. In: Beck R, Wielebinski R, Kronberg PP (eds) IAU symposium, Galactic and intergalactic magnetic fields, vol 140, pp 187-196 
Krause M (2009) Magnetic fields and star formation in spiral galaxies. Rev Mex de Astron y Astrofis Conf Ser 36:25-29

Krause M (2014) Magnetic fields and halos in spiral galaxies. arXiv:1401.1317

Krause F, Beck R (1998) Symmetry and direction of seed magnetic fields in galaxies. Astron Astrophys 335:789

Krause M, Löhr A (2004) The magnetic field along the jets of NGC 4258. as deduced from high frequency radio observations. Astron Astrophys 420:115

Krause M, Beck R, Hummel E (1989) The magnetic field structures in two nearby spiral galaxies-part two- the bisymmetric spiral magnetic field in M81. Astron Astrophys 217:17

Krause M, Wielebinski R, Dumke M (2006) Radio polarization and sub-millimeter observations of the Sombrero galaxy (NGC 4594). Large-scale magnetic field configuration and dust emission. Astron Astrophys 448:133

Kronberg PP, Perry JJ, Zukowski ELH (1992) Discovery of extended Faraday rotation compatible with spiral structure in an intervening galaxy at $z=0.395$-new observations of PKS 1229-021. Astrophys J 387:528

Kulpa-Dybeł K, Otmianowska-Mazur K, Kulesza-Żydzik B et al (2011) Global simulations of the magnetic field evolution in barred galaxies under the influence of the cosmic-ray-driven dynamo. Astrophys $\mathrm{J}$ Lett 733:L18

Kulsrud RM, Cen R, Ostriker JP, Ryu D (1997) The protogalactic origin for cosmic magnetic fields. Astrophys J 480:481

Lacki BC, Beck R (2013) The equipartition magnetic field formula in starburst galaxies: accounting for pionic secondaries and strong energy losses. Mon Not R Astron Soc 430:3171

Lacki BC, Thompson TA, Quataert E (2010) The physics of the far-infrared-radio correlation. I. Calorimetry, conspiracy, and implications. Astrophys J 717:1

Laine S, Beck R (2008) Radio continuum jet in NGC 7479. Astrophys J 673:128

Lazar M, Schlickeiser R, Wielebinski R, Poedts S (2009) Cosmological effects of Weibel-type instabilities. Astrophys J 693:1133

Lerche I, Schlickeiser R (1982a) On the transport and propagation of relativistic electrons in galaxies. The effect of adiabatic deceleration in a galactic wind for the steady state case. Astron Astrophys 107:148

Lerche I, Schlickeiser R (1982b) Transport and propagation of cosmic rays in galaxies. II-the effect of a galactic wind on the mean lifetime and age distribution of non-decaying cosmic rays. Astron Astrophys 116:10

Li H-B, Henning T (2011) The alignment of molecular cloud magnetic fields with the spiral arms in M33. Nature 479:499

Liu F, Gao Y (2010) The radio continuum, far-infrared emission, and dense molecular gas in galaxies. Astrophys J 713:524

Lobo Gomes AL, Magalhães AM, Pereyra A, Rodrigues CV (2015) A new optical polarization catalog for the Small Magellanic Cloud: the magnetic field structure. Astrophys J 806:94

Lou Y-Q, Han JL, Fan Z (1999) Fast magnetohydrodynamic density waves in spiral galaxies. Mon Not R Astron Soc 308:L1

Machida M, Nakamura KE, Kudoh T et al (2013) Dynamo activities driven by magnetorotational instability and the Parker instability in galactic gaseous disks. Astrophys J 764:81

Mao SA, Gaensler BM, Stanimirović S et al (2008) A radio and optical polarization study of the magnetic field in the Small Magellanic Cloud. Astrophys J 688:1029

Mao SA, Gaensler BM, Haverkorn M et al (2010) A survey of extragalactic Faraday rotation at high Galactic latitude: the vertical magnetic field of the Milky Way toward the Galactic poles. Astrophys J 714:1170

Mao SA, McClure-Griffiths NM, Gaensler BM et al (2012) Magnetic field structure of the Large Magellanic Cloud from Faraday rotation measures of diffuse polarized emission. Astrophys J 759:25

Mao SA, Zweibel E, Fletcher A, Ott J, Tabatabaei F (2015) Properties of the magneto-ionic medium in the halo of M51 revealed by wide-band polarimetry. Astrophys J 800:92

Meidt SE, Rand RJ, Merrifield MR (2009) Uncovering the origins of spiral structure by measuring radial variation in pattern speeds. Astrophys J 702:277

Meier DS (2014) The nucleus of IC 342 as a potential twin of the Galactic center. In: Sjouwerman LO, Lang CC, Ott J (eds) IAU symposium, vol 303, pp 66-68

Mikhailov E, Kasparova A, Moss D et al (2014) Magnetic fields near the peripheries of galactic discs. Astron Astrophys 568:A66

Mora SC, Krause M (2013) Magnetic field structure and halo in NGC 4631. Astron Astrophys 560:A42 
Moss D (1998) The relation between magnetic and gas arms in spiral galaxies. Mon Not R Astron Soc 297:860

Moss D, Shukurov A, Sokoloff D (1998) Boundary effects and propagating, magnetic fronts in disc dynamos. Geophys Astrophys Fluid Dyn 89:285

Moss D, Sokoloff D, Beck R, Krause M (2010) Galactic winds and the symmetry properties of galactic magnetic fields. Astron Astrophys 512:A61

Moss D, Beck R, Sokoloff D et al (2013) The relation between magnetic and material arms in models for spiral galaxies. Astron Astrophys 556:A147

Moss D, Stepanov R, Arshakian TG et al (2012) Multiscale magnetic fields in spiral galaxies: evolution and reversals. Astron Astrophys 537:A68

Moss D, Sokoloff D, Beck R, Krause M (2014) Enhancement of magnetic fields arising from galactic encounters. Astron Astrophys 566:A40

Moss D, Stepanov R, Krause M, Beck R, Sokoloff D (2015) The formation of regular interarm magnetic fields in spiral galaxies. Astron Astrophys 578:A94

Mulcahy DD, Horneffer A, Beck R et al (2014) The nature of the low-frequency emission of M 51. First observations of a nearby galaxy with LOFAR. Astron Astrophys 568:A74

Murgia M, Helfer TT, Ekers R et al (2005) The molecular connection to the FIR-radio continuum correlation in galaxies. Astron Astrophys 437:389

Murphy EJ (2009) The far-infrared-radio correlation at high redshifts: physical considerations and prospects for the Square Kilometre Array. Astrophys J 706:482

Niklas S (1995) Eigenschaften von Spiralgalaxien im hochfrequenten Radiokontinuum. PhD thesis, University of Bonn

Niklas S, Beck R (1997) A new approach to the radio-far infrared correlation for non-calorimeter galaxies. Astron Astrophys 320:54

Nota T, Katgert P (2010) The large-scale magnetic field in the fourth Galactic quadrant. Astron Astrophys 513:A65

Noutsos A (2009) Measuring ISM fields using pulsars. In: Strassmeier KG, Kosovichev AG, Beckman JE (eds) IAU symposium, vol 259, pp 15-24

Noutsos A (2012) The magnetic field of the Milky Way from Faraday rotation of pulsars and extragalactic sources. Space Sci Rev 166:307

Ohno H, Shibata S (1993) The random magnetic field in the Galaxy. Mon Not R Astron Soc 262:953

Opher M, Bibi FA, Toth G et al (2009) A strong, highly-tilted interstellar magnetic field near the solar system. Nature 462:1036

Oppermann N, Junklewitz H, Robbers G et al (2012) An improved map of the Galactic Faraday sky. Astron Astrophys 542:A93

Orlando E, Strong A (2013) Galactic synchrotron emission with cosmic ray propagation models. Mon Not R Astron Soc 436:2127

Otmianowska-Mazur K, Elstner D, Soida M, Urbanik M (2002) Magnetic fields and radio polarization of barred galaxies. 3D dynamo simulations. Astron Astrophys 384:48

Pakmor R, Springel V (2013) Simulations of magnetic fields in isolated disc galaxies. Mon Not R Astron Soc 432:176

Pakmor R, Marinacci F, Springel V (2014) Magnetic fields in cosmological simulations of disk galaxies. Astrophys J Lett 783:L20

Patrikeev I, Fletcher A, Stepanov R et al (2006) Analysis of spiral arms using anisotropic wavelets: gas, dust and magnetic fields in M 51. Astron Astrophys 458:441

Pierini D, Popescu CC, Tuffs RJ, Völk HJ (2003) The far-infrared/radio correlation in the ISO era. The warm and cold far-infrared/radio correlations. Astron Astrophys 409:907

Planck Collaboration, Ade PAR, Aghanim N et al (2015a) Planck intermediate results. XXXV. Probing the role of the magnetic field in the formation of structure in molecular clouds. arXiv: 1502.04123

Planck Collaboration, Ade PAR, Aghanim N et al (2015b) Planck 2015 results. XIX. Constraints on primordial magnetic fields. arXiv: 1502.01594

Poedts S, Rogava AD (2002) Does spiral galaxy IC 342 exhibit shear induced wave transformations? Astron Astrophys 385:32

Price DJ, Bate MR (2008) The effect of magnetic fields on star cluster formation. Mon Not R Astron Soc $385: 1820$

Rand RJ, Kulkarni SR (1989) The local Galactic magnetic field. Astrophys J 343:760 
Rees MJ (2005) Magnetic fields in the early universe. In: Wielebinski R, Beck R (eds) Cosmic magnetic fields. Lecture Notes in Physics, vol 664. Springer, Berlin, p 1

Reuter H-P, Klein U, Lesch H, Wielebinski R, Kronberg PP (1994) The magnetic field in the halo of M 82. Polarized radio emission at lambda 6 and $3.6 \mathrm{~cm}$. Astron Astrophys 282:724

Rieder M, Teyssier R (2015) A small-scale dynamo in feedback-dominated galaxies as the origin of cosmic magnetic fields. I-the kinematic phase. arXiv:1506.00849

Robishaw T, Quataert E, Heiles C (2008) Extragalactic zeeman detections in OH megamasers. Astrophys J 680:981

Rodrigues LFS, Shukurov A, Fletcher A, Baugh CM (2015) Galactic magnetic fields and hierarchical galaxy formation. Mon Not R Astron Soc 450:3472

Rohde R, Beck R, Elstner D (1999) Magnetic arms in NGC 6946 generated by a turbulent dynamo. Astron Astrophys 350:423

Scarrott SM, Ward-Thompson D, Warren-Smith RF (1987) Evidence for a spiral magnetic field configuration in the galaxy M51. Mon Not R Astron Soc 224:299

Schinnerer E, Meidt SE, Pety J et al (2013) The PdBI Arcsecond Whirlpool Survey (PAWS). I. A cloudscale/multi-wavelength view of the interstellar medium in a grand-design spiral galaxy. Astrophys $\mathrm{J}$ 779:42

Schleicher DRG, Beck R (2013) A new interpretation of the far-infrared-radio correlation and the expected breakdown at high redshift. Astron Astrophys 556:A142

Schleicher DRG, Banerjee R, Sur S et al (2010) Small-scale dynamo action during the formation of the first stars and galaxies. I. The ideal MHD limit. Astron Astrophys 522:A115

Schlickeiser R (2012) Cosmic magnetization: from spontaneously emitted aperiodic turbulent to ordered equipartition fields. Phys Rev Lett 109:261101

Schlickeiser R, Felten T (2013) Strength of the spontaneously emitted collective aperiodic magnetic field fluctuations in the reionized early intergalactic medium. Astrophys J 778:39

Sellwood JA, Balbus SA (1999) Differential rotation and turbulence in extended HI disks. Astrophys J 511:660

Seymour N, Dwelly T, Moss D et al (2008) The star formation history of the Universe as revealed by deep radio observations. Mon Not $\mathrm{R}$ Astron Soc 386:1695

Shi Y, Helou G, Yan L et al (2011) Extended Schmidt law: role of existing stars in current star formation. Astrophys J 733:87

Shibata K, Matsumoto R (1991) Formation of giant molecular clouds and helical magnetic fields by the Parker instability. Nature 353:633

Shneider C, Haverkorn M, Fletcher A, Shukurov A (2014) Depolarization of synchrotron radiation in a multilayer magneto-ionic medium. Astron Astrophys 567:A82

Shukurov A (1998) Magnetic spiral arms in galaxies. Mon Not R Astron Soc 299:L21

Shukurov A (2005) Mesoscale magnetic structures in spiral galaxies. In: Wielebinski R, Beck R (eds) Cosmic magnetic fields. Lecture Notes in Physics, vol 664. Springer, Berlin, p 113

Shukurov A, Sokoloff D, Subramanian K, Brandenburg A (2006) Galactic dynamo and helicity losses through fountain flow. Astron Astrophys 448:L33

Siejkowski H, Otmianowska-Mazur K, Soida M, Bomans DJ, Hanasz M (2014) 3D global simulations of a cosmic-ray-driven dynamo in dwarf galaxies. Astron Astrophys 562:A136

Soida M, Beck R, Urbanik M, Braine J (2002) Magnetic fields in the absence of spiral density waves-NGC 4414. Astron Astrophys 394:47

Soida M, Krause M, Dettmar R-J, Urbanik M (2011) The large scale magnetic field structure of the spiral galaxy NGC 5775. Astron Astrophys 531:A127

Sokoloff D, Shukurov A, Krause M (1992) Pattern recognition of the regular magnetic field in disks of spiral galaxies. Astron Astrophys 264:396

Sokoloff DD, Bykov AA, Shukurov A et al (1998) Depolarization and Faraday effects in galaxies. Mon Not R Astron Soc 299:189

Stenflo JO (2012) Basal magnetic flux and the local solar dynamo. Astron Astrophys 547:A93

Stepanov R, Arshakian TG, Beck R, Frick P, Krause M (2008) Magnetic field structures of galaxies derived from analysis of Faraday rotation measures, and perspectives for the SKA. Astron Astrophys 480:45

Stepanov R, Shukurov A, Fletcher A et al (2014) An observational test for correlations between cosmic rays and magnetic fields. Mon Not $R$ Astron Soc 437:2201

Stil JM, Krause M, Beck R, Taylor AR (2009) The integrated polarization of spiral galaxy disks. Astrophys J 693:1392 
Strassmeier KG, Ilyin IV (2009) The E-ELT: a chance to measure cosmic magnetic fields. In: Moorwood A (ed) Science with the VLT in the ELT era, p 255

Strong AW, Moskalenko IV, Reimer O (2000) Diffuse continuum gamma rays from the Galaxy. Astrophys J 537:763

Sun X-H, Reich W (2010) The Galactic halo magnetic field revisited. Res Astron Astrophys 10:1287

Sun XH, Reich W, Waelkens A, Enßlin TA (2008) Radio observational constraints on Galactic 3D-emission models. Astron Astrophys 477:573

Sun XH, Rudnick L, Akahori T et al (2015) Comparison of algorithms for determination of rotation measure and Faraday structure. I. 1100-1400 MHz. AJ 149:60

Tabatabaei FS, Beck R, Krügel E et al (2007) High-resolution radio continuum survey of M 33. II. Thermal and nonthermal emission. Astron Astrophys 475:133

Tabatabaei FS, Krause M, Fletcher A, Beck R (2008) High-resolution radio continuum survey of M 33 . III. Magnetic fields. Astron Astrophys 490:1005

Tabatabaei FS, Berkhuijsen EM, Frick P, Beck R, Schinnerer E (2013a) Multi-scale radio-infrared correlations in M 31 and M 33: the role of magnetic fields and star formation. Astron Astrophys 557:A129

Tabatabaei FS, Schinnerer E, Murphy EJ et al (2013b) A detailed study of the radio-FIR correlation in NGC 6946 with Herschel-PACS/SPIRE from KINGFISH. Astron Astrophys 552:A19

Tamburro D, Rix H-W, Leroy AK et al (2009) What is driving the HI velocity dispersion? AJ 137:4424

Tang Y-W, Ho PTP, Koch PM et al (2009) Evolution of magnetic fields in high-mass star formation: linking field geometry and collapse for the W51 e2/e8 cores. Astrophys J 700:251

Taylor AR, Stil JM, Sunstrum C (2009) A rotation measure image of the sky. Astrophys J 702:1230

Tingay SJ, Kaplan DL, McKinley B et al (2013) On the detection and tracking of space debris using the Murchison Widefield Array. I. Simulations and test observations demonstrate feasibility. AJ 146:103

Tüllmann R, Dettmar R-J, Soida M, Urbanik M, Rossa J (2000) The thermal and non-thermal gaseous halo of NGC 5775. Astron Astrophys 364:L36

Urbanik M, Elstner D, Beck R (1997) Observational signatures of helical galactic magnetic fields. Astron Astrophys 326:465

Van Eck CL, Brown JC, Stil JM et al (2011) Modeling the magnetic field in the Galactic disk using new rotation measure observations from the Very Large Array. Astrophys J 728:97

Van Eck CL, Brown JC, Shukurov A, Fletcher A (2015) Magnetic fields in a sample of nearby spiral galaxies. Astrophys J 799:35

van Haarlem MP, Wise MW, Gunst AW et al (2013) LOFAR: the Low-frequency Array. Astron Astrophys 556:A2

Vázquez-Semadeni E, Kim J, Ballesteros-Paredes J (2005) Star formation efficiency in driven, supercritical, turbulent clouds. Astrophys J Lett 630:L49

Völk HJ (1989) The correlation between radio and far-infrared emission for disk galaxies - a calorimeter theory. Astron Astrophys 218:67

Vollmer B, Soida M, Beck R et al (2007) The characteristic polarized radio continuum distribution of cluster spiral galaxies. Astron Astrophys 464:L37

Vollmer B, Soida M, Beck R et al (2013) Large-scale radio continuum properties of 19 Virgo cluster galaxies. The influence of tidal interactions, ram pressure stripping, and accreting gas envelopes. Astron Astrophys 553:A116

Wang P, Abel T (2009) Magnetohydrodynamic simulations of disk galaxy formation: the magnetization of the cold and warm medium. Astrophys J 696:96

Watson WD, Wiebe DS, Crutcher RM (2001) Irregular magnetic fields in interstellar clouds and variations in the observed circular polarization of spectral lines. Astrophys J 549:377

Weżgowiec M, Urbanik M, Vollmer B et al (2007) The magnetic fields of large Virgo cluster spirals. Astron Astrophys 471:93

Weżgowiec M, Urbanik M, Beck R, Chyży KT, Soida M (2012) The magnetic fields of large Virgo cluster spirals. II. Astron Astrophys 545:A69

Wiener J, Zweibel EG, Oh SP (2013) Cosmic ray heating of the warm ionized medium. Astrophys J 767:87

Yoast-Hull TM, Everett JE, Gallagher JS III, Zweibel EG (2013) Winds, clumps, and interacting cosmic rays in M82. Astrophys $\mathrm{J} 768: 53$

Yoast-Hull TM, Gallagher JS III, Zweibel EG, Everett JE (2014) Active galactic nuclei, neutrinos, and interacting cosmic rays in NGC 253 and NGC 1068. Astrophys J 780:137 